\title{
AN EXPLORATION OF REAL-TIME FUNCTIONAL MAGNETIC RESONANCE IMAGING NEUROFEEDBACK IN COGNITION
}

\author{
PhD Thesis \\ in partial fulfilment of the requirements \\ for the degree \\ “Dr. rer. nat." \\ in the Neuroscience Program \\ at the Georg August Universität Göttingen, \\ Faculty of Biology
}

\section{Submitted by \\ Wan Ilma Dewiputri}

Born in

Penang, Malaysia

Goettingen, 2014 



\title{
AN EXPLORATION OF REAL-TIME FUNCTIONAL MAGNETIC RESONANCE IMAGING NEUROFEEDBACK IN COGNITION
}

\author{
PhD Thesis \\ in partial fulfilment of the requirements \\ for the degree \\ "Dr. rer. nat." \\ in the Neuroscience Program \\ at the Georg August Universität Göttingen, \\ Faculty of Biology
}

\section{Submitted by \\ Wan Ilma Dewiputri}

Born in

Penang, Malaysia

Goettingen, 2014 
Doctoral thesis committee:

Prof. Dr. Jens Frahm

Biomedizinische NMR Forschungs

$\mathrm{GmbH}$

Max-Planck-Institut für biophysikalische

Chemie

Am Fassberg 11

37077 Göttingen

Prof. Dr. Stefan Treue

Cognitive Neuroscience Laboratory

Deutsches Primatenzentrum GmbH

Leibniz-Institut für Primatenforschung

Kellnerweg 4

37077 Göttingen

Prof. Dr. Michael Waldmann

Georg-Elias-Müller Institut für

Psychologie

Georg-August-Universität Göttingen

Abteilung 1

Goßlerstr.14

37073 Göttingen
Additional referees for thesis

defense:

PD Dr. Peter Dechent

MR-Forschung in der Neurologie und

Psychiatrie

Abteilung Kognitive Neurologie

Universitätsmedizin Göttingen

Robert-Koch-Str. 40

37075 Göttingen

Prof. Dr. Alexander Gail

Sensorimotor Group

Deutsches Primatenzentrum GmbH

Leibniz-Institut für Primatenforschung

Kellnerweg 4

37077 Göttingen

Prof. Dr. Hansjörg Scherberger

Neurobiology Laboratory

Deutsches Primatenzentrum GmbH

Leibniz-Institut für Primatenforschung

Kellnerweg 4

37077 Göttingen

Thesis Submission Date: 8 September 2014

Thesis Defense Date: 14 October 2014 
Herewith I declare that I have written this thesis independently and with no other sources and aids than quoted.

Göttingen, 8 September 2014

Wan Ilma Dewiputri 
To my family 


\section{Acknowledgements}

Many thanks to Prof. Dr. Jens Frahm for giving me a chance to pursue my interest in cognitive neuroscience at his department first for my lab rotation and then this doctoral project. Thank you for allowing me the freedom to explore my interest in neuroimaging, for the encouragement and support, and for providing excellent environment and facilities at the department.

I'd like to express sincere gratitude and heartfelt appreciation to my mentors and advisors, Dr. Renate Schweizer and Dr. Tibor Auer for all their guidance, encouragement, stimulating discussions, enthusiasm, and commitment in my development as a doctoral student. I thank them for not only being great role-models in science, but also for the friendship we fostered over these three years. I wouldn't have been able to do it without the support of these amazing individuals.

To my thesis committee members, Prof. Dr. Stefan Treue and Prof. Michael Waldmann, thank you for all the helpful suggestions and comments during the thesis committee meetings and for the help in getting the ethics approval.

I am very grateful to Dr. Tibor Auer and also Dr. Henry Luetcke for their technical and programming expertise in developing the specific stimulations and analysis programs that I used in this work. Tibor's dedication in sharing his expertise, and his patience in teaching me, were invaluable to my project. My thanks also go to Kurt Böhm for being very approachable and ever ready to help with general computing issues at the scanner console and at my work station.

At the Max Planck Institute, I'd like to thank my colleagues at the Biomed NMR for the positive environment in general, for the stimulating discussions and conversations, the stress-relieving soccer and badminton sessions among others. Special thanks to Dr. Meike Schweisfurth for her support and valuable insights as a senior doctoral student in the similar field and in the same PhD program, and also for the good company during the conferences. To Sylke Wallbrecht, I really appreciate her help in administrative matters, navigating through German bureaucracy would've been more intimidating without her help. To the librarians at Otto Hahn Bibliothek, I express gratitude for the help in getting the various literatures essential to my work. 
Thanks to Dr. Renate Schweizer, Dr. Tibor Auer, Dr. Sabine Hofer and Suhaidah Mohd Joffry for proofreading parts of this thesis and supporting me with critical and helpful comments.

Many thanks to Prof. Dr. Michael Hörner and Sandra Drube from the IMPRS Neuroscience program who have been very supportive and helpful even before I came to Germany. Their help made settling in Göttingen a smooth process.

Not to forget all of my subjects, who were not only dedicated to come for the multiple and intense scanning sessions, but also were enthusiastic about the study as well!

For stipends, financial assistance and grants throughout my PhD journey, I'd like to thank: Ministry of Education of Malaysia, Universiti Sains Malaysia, International Max Planck Research School, Göttingen Graduate School for Neurosciences, Biophysics, and Molecular Biosciences (GGNB), and The Bernstein Focus: Neurotechnology (BFNT).

To the colleagues at the Department of Neuroscience at Universiti Sains Malaysia, I'd like to thank Prof. Jafri Malin Abdullah for all his help, support and valuable insights about science in Malaysia; Aini Ismafairus Abdul Hamid for the stimulating discussions about neuroimaging and for the friendship.

To my friends in Germany who have been very supportive and understanding, thanks for the fun and memorable times which helped in making this PhD journey a happy and fulfilling one, and not to forget my fellow Adelaide alumni friends who inspired me in various ways.

My warmest thanks goes to my family: my husband Annas, for his love, understanding and constant encouragement, my mom for giving me strength to persevere, my late dad for inspiring my thirst for knowledge, my brothers for their support from the distance, and my in-laws for their understanding and kindness. Terima kasih.

Last but not least, I thank God for His grace bestowed upon me. Alhamdulillah. 


\section{Contents}

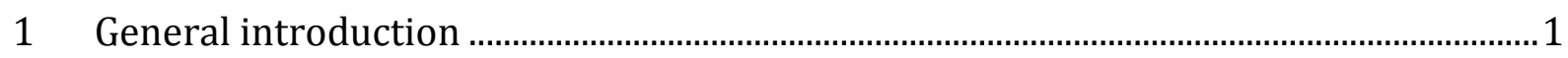

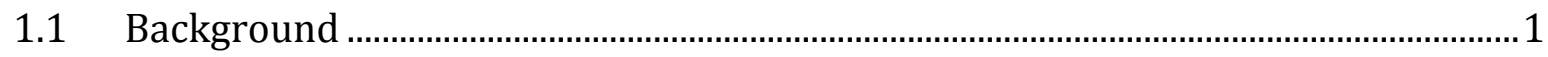

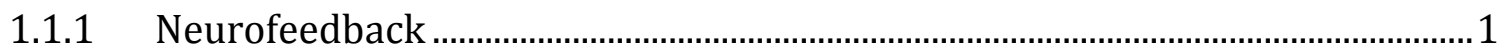

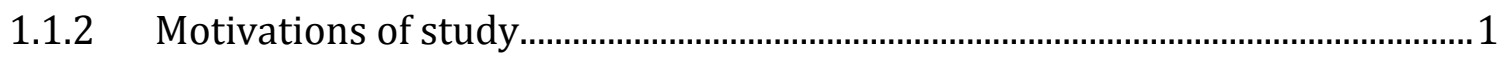

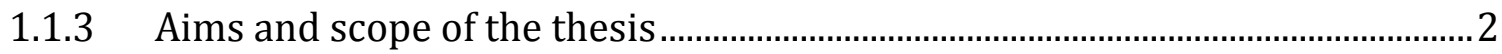

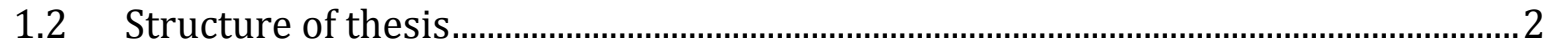

1.3 Functional MRI in neurofeedback: implementations and applications ....................3

2 Functional localization of the anterior mid-cingulate cortex for real-time fMRI

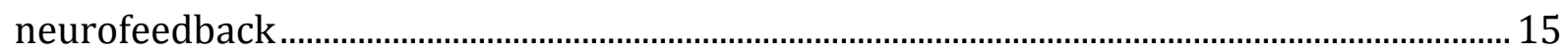

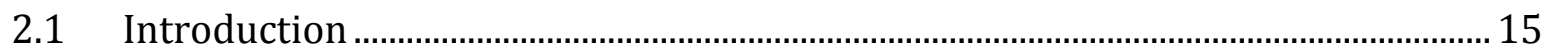

2.1.1 The many facets of the anterior mid-cingulate cortex....................................... 15

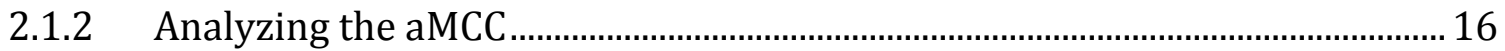

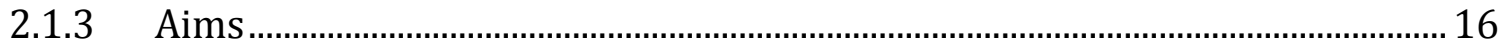

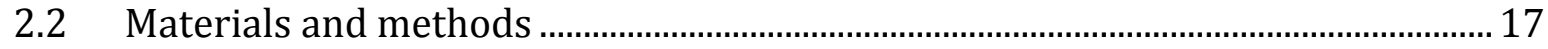

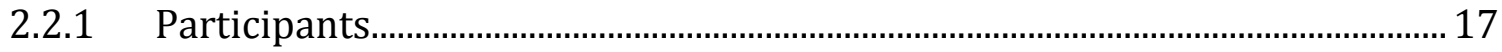

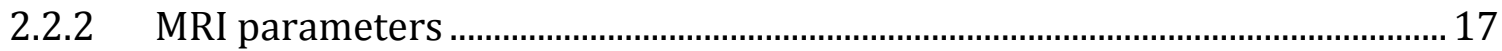

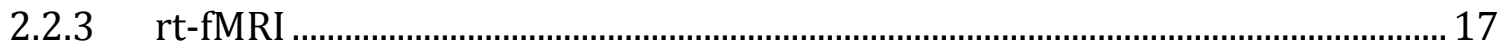

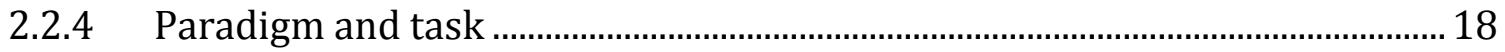

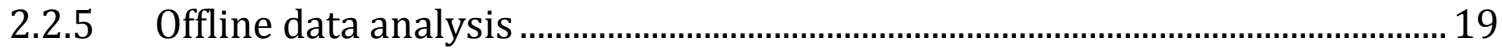

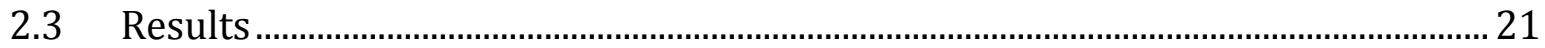

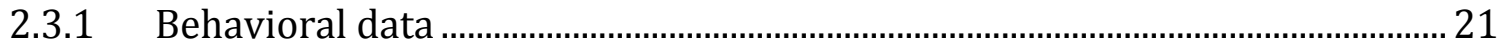

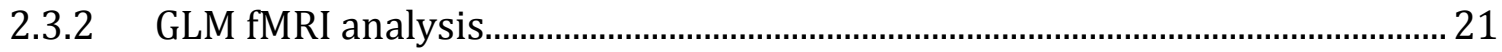

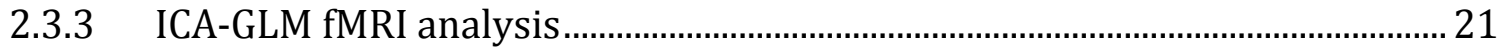

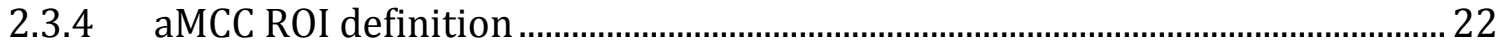

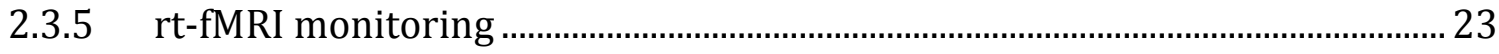

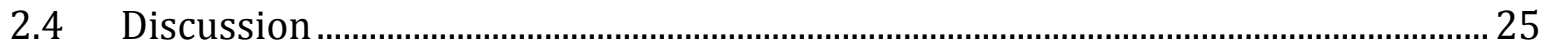


2.4.1 GLM and ICA-GLM analysis of the functional localizer task 25

2.4.2 Activation of aMCC as part of a cognitive network ...............................................26

2.4.3 Functional localizer task guided by rt-fMRI.......................................................... 26

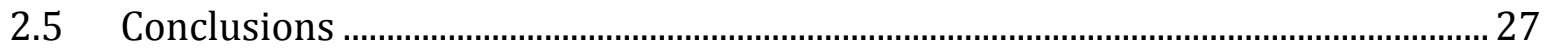

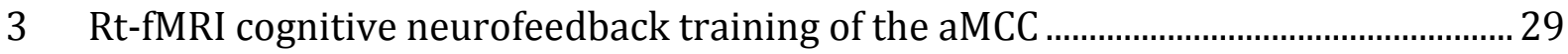

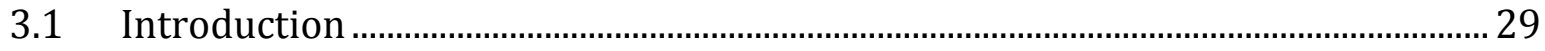

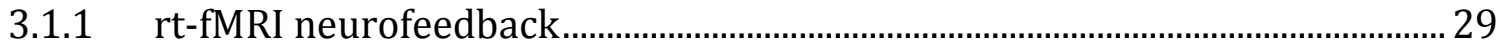

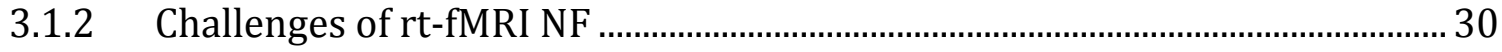

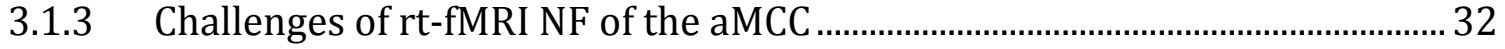

3.1.4 Development of a novel rt-fMRI paradigm: the Serial paradigm..................... 32

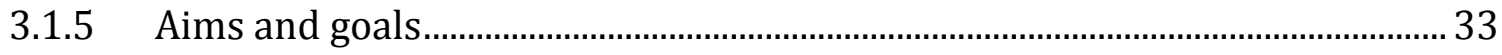

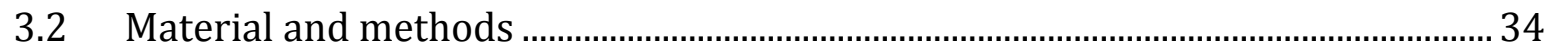

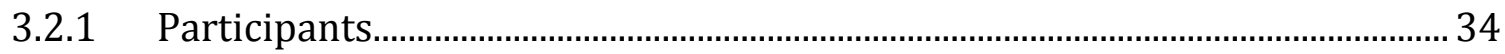

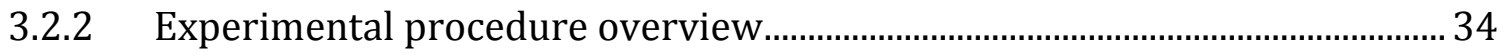

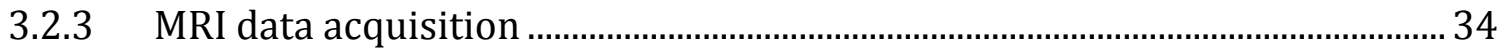

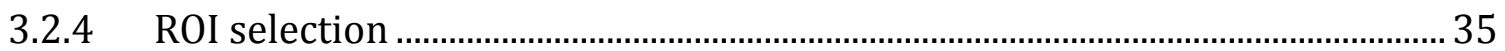

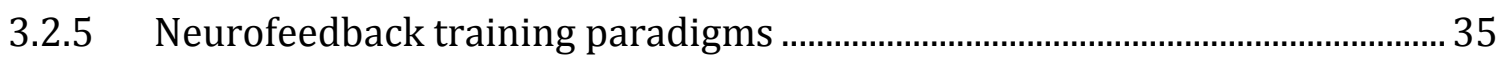

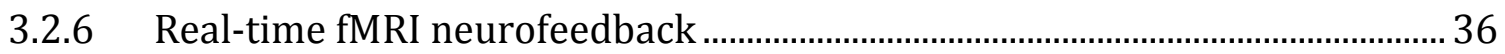

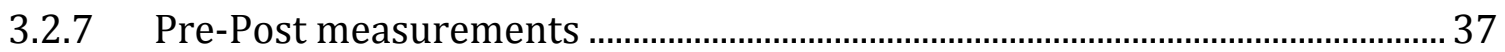

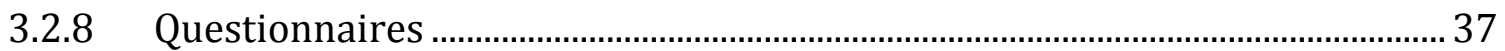

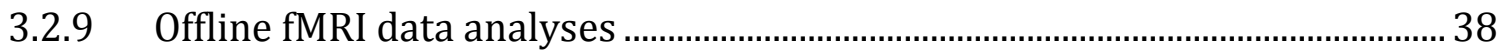

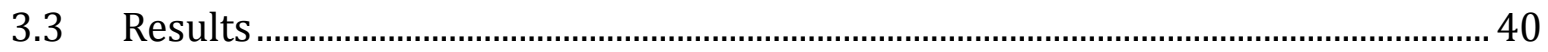

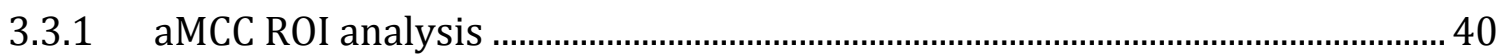

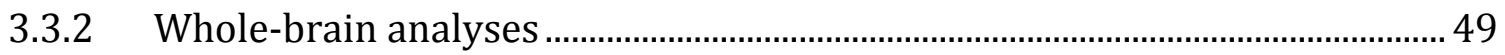

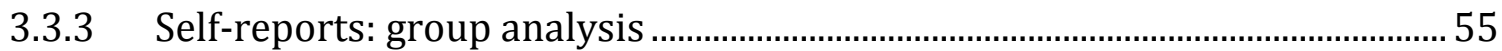

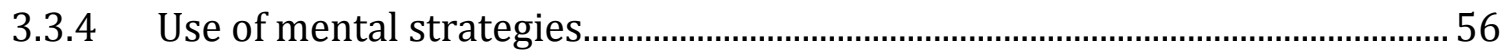

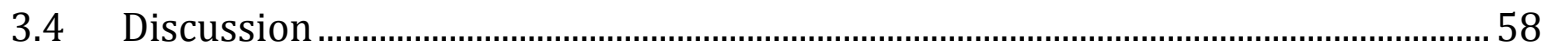




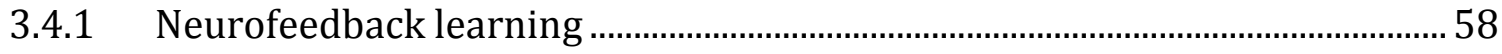

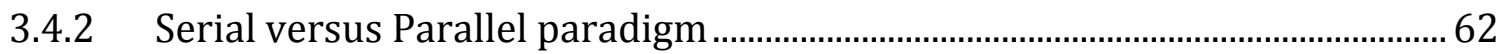

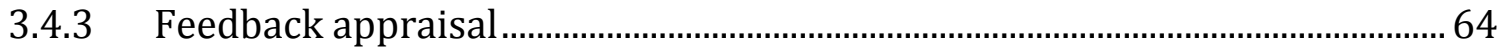

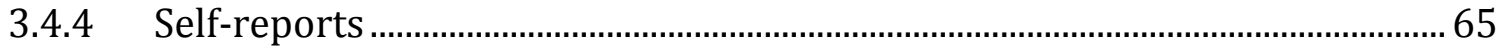

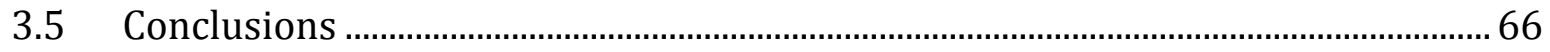

$4 \quad$ Generalization of neurofeedback training to behavior ....................................................... 69

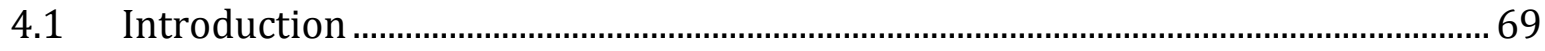

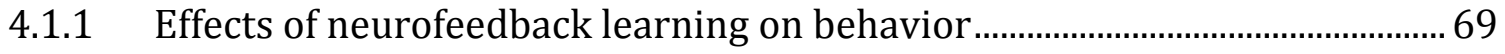

4.1.2 CPT

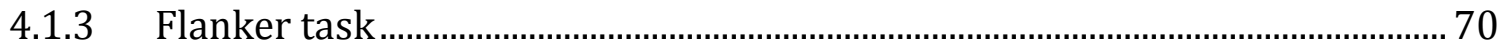

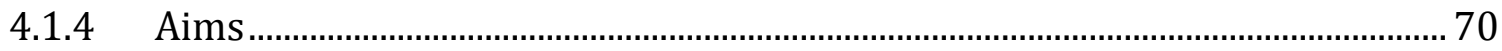

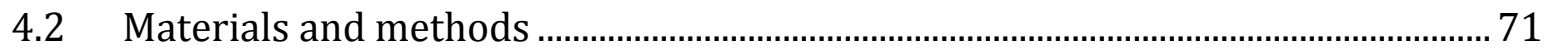

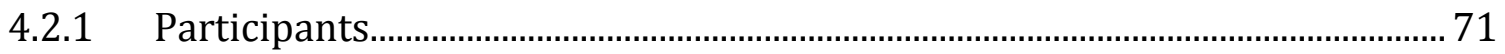

4.2.2 Experimental procedure overview...................................................................... 71

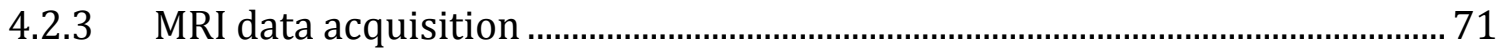

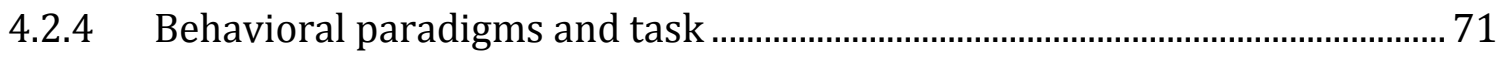

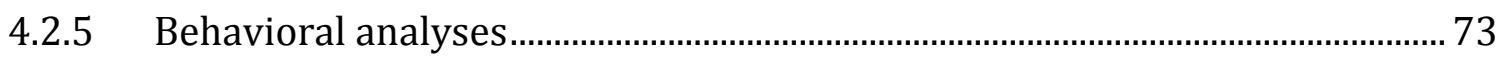

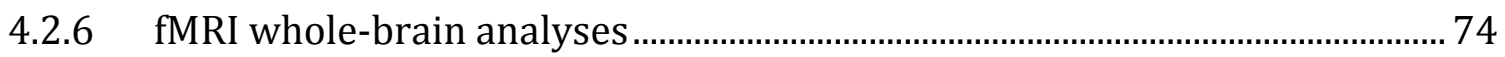

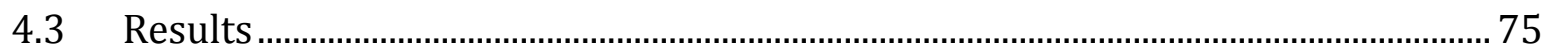

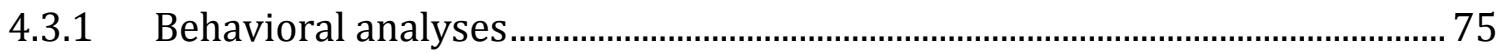

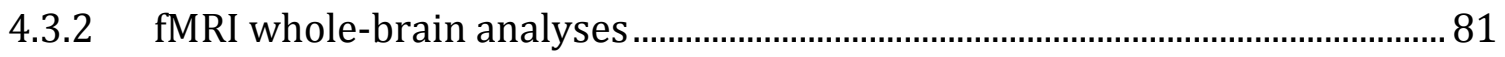

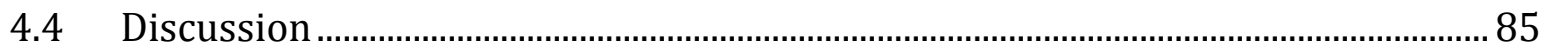

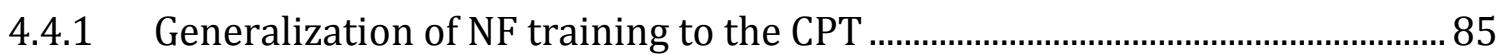

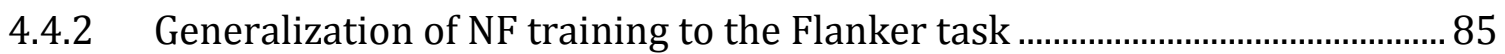

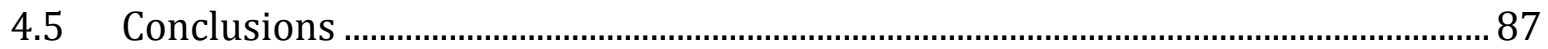

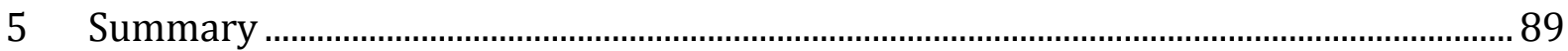

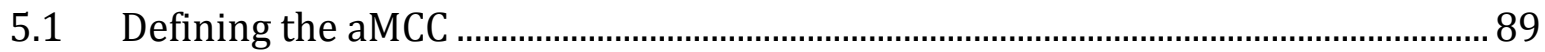


5.2 Neurofeedback training of the aMCC

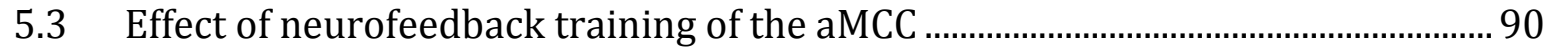

$6 \quad$ Outlook

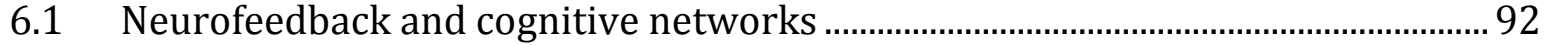

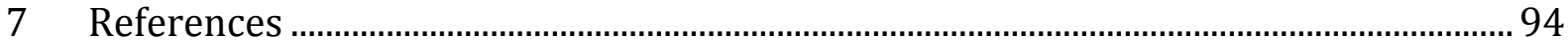

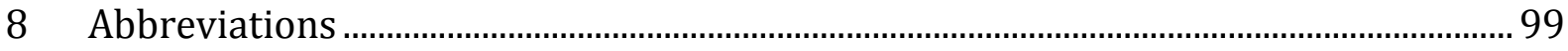

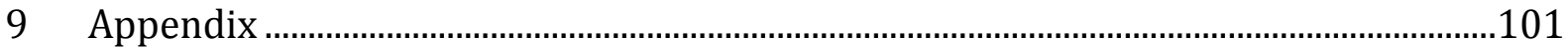

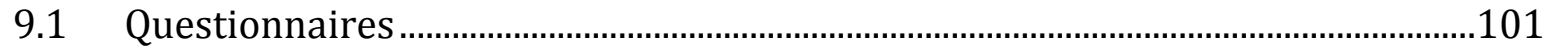

9.1.1 Example of a pre-session questionnaire ............................................................ 101

9.1.2 Example of a post-session questionnaire for Serial paradigm .......................103

9.1.3 Example of a post-session questionnaire for Parallel paradigm ...................107

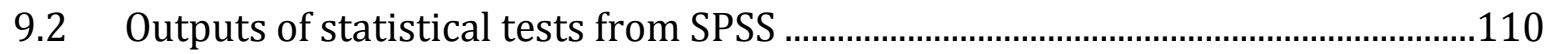

9.2.1 Rt-fMRI cognitive neurofeedback training of the aMCC..................................110

9.2.2 Generalization of neurofeedback training to behavior ...................................118

Curriculum Vitae 



\section{General introduction}

\subsection{Background}

\subsubsection{Neurofeedback}

eurofeedback (NF) is a variation of biofeedback that facilitates learned self-
regulation of an individual's own brain activity, with the goal to produce
changes in brain function or behavior. Feedback on brain activity can be provided through real-time functional magnetic resonance imaging (rt-fMRI) which measures the blood-oxygenation level-dependent (BOLD) signal representing neural activity. NF training enables subject to learn how to influence a target brain area that is not normally under voluntary control, by usage of mental strategies that engage the target brain area, such as mental imagery. Subjects get feedback of their brain activity from the target area, which is presented in the form that is easily interpretable to inform subjects about their performance. By viewing and appraising the feedback, an individual can potentially learn to self-regulate their own brain activity. The learning process is viewed as operant learning where an individual's behavior is modified by its consequences. In NF, this means adapting the mental strategies by trial-and-error based on the antecedent feedback.

\subsubsection{Motivations of study}

The motivation of the current thesis stems from a preceding study in our lab on rt-fMRI NF of the somatomotor cortex (SMC) (Auer and Frahm, 2011). The present work aims to conceptionally extend previous findings by moving on to explore rt-fMRI NF in a circumscribed cognitive brain area. The cognitive brain area of interest is the anterior mid-cingulate cortex (aMCC), a division of the cingulate cortex that has a central role in cognition - integrating cognitive, limbic and motor control (Shackman et al., 2011). RtfMRI NF studies on the cingulate cortex are scarce, because of the need to meet several new challenges. The first difficulty arises from the technical and physiological nature of the BOLD signal, which manifests in a feedback delay of $\sim 10$ seconds. This in turn, brings about a psychological challenge to the conventional rt-fMRI paradigm; specifically, the implicit temporal contiguity of the delayed feedback signal and the higher cognitive load induced by simultaneously performing a mental strategy and appraising the feedback. Secondly, rt-fMRI NF on the aMCC is also confronted with the problem of self-regulating the selected brain area itself, both in terms of lack of clear 
mental strategies to voluntarily activate the aMCC, and possible confounds in regulating a brain region that itself is involved in feedback appraisal. All of these challenges are described in more detail in this thesis. The work presented here is the first extensive rtfMRI NF study of the aMCC.

\subsubsection{Aims and scope of the thesis}

In the experimental framework of a rt-fMRI NF study, the initial step is to define a target region to be trained. Thus, the first goal of this thesis (Chapter 2) was to establish a robust functional localization method to define the aMCC. One behavioral test, the Continuous Performance Task (CPT) is employed; and two different fMRI analysis methods are compared, i.e. the General Linear Model (GLM) and Independent Component Analysis incorporating GLM (ICA-GLM).

The next step is to train the target region with NF, so that the second goal (Chapter 3) was to examine the possibility of learned self-regulation of the aMCC through extensive rt-fMRI training. For this purpose, a novel NF paradigm is introduced that uncouples self-regulation and feedback appraisal to resolve the challenges involved in rt-fMRI NF of the aMCC. This novel "Serial" paradigm is compared with the conventional "Parallel" NF paradigm. In addition, control groups which do not undergo NF training are included. This chapter also explores factors that promote learning in self-regulation of the aMCC.

The final step is to test the behavioral effects of rt-fMRI NF training. This third goal (Chapter 4) specifically asks if successful NF self-regulation of the aMCC leads to changes in behavioral measures and/or brain activity. Two behavioral tests, the CPT and the Flanker task, were employed before and after rt-fMRI NF training to measure such changes.

\subsection{Structure of thesis}

This thesis is divided into five chapters. Chapter 1 is a general introduction to NF. Three main chapters (Chapter 2, 3, and 4) contain the bulk of the experimental studies using rt-fMRI NF. Each of these chapters has its own Introduction, Material and Methods, Results and Discussion section. For a more specific and in-depth introduction, the reader is kindly referred to the individual chapters. Chapter 5 offers a summary, which collectively reviews the work in this thesis. Chapter 6 provides an outlook providing additional thoughts about future prospects of rt-fMRI NF aMCC study. 


\subsection{Functional MRI in neurofeedback: implementations and applications}

This part is presented as published in the peer-reviewed journal Malaysian Journal of Medical Sciences. This manuscript describes methodological aspects of functional magnetic resonance imaging (fMRI) and real-time fMRI (rt-fMRI), implementations of rtfMRI NF, a short overview of NF training in various brain regions, and possible applications of NF as a supplementary therapy tool. Permission from the Malaysian Journal of Medical Sciences has been obtained for the use of this publication as part of a doctoral thesis. 


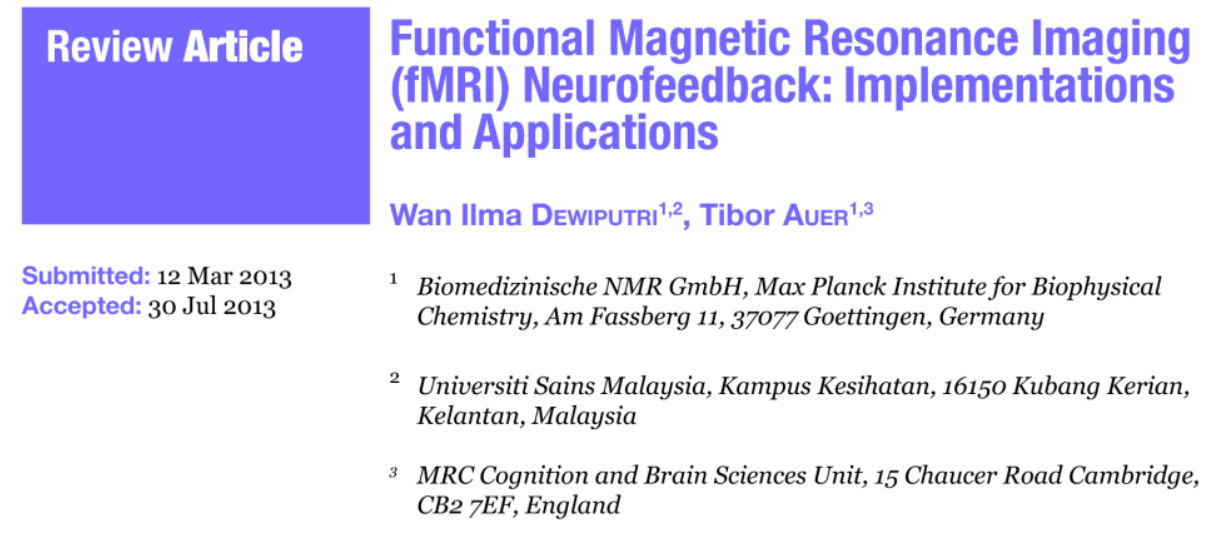

Abstract

Neurofeedback (NFB) allows subjects to learn how to volitionally influence the neuronal activation in the brain by employing real-time neural activity as feedback. NFB has already been performed with electroencephalography (EEG) since the 1970s. Functional MRI (fMRI), offering a higher spatial resolution, has further increased the spatial specificity. In this paper, we briefly outline the general principles behind NFB, the implementation of fMRI-NFB studies, the feasibility of fMRI-NFB, and the application of NFB as a supplementary therapy tool.

Keywords: fMRI, learning, neurofeedback, self-regulation

\section{Neurofeedback Using Functional Magnetic Resonance Imaging}

Neurofeedback (NFB) is a technique which allows subjects to learn how to volitionally influence the neuronal activation in the brain. The principle behind NFB training in general is that brain activity is self-modifiable through operant conditioning where the subjects are provided with feedback about ongoing neuronal activation with the goal to regulate it, and a "reward" is given should a certain level of activity be achieved. This volitional control of defined aspects of the central nervous system was successfully implemented at first using electroencephalography (EEG), whereby healthy subjects learn to control their electrical brain activity. EEG-NFB has been effectively applied to treat clinical conditions such as attention deficit hyperactivity disorder (ADHD) $(1,2)$ and epilepsy $(3,4)$.

The concept of "interactive experimental paradigms' was envisioned nearly two decades ago with the advent of real-time functional magnetic resonance imaging (rt-fMRI) (5). Rt-fMRI, which allows for high spatial resolution (in the range of millimetres) and imaging of activity across the entire brain within a couple of seconds, all done non-invasively without the need of surgery or injection of contrast agents, has paved the way for a new NFB paradigm. fMRI measures the blood oxygenation level-dependent (BOLD) response, i.e., signal differences due to local changes in the concentration of deoxygenated haemoglobin in the brain tissue, which depends on neuronal metabolism and activity. Specifically, the BOLD contrast is a result of magnetic field inhomogeneity change brought about by varying levels of deoxyhaemoglobin $(\mathrm{dHb})$ in the intracellular space of the red blood cells in blood vessels. $\mathrm{dHb}$ distorts the magnetic field around the blood vessel, enhancing the magnetic field inhomogeneity and thus, lowering the BOLD signal. Yet neural excitation increases the BOLD signal. This is due to a regional increase in cerebral blood flow (CBF) that exceeds the oxygen consumption rate, which ultimately results in an oversupply of oxygenated blood. Thus, the net effect is a drop in $\mathrm{dHb}$ concentration, which leads to an increase in the signal strength (as reviewed in $(6,7)$ ). The maximal change of the BOLD signal in response to neural activity is delayed by approximately 6 seconds. The relationship between the measured fMRI signal and the underlying neural activity has been thoroughly investigated, and there is solid evidence for a strong correlation between 
the BOLD signal and the local field potential as a measure of the electrical brain activity (8).

Recent technical developments have made it possible to analyse the data online as they are collected (hence, the term "real-time fMRI"), so that the resulting information is immediately available and can thus be used to guide a person's attempt at self-regulation according to the experimenter's parameters (9). NFB provides a new approach in neuroscience by studying brain plasticity and functional reorganization after continuous training of volitional control of defined brain regions (10).

In this paper, we focus on general principles behind NFB, and how it can be implemented using fMRI. With evidence from our results as well as from the literature, we demonstrate the feasibility of fMRI-NFB. Finally, we highlight some possible applications of NFB as a supplementary therapy tool.

How neurofeedback can influence brain functions

\section{Self-regulation of neural activity}

Numerous studies have proved that subjects can learn how to influence their brain activity. The growing interest is reflected in the increasing rate of publications on rt-fMRI from ca. one paper per year to about seven papers per year following the first publications on fMRI-NFB in 2002/2003 (11). Over the course of several NFB training sessions, subjects have successfully come up with a strategy or optimised an already existing one to elicit the desired level of activation. The importance of feedback from the ongoing activation as a predictor of success is undeniable and can be elucidated by various controls used in NFB experiments. The first control for the specificity of the NFB training effect is the transfer experiment, which is the comparison between preand post-training measurements, during which subjects try to regulate their own brain activity while not provided feedback. This comparison would tell us whether the subjects' ability to regulate their brain activity has improved. On a higher level, behavioural tests may prove whether the training effects generalise to produce any behavioural changes. The transfer experiments and the behavioural tests by themselves, only prove that the investigation had an effect on the targeted brain function (specificity in brain function). On the next level, control subjects who do not undergo NFB training can prove that the effect occurred due to the NFB training and not by chance (specificity in time). However, only subjects exposed to non-specific stimuli can prove that the effect is very specific to the NFB setup and not to non-specific elements of the training such as physical rest, scanner noise, concentration on the task, and observing a variable signal. These non-specific elements can be controlled with nofeedback training, sham feedback (feedback from a different region) or yoke feedback (feedback from another participant). Carefully designed studies must include one or more controls. A recent review by a group of prominent authors in the field of fMRI-NFB highlighted the importance of positive controls for future research (12), which would allow comparisons of NFB results to other means that efficiently achieve the desired effect.

The implications of learned self-regulation of a brain area are two-fold. The first implication of NFB would be to complement conventional neuroimaging methods in making inferences about brain function. Conventional neuroimaging experiments measure brain activity as the dependent variable which changes due to sensory stimulation or performing a behavioural task, while NFB allows investigating the effects of changing the BOLD signal (independent variable) on behaviour (dependent variable). Therefore, while conventional neuroimaging studies provide correlational information, fMRINFB complements these methods by additionally allowing researchers to investigate questions of causality (12). The potential impact would be substantial as corresponding capabilities are currently limited to interventional techniques such as transcranial magnetic stimulation, deep brain stimulation, and focal lesions. The second implication of the causal link between brain activity and behaviour is the possibility to modulate behaviour by influencing brain activity. One may argue against the success of NFB by saying that it merely trains the regulation of blood flow instead of the neuronal activity, particularly because biofeedback itself has been employed for modulating blood pressure (13). The modulation of the behaviour as a result of self-regulation of the corresponding neural activity is a proof against this argument.

\section{Change in brain function and structure}

In a study of London taxi drivers' brains structure, a correlation between the changes in volume of the right posterior hippocampus - a structure that stores spatial representations of the environment - and the time spent as a taxi driver has been reported, suggesting a plastic change in healthy adult brains in response to environmental demands (14). A follow-up study 


\section{General introduction}

Review Article | fMRI neurofeedback implementations and applications

detected no correlation of de novo navigational skills with brain structure; thus, providing further evidence that the structural changes observed in the taxi drivers were acquired and not merely due to innately good navigation skills (15). Recently, similar experience-dependent structural changes have been demonstrated in white matter, as well: a localised increase in fractional anisotropy, a measure of microstructure, has been detected in white matter underlying the intraparietal sulcus - an area involved in visually guided movements of the eye, hand, and visuo-motor coordinate transformation - following training of a complex visuo-motor skill (16). All these results provide evidence that not only the function but also the structure of our brain can be altered by regular mental training even in adulthood.

These promising results allow us to hypothesise that NFB, by its nature of subjecting participants to self-regulation training for a certain duration, may also induce structural brain changes. So far, studies have focused mainly on the feasibility of fMRI-NFB in healthy subjects or a clinical population, i.e. whether one can learn how to influence their own brain activity. Changes in the brain due to NFB training have been investigated only recently. Modulation of the relevant functional connectivity has been demonstrated after NFB training of the anterior insula $(17,18)$, the left amygdala (19), auditory attention-related brain areas (20) and the somatomotor cortex $(21,22)$. Modulation of functional connectivity has also been demonstrated in our study where four weeks of NFB training of the somatomotor cortex (i.e. target brain area) induced an increase in functional connectivity (23) between the target brain area and the anterior mid-cingulate cortex as a result of the increased cognitive control over the target brain area. Structural connectivity changes, however, proved to be ambiguous (24).

\section{A Typical fMRI Neurofeedback Experiment}

Data acquisition in rt-fMRI

Recent advances have rendered real-time analyses possible, so image reconstruction, transfer, and analysis can be accomplished within the time frame necessary for the acquisition of a single volumetric dataset (repetition time: TR) - typically within 2 seconds. Most scanners are equipped with a BOLD echo-planar-imaging (EPI) sequence enabling fMRI, but acquired volumes are usually available only in one package after finishing the whole measurement. Because built-in solutions for fMRI analysis are usually inadequate due to a lack of processing options, access to the volumes by an auxiliary tool which performs online fMRI analysis is also essential. Most companies provide option for real-time export of the acquired data which reconstructs each volume one-by-one right after its acquisition (i.e. online), therefore allowing parallel analysis with the acquisition. At the time of writing, some companies' solutions (e.g. Philips') work outof-the-box, while others (e.g. Siemens') require additional adjustments to ensure maximal temporal consistency (25).

Apart from the real-time access to the data, a separate computer dedicated to real-time analysis is also necessary. The computational burden of the real-time analysis is higher than that of any other NFB component, therefore this computer should be powerful enough to perform all the necessary processing. In other words, a computer with high processing power would allow a higher number of more complex processing steps. The results of the analysis need to be presented to the subject in parallel with the acquisition, which requires a stimulus-generating computer presenting the feedback to the subject through a beamer or LCDgoggles in the case of a visual feedback, or through headphones in the case of auditory feedback. A connection between the scanner and the stimulus-generating computer may be also useful (but not essential) to compensate for the temporal variability of the analysis and to synchronise feedback presentation with the acquisition via a trigger pulse from the scanner (Figure 1). Because this variability is usually well below the TR that is generally used, simply presenting the results of the analysis as soon as they are available can be also a viable solution.

Visual feedback is the most commonly used feedback modality because vision is the most dominant human sense, and visual feedback has been shown to surpass auditory feedback (26). The graphical representation of visual feedback varies from a continuously updated graph (27), smiling avatars (28) to fluctuating levels of a thermometer (29) or fire (30). The subjects are usually informed of the feedback presentation delay, which depends on the image acquisition and processing time, and the inherent delay of the BOLD signal, which takes 6 seconds to peak. This does not allow "immediate" feedback and control of the NFB signal like EEG-NFB does, thus leading to reduced contingency, which in some cases could render the NFB training with fMRI more difficult. 


\section{Data analysis}

There are a tremendous number of preprocessing techniques (motion correction to ensure spatial consistency in time; correction of the distortion due to magnetic field inhomogeneity; slice timing to ensure temporal consistency across slices acquired at slightly different time points; spatial normalisation to ensure spatial consistency across subjects; spatial filtering to improve signal-to-noise ratio at the expense of effective spatial resolution) and evaluation methods (univariate and multivariate; model-based and model-free approaches) at hand, and all of them are applicable in real time as well. The main challenge is how to increase speed while maintaining quality at the same time. Motion correction is the most important step, because it ensures the spatial consistency over time. It is also the most time-consuming; therefore, its optimisation usually means finding the trade-off between time and quality $(31,32)$. Distortion correction and spatial filtering may further improve the data quality (33).

The other essential step is to find the spatial correspondence between the volumes and the target region(s) of interest (ROI) selected for feedback. These ROIs can be selected on a functional or anatomical basis, or based on an atlas. Using a functional localiser only requires a withinsubject (i.e. rigid-body) registration, which can be easily combined with the motion correction: we only have to specify the first scan of the functional localiser measurement as a reference scan for the motion correction. Using the anatomical scan of the given subject or an atlas additionally requires affine registration and involves spatial filtering. The former is more computationally intensive, while the latter decreases effective spatial resolution. A more preferable approach could be to map the ROI obtained from the anatomy or an atlas to the functional volume of the actual measurement (34), which is fast and preserves the original functional data.

There are two main groups of evaluation approaches to analyse fMRI data in real time. The growing-window or incremental approach (35) simply uses all available data; therefore, its statistical power increases with more acquired data. On the other hand, it rather provides information about the mean activation over time. The sliding-window or constant approach uses only a temporal subset of data, thus keeping the

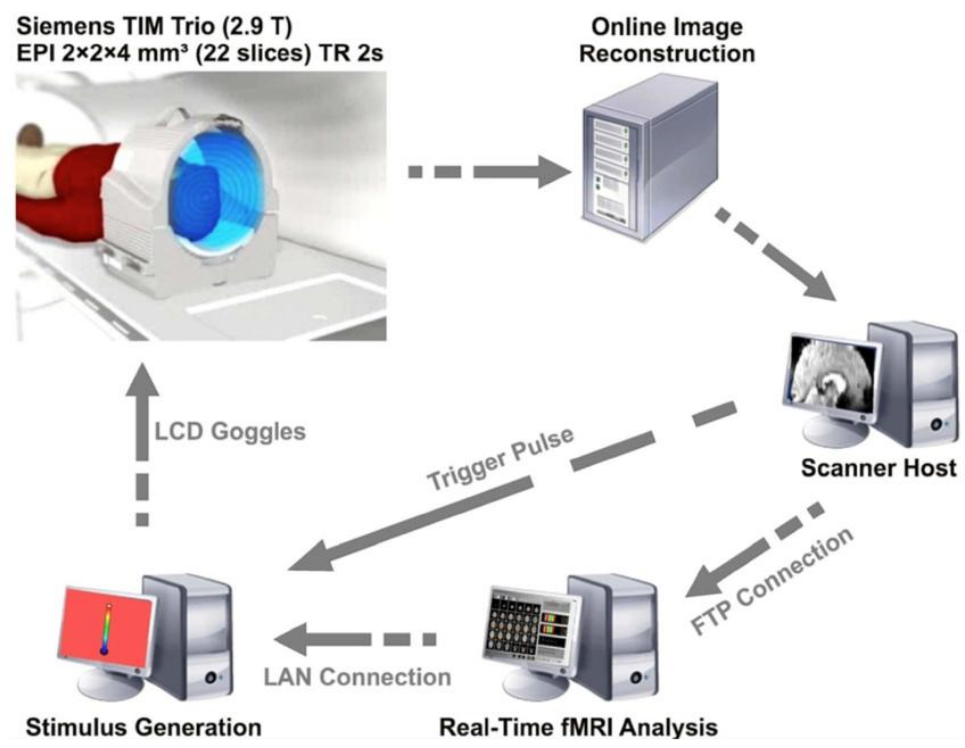

Figure 1: Real-time fMRI neurofeedback setup. The real-time fMRI neurofeedback system set up is a closed loop. The subject tries to self-regulate their brain activity while getting feedback of their own brain activity from the stimulus-generating computer through a beamer or LCD-goggles. 


\section{General introduction}

Review Article | fMRI neurofeedback implementations and applications

statistical power fixed and reduced compared with the maximum achievable with the growingwindow approach. The sliding-window approach (36) reflects the current activation state better, and the smaller the window's width, the better the temporal resolution. For both approaches, all the statistical evaluation techniques developed for conventional fMRI are available, such as $t$ tests (37), correlation analysis (38), (multiple) regression (39), and independent component analysis (40). The scan-to-scan approach can be considered as a sliding-window approach with a window width of one single scan; therefore it provides maximum temporal resolution. However, this approach suffers the most from the signal fluctuations resulting from both technical and physiological noise. The loss of temporal information about the noise can be partially compensated for in the spatial domain; namely by accumulating even weak information available in more voxels. The most straightforward and the most widely employed approach is to average the signal of a subset of voxels (ROI-based analysis). In this case, a "background" ROI taken from a non-involved area should also be used to correct for the global changes during the measurement due to changes in the general arousal state or in the breathing rate. The introduction of "control" phases during the measurement allows taking the last several time points of the previous control period as reference, thus correcting for the signal drift:

$$
N S_{t}=\left(S_{t} / S_{\text {previous_control }}-1\right) \times 100
$$

where $N S_{t}$ and $S_{t}$ correspond to the normalised and raw signal intensity at time point $t$, respectively, while $S_{\text {previous control }}$ refers to the mean signal intensity during the previous control period. To increase the robustness and minimise the sensitivity of the normalised signal to signal fluctuations around zero, a double logistic-like function $(f)$ for calculating the feedback signal (FS) with the following characteristics can be implemented: a relatively flat centre between -0.5 and 0.5 NS ensures that small changes in NS will have limited effect on $F S$, while plateaus at $-2 N S$ and $2 N S$ control saturation (Figure 2). These values may be adjusted for the actual experiment:

$$
F S=f\left(N S_{t}\right)
$$

A more sophisticated approach for combining signals from more voxels is employing a pattern recognition technique, which offers good sensitivity even if the signals in each voxel of the ROI do not change identically (41). Its realtime implementation has the ability to provide feedback based on intuitive translations of "brain state" rather than localised fluctuations (42).

\section{Outline of a neurofeedback training}

NFB training enables the self-regulation of the activity of a defined brain region. In the case of fMRI-NFB, a region or network of interest may be selected from which the signal is then acquired. The selection of an ROI can be done structurally as well as functionally. In structural localisation, ROIs are generally defined based on macroanatomy, such as gyral anatomy. It is best to define such ROIs for each individual based on their own anatomy, since there can be substantial inter-subject variability. In functional localisation, a separate 'localiser' measurement is used to identify voxels in a particular anatomical region that show a particular response (e.g. voxels in the fusiform gyrus that are more responsive to faces than to other objects); these voxels are then explored to examine their response to NFB (43).

Then subjects undergo several training sessions in which they are instructed to come up with their own mental strategy and optimise it in order to enhance self-regulation of their brain activity. The mental strategy is achieved by mental imagery, where the subjects need to imagine situations or feelings without overtly experiencing them. Concurrently, subjects also view feedback originating from their own brain activity, which guides them towards selecting the most efficient strategy. It is hoped that during successful NFB training, the subjects' ability to

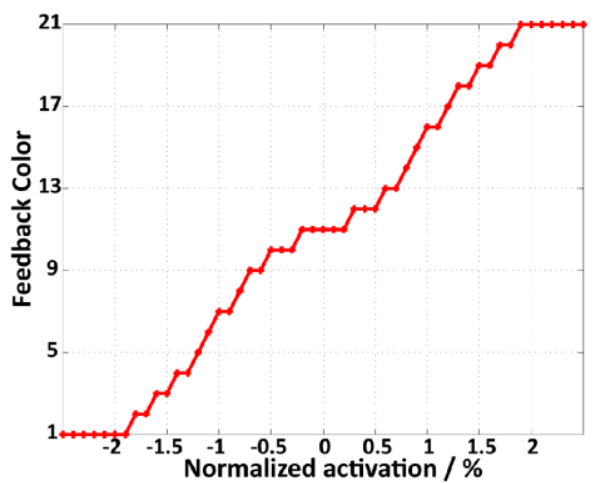

Figure 2: Double logistic-like function used to calculate feedback signal (FS). 
influence their brain activity would gradually increase, and training effects would be manifested when subjects find a strategy that reliably elicits the desired brain activation(s) even without the help of feedback. Therefore, the ultimate test of the NFB training effect is the transfer condition, which is the comparison between pre- and posttraining measurements, during which subjects try to regulate their own brain activity while not provided feedback. This comparison would tell us how much the subjects' ability to regulate their brain activity has improved. In addition, behavioural tests may also be performed preand post-NFB training to investigate whether the training effects generalise to produce any behavioural changes.

\section{Neurofeedback Training in Various Brain Regions}

Ever since fMRI-NFB has been shown to be feasible, it has paved the way for researchers to experiment with fMRI-NFB training in various areas of the brain. Researchers have trained subjects to volitionally control specific cortical and subcortical areas such as the primary motor cortex (22), the supplementary motor area (SMA) (27), the anterior cingulate cortex (ACC) $(27,30)$, the amygdala $(19,44)$, and the insula (45). Here, we attempt to briefly summarise major studies of fMRI-NFB training in these areas.

\section{Somatomotion: Training the somatomotor} cortex

The somatomotor cortex (SMC) is responsible for planning, control, and execution of voluntary movements. The SMC is easily accessible and produces a robust signal; moreover, it is a "natural" target for a brain-computer interface, an approach attempting to establish mind control over machines such as computers, robots, and prosthetics. Therefore, the SMC was one of the first (46) and perhaps the most often targeted areas when investigating the feasibility of fMRINFB.

In a pioneering study, subjects were shown to be able to increase their BOLD signal activity in the hand area of the SMC more significantly than the controls who did not receive valid feedback (21). This also held true when two weeks of NFB training included only daily self-practice (without scanning) of motor-hand imagery established during an initial fMRI-NFB session (22). This group also showed that the NFB training resulted in the recruitment of additional neural circuitries implicated in motor skill learning such as the hippocampus and the limbo-thalamo-cortical pathway.

In the SMC, the success of NFB training possibly depends on the length and/or the distribution of training. Recent studies have shown that short training periods (1-4 runs within one day) $(47,48)$ could be inadequate to achieve self-regulation of an ROI. Our own results have shown that in an intensive 48-run NFB training period that spanned four weeks, subjects who underwent NFB training of the SMC showed a distinct increase of the BOLD signal in the SMC (49). In addition, successful NFB training of the SMC not only resulted in enhanced fMRI activation during transfer (i.e. without feedback) but also during overt finger movements (23). The latter also implies functional plasticity changes in the SMC in an overt movement condition involving activation of the trained neuronal substrate. We could also prove that training efficiency (i.e. how subjects were performing during the training) was highly correlated to the overall training success (pre- to post-training transfer), which helps us to predict the effect of training on the fly (50).

\section{Emotion: Training a single ROI and a whole} network

Emotions shape human beings' interaction with the world. Ever since the scientific community developed interest in understanding the neural basis of emotion and its relationship to cognitive function and behaviour, fMRI has been used as a tool to achieve this goal. As neuroimaging technology advanced, neuroscientists began to wonder if emotions can be self-regulated via NFB. One of the early fMRI-NFB studies in the domain of emotion was on the amygdala (51).

Since then, researchers have embarked on investigating the feasibility of fMRI-NFB in the emotional domain. Self-regulation has been investigated in the subgenual ACC (sACC) - a region involved in the generation of affective states and implicated in psychopathology $(52,53)$. In that study, subjects had to come up with one strategy for increasing positive mood (hence decreasing the SACC activity) during the NFB training. Subjects who had received feedback were able to decrease their sACC activity, in contrast to subjects who received sham feedback. Similarly, self-regulation of the amygdala using positive autobiographical memory retrieval was successful in subjects who received feedback but unsuccessful in the sham control group (19).

Training of areas involved in emotional regulation could be beneficial for patients who suffer from emotion impairment, which manifests 
Review Article | fMRI neurofeedback implementations and applications

in depression, bipolar disorder, and obsessivecompulsive disorder, among others. The aim of doing NFB training in these areas is to the reduce emotional symptoms of a particular disorder.

NFB using fMRI can be applied to train not only a single ROI but also a whole network. Training the emotion network in the brain, which includes the amygdala and the insula, has shown that subjects were able to modulate the activity of that network $(29,44)$. Training also generalised to a behavioural measure in which participants' sensitivity to aversive pictures had increased with learned regulation of the insula (29). Consequently, promising results in this domain have steered researchers towards using actual patients in NFB studies.

\section{Training the cognitive engine-Anterior Mid- Cingulate Cortex}

In addition to training the motor and limbic cortical areas that directly control action and emotion respectively, training the anterior midcingulate cortex (aMCC), the cognitive engine that drives both domains, has also been in the focus since the beginning (54). The aMCC is best described as a limbic premotor cortex with regard to its functions in general (55). New data gathered from meta-analysis suggest integration of three domains in the cingulate cortex: negative affect, pain, and cognitive control (56). This has shown that all three of these domains activate a common region within the aMCC. Since the aMCC might implement a domain-general process (56), we may hypothesise that self-regulation of the aMCC activity could be beneficial to any of those three domains. The feasibility of fMRI NFB in the aMCC was shown first by Weiskopf et al. (54) and later applied in the chronic pain domain (30).

\section{Application of Neurofeedback as a Supplementary Therapy Tool}

Neuroimaging already contributes to the treatment of mental disorders by providing information about the pathophysiological sources that may become the targets for physical (TMS, deep brain stimulation) or NFB intervention (57). NFB treatment can also be incorporated into a comprehensive biopsychosocial intervention package. However, we need to be aware that some neuropsychiatric diseases are heterogeneous; for example, depression may be brought about by focal lesions in the brain but can also occur as a result of a range of other mental disorders. Nevertheless, if a causal link is shown between mental illness and dysfunctional activity in specific areas or networks in the brain, the ability to self-regulate these areas may potentially have a favourable effect on a patient's mental health (57).

NFB using fMRI has recently been used to treat Parkinson's Disease (PD) (58). PD patients who underwent NFB training learned how to increase activity in the SMA using motor imagery and subsequently improved their motor speed of finger tapping, an overt movement. The transfer also generalised to their clinical ratings of motor symptoms, which improved after the NFB training. PD patients who did not receive feedback of their SMA activity did not gain control of SMA activation and consequently showed no motor improvement. This study has shown that selfregulation of motor circuits in PD patients through fMRI-NFB is achievable and may be clinically beneficial. In a proof-of-concept study of NFB in depression, depressed patients who underwent NFB using a positive emotion strategy not only learned to self-regulate emotion networks but also reduced their clinical symptoms (59). These studies are certainly promising, but randomised clinical trials would be needed to assess the clinical efficacy of NFB as supplementary therapy tool for these psychiatric diseases.

The use of NFB in clinical applications as a supplementary treatment is not limited to psychiatric diseases but could also be applied to lifestyle diseases such as obesity. The ability to intervene directly in the brain by voluntary regulation of eating-related regions could be used as a tool to increase the control of such brain regions and consequently influence eatingrelated behaviour. Exploratory work has been done recently where fMRI-NFB training of the anterior insula - a brain region involved in gustatory function - was investigated in lean and obese men (6o). The study found out that obese men were able to upregulate the anterior insula more significantly compared to lean men. This may suggest that obese men are more sensitive to gustatory learning; hence, future studies could be aimed at trying to downregulate activity in brain networks or areas involved in gustation and reward processing.

In the domain of chronic pain, an influential study has shown that healthy subjects were able to learn to control activation in the rostral ACC $(\mathrm{rACC})-\mathrm{a}$ region involved in pain perception and regulation (30). When subjects increased or decreased rACC activation, there was a significant change in the subjects' perception of pain caused by an externally applied noxious thermal stimulus. Moreover, pain perception was correlated with the level of rACC activation. This 
study has revealed that voluntary control over rACC activation mediated by fMRI-NFB leads to control over pain perception. The brain system that mediates pain perception is a highly relevant target for NFB training in a clinical setting. Chronic pain is an important clinical problem and is treated mainly with drugs which target neurons based on their selective expression of drug receptors. However, a drug may act on other receptors on other cells, which may bring about unwanted side effects. NFB using fMRI could be a promising supplementary, if not main, treatment, as it has not produced any detectable side effects, is less invasive, and most importantly, targets the "source" of a disease anatomically.

Despite numerous studies confirming the feasibility of fMRI-NFB and its clear advantages over EEG-NFB in many aspects such as higher spatial resolution, and better specificity, it is still rather mainly a research tool, while EEG still dominates in routine therapy due to its lower cost and higher availability. The lower temporal resolution of fMRI and the sluggish nature of the BOLD signal strongly reduce the contingency between the behavioural change (i.e. switching between strategies) and the feedback signal; therefore, NFB requires longer and probably more conscious training with fMRI than with EEG, which may also pose a limitation to the former in clinical applications. On the other hand, fMRI can be used to study brain areas hardly accessible via EEG (e.g. basal ganglia, hippocampus), and its indirect yet single measure (i.e. the BOLD signal) provides a lower degree of freedom in parameter selection (i.e. a more straightforward link to the brain functions) than the more direct but multiple measures (e.g. frequency bands, power, amplitude) of EEG. The issue of multiplicity can be well demonstrated by the abundance of EEG-NFB protocols determining which measure(s) should be trained and how $(61,62)$. Moreover, despite the longer history of EEG-NFB, the field has largely proceeded without validation until recently (63), methodologically satisfactory studies on its efficacy were lacking (64), and treatment effect is sometimes hard to distinguish from placebo effect (65). On the other hand, fMRI-NFB emerged from methodological research, and studies have usually been performed with careful controls as demonstrated in deCharms'study (30). We believe that these two techniques are complementary to each other, and while EEG-NFB may benefit from the more carefully controlled specificity of fMRI$\mathrm{NFB}$, the latter may benefit from the vast clinical experience of the former.

\section{Conclusion}

In this review, we have outlined the implementation of fMRI-NFB and highlighted that NFB is a promising tool that has both scientific and clinical applications. The feasibility of NFB training has been demonstrated in several domains of the brain, namely somatomotion, emotion and cognitive control. Studies of NFB on patient populations are starting to gain momentum. Nevertheless, there are still many open questions in various aspects of NFB and it would certainly be interesting to follow closely the developments of the field.

\section{Acknowledgement}

We would like to thank Biomedizinische NMR Forschungs GmbH (Prof Dr Jens Frahm) for providing the financial and administrative support for this project. Additionally, thanks to our colleague, Mr Blake Riley for his assistance with editing the manuscript.

\section{Conflict of Interest}

None.

Funds

German Federal Ministry for Education and Research (BMBF) via the Bernstein Focus Neurotechnologie (BFNT) Göttingen (Grant No. 01GQ0812).

\section{Authors' Contributions}

Conception and design, analysis and interpretation of the data, drafting of the article, critical revision of the article for the important intellectual content, final approval of the article, provision of study materials or patient, statistical expertise, obtaining of funding, administrative, technical or logistic support and collection and assembly of data: WID, TA

\section{Correspondence}

Ms Wan Ilma Dewiputri

BSc Biomedical Hons (Adelaide)

Biomedizinische NMR Forschungs GmbH

Max Planck Institute for Biophysical Chemistry

Am Fassberg 11

37077 Goettingen, Germany

Tel: +49-551 2011725

Fax: +49-551 2011307

Email: wan-ilma.dewiputri@mpibpc.mpg.de 


\section{General introduction}

Review Article | fMRI neurofeedback implementations and applications

\section{References}

1. Lubar JF, Shouse MN. Eeg and Behavioral-Changes in a Hyperkinetic Child Concurrent with Training of Sensorimotor Rhythm (SMR) - Preliminary-Report. Biofeedback Self-Reg. 1976;1(3):293-306.

2. Arns M, de Ridder S, Strehl U, Breteler M, Coenen A. Efficacy of Neurofeedback Treatment in ADHD: the Effects on Inattention, Impulsivity and Hyperactivity: a Meta-Analysis. Clin Eeg Neurosci. 2009;40(3):180-189.

3. Sterman MB, Egner T. Foundation and practice of neurofeedback for the treatment of epilepsy. App Psychophysiol Biofeedback. 2006;31(1):21-35.

4. Tan G, Thornby J, Hammond DC, Strehl U, Canady B, Arnemann K, et al. Meta-Analysis of EEG Biofeedback in Treating Epilepsy. Clin Eeg Neurosci. 2009;40(3):173-179.

5. Cox RW, Jesmanowicz A, Hyde JS. Real-time functional magnetic resonance imaging. Magnetic Reson Med. 1995;33(2):230-236.

6. Logothetis NK. What we can do and what we cannot do with fMRI. Nature. 2008;453(7197):869-878.

7. Logothetis NK, Pfeuffer J. On the nature of the BOLD fMRI contrast mechanism. Magn Reson Imaging. 2004;22(10): 1517-1531.

8. Logothetis NK, Pauls J, Augath M, Trinath T, Oeltermann A. Neurophysiological investigation of the basis of the fMRI signal. Nature. 2001;412(6843): $150-157$.

9. DeCharms RC. Applications of real-time fMRI. Nat Rev Neurosci. 2008;9(9):720-729.

10. Sitaram R, Weiskopf N, Caria A, Veit R, Erb M, Birbaumer N. fMRI Brain-Computer Interfaces: A tutorial on methods and applications. IEEE Signal Processing Magazine. 2008;25:95-106.

11. Weiskopf N. Real-time fMRI and its application to neurofeedback. Neuroimage. 2012;62(2):682-692.

12. Sulzer J, Haller S, Scharnowski F, Weiskopf $\mathrm{N}$, Birbaumer N, Blefari ML, et al. Real-time fMRI neurofeedback: Progress and challenges. Neuroimage. 2013;76:386-399.

13. Linden W, Moseley JV. The efficacy of behavioral treatments for hypertension. Appl Psychophysiol Biofeedback. 2006;31(1):51-63.

14. Maguire EA, Gadian DG, Johnsrude IS, Good CD, Ashburner J, Frackowiak RS, et al. Navigation-related structural change in the hippocampi of taxi drivers. Proc Natl Acad Sci U S A. 2000;97(8):4398-4403.

15. Maguire EA, Spiers HJ, Good CD, Hartley T, Frackowiak RS, Burgess N. Navigation expertise and the human hippocampus: a structural brain imaging analysis. Hippocampus. 2003;13(2):250-259.

16. Scholz J, Klein MC, Behrens TE, Johansen-Berg $\mathrm{H}$. Training induces changes in white-matter architecture. Nat Neurosci. 2009;12(11):1370-1371.
17. Ruiz S, Lee S, Soekadar SR, Caria A, Veit R, Kircher $\mathrm{T}$, et al. Acquired self-control of insula cortex modulates emotion recognition and brain network modulates emotion recognition and brain network
connectivity in schizophrenia. Hum Brain Mapp. 2011;34(1):200-212.

18. Lee S, Ruiz S, Caria A, Veit R, Birbaumer N, Sitaram R. Detection of Cerebral Reorganization Induced by Real-time fMRI Neurofeedback Training of Insula Activation: A Multivariate Investigation. Neurorehabil Neural Repair. 2011;25(3):259-267.

19. Zotev V, Krueger F, Phillips R, Alvarez RP, Simmons WK, Bellgowan P, et al. Self-regulation of amygdala activation using real-time fMRI neurofeedback. PLoS One. 2011;6(9):e24522.

20. Lee JH, Kim J, Yoo SS. Real-time fMRI-based neurofeedback reinforces causality of attention networks. Neurosci Res. 2012;72(4):347-354.

21. DeCharms RC, Christoff K, Glover GH, Pauly JM, Whitfield S, Gabrieli JDE. Learned regulation of spatially localized brain activation using real-time fMRI. Neuroimage. 2004;21(1):436-443.

22. Yoo SS, Lee JH, O'Leary $\mathrm{H}$, Panych LP, Jolesz FA. Neurofeedback fMRI-mediated learning and consolidation of regional brain activation during consolidation of regional brain activation during motor imagery. Int
2008;18(1):69-78.

23. Auer $\mathrm{T}$, Schweizer $\mathrm{R}$, Frahm J. The role of the anterior midcingulate cortex in neurofeedback training. Seattle (USA): 19th Annual Meeting of the Organization for Human Brain Mapping; 2013.

24. Schweizer R, Auer T, Frahm J. White matter changes associated with $f M R I$-based neurofeedback training. Seattle (USA): 19th Annual Meeting of the Organization for Human Brain Mapping; 2013.

25. Weiskopf N, Klose U, Birbaumer N, Mathiak K. Single-shot compensation of image distortions and BOLD contrast optimization using multi-echo EPI for real-time fMRI. Neuroimage. 2005;24(4):10681079.

26. Olsson CJ, Jonsson B, Nyberg L. Learning by doing and learning by thinking: an fMRI study of combining motor and mental training. Front Hum Neurosci. 2008;2:5.

27. Weiskopf N, Scharnowski F, Veit R, Goebel R, Birbaumer N, Mathiak K. Self-regulation of local Birbaumer N, Mathiak K. Self-regulation of local
brain activity using real-time functional magnetic resonance imaging (fMRI). J Physiol Paris. 2004;98(4):357-373.

28. Mathiak KA, Koush Y, Dyck M, Gaber TJ, Alawi E Zepf FD, et al. Social reinforcement can regulate localized brain activity. Eur Arch Psychiatry Clin Neurosci. 2010;260(2 Suppl):S132-S136.

29. Caria A, Sitaram R, Veit R, Begliomini C, Birbaumer N. Volitional Control of Anterior Insula Activity Modulates the Response to Aversive Stimuli. A RealTime Functional Magnetic Resonance Imaging Study. Biol Psychiatry. 2010;68(5):425-432. 
30. DeCharms RC, Maeda F, Glover GH, Ludlow D, Pauly JM, Soneji D, et al. Control over brain activation and pain learned by using real-time functional MRI. Proc Natl Acad Sci USA. 2005;102(51):1862618631.

31. Mathiak K, Posse S. Evaluation of motion and realignment for functional magnetic resonance imaging in real time. Magn Reson Med. 2001;45(1):167-171.

32. Cox RW, Jesmanowicz A. Real-time 3D image registration for functional MRI. Magn Reson Med. 1999;42(6):1014-1018.

33. Weiskopf N, Klose U, Birbaumer N, Mathiak K. Single-shot compensation of image distortions and BOLD contrast optimization using multi-echo EPI for real-time fMRI. Neuroimage. 2005;24(4):10681079 .

34. Gao K, Posse S. TurboFIRE: Real-time fMRI with automated spatial normalization and Talairach Daemon database. Seattle (USA): 19th Annual Daemon database. Seattle (USA): 19th Annual
Meeting of the Organization for Human Brain Mapping; 2013.

35. Hinds O, Ghosh S, Thompson TW, Yoo JJ, WhitfieldGabrieli S, Triantafyllou C, et al. Computing moment-to-moment BOLD activation for real-time neurofeedback. Neuroimage. 2011;54(1):361-368.

36. Nakai T, Bagarinao E, Matsuo K, Ohgami Y, Kato C. Dynamic monitoring of brain activation under visual stimulation using fMRI--the advantage of real-time fMRI with sliding window GLM analysis. J Neurosci Methods. 2006;157(1):158-167.

37. Voyvodic JT. Real-time fMRI paradigm control, physiology, and behavior combined with near realtime statistical analysis. Neuroimage. 1999;10(2):91106.

38. Posse S, Binkofski F, Schneider F, Gembris D, Frings W, Habel U, et al. A new approach to measure singleevent related brain activity using real-time fMRI: feasibility of sensory, motor, and higher cognitive tasks. Hum Brain Mapp. 2001;12(1):25-41.

39. Goebel R. Cortex-based real-time fMRI. Neuroimage. 2001;13(6):S129.

40. Esposito F, Seifritz E, Formisano E, Morrone R, Scarabino T, Tedeschi G, et al. Real-time independent component analysis of fMRI time-series. Neuroimage. 2003;20(4):2209-2224.

41. Haynes JD, Rees G. Decoding mental states from brain activity in humans. Nat Rev Neurosci. 2006;7(7):523-534.

42. LaConte SM, Peltier SJ, Hu XP. Real-time fMRI using brain-state classification. Hum Brain Mapp. 2007;28(10):1033-1044.

43. Poldrack RA. Region of interest analysis for fMRI. Soc Cogn Affect Neur. 2007;2(1):67-70.

44. Johnston SJ, Boehm SG, Healy D, Goebel R, Linden DEJ. Neurofeedback: A promising tool for the selfregulation of emotion networks. Neuroimage. 2010;49(1):1066-1072.
45. Caria A, Veit R, Sitaram R, Lotze M, Weiskopf $\mathrm{N}$, Grodd W, et al. Regulation of anterior insular cortex activity using real-time fMRI. Neuroimage. 2007;35(3):1238-1246.

46. Yoo S-S, Jolesz FA. Functional MRI for neurofeedback: feasibility study on a hand motor task. Neuroreport. 2002;13(11):1377-1381.

47. Berman BD, Horovitz SG, Venkataraman G, Hallett M. Self-modulation of primary motor cortex activity with motor and motor imagery tasks using realtime fMRI-based neurofeedback. Neuroimage. 2012;59(2): $917-925$.

48. Walsh M, Anderson JR. Modulation of the feedbackrelated negativity by instruction and experience. Proc Natl acad Sci U S A. 2011;108(47):1904819057.

49. Auer T, Frahm J. Confounding factors in neurofeedback training based on fMRI of moto imagery. Neurosci Lett. 2011;500(Supp):e32. doi: 10.1016/j.neulet.2011.05.160.

50. AuerT, Schweizer R, Frahm J. Predicting the success of neurofeedback training based on fMRI of imaginary movement. Beijing $(\mathrm{CN})$ : 18th Annual Meeting of the Organization for Human Brain Mapping; 2012.

51. Posse S, Fitzgerald D, Gao K, Habel U, Rosenberg D, Moore GJ, et al. Real-time fMRI of temporolimbic regions detects amygdala activation during singletrial self-induced sadness. Neuroimage. 2003;18(3): $760-768$.

52. Hamilton JP, Glover GH, Gotlib IH. Healthy individuals can use real-time fMRI neurofeedback to individuals can use real-time fMRI neurofeedback to
modulate activity in the subgenual anterior cingulate cortex. Biol Psychiatry. 2007;61(8):30s.

53. Hamilton JP, Glover GH, Hsu J-J, Johnson RF, Gotlib IH. Modulation of subgenual anterior cingulate cortex activity with real-time neurofeedback. Hum Brain Mapp. 2011;32(1):22-31.

54. Weiskopf N, Veit R, Erb M, Mathiak K, Grodd W, Goebel R, et al. Physiological self-regulation of regional brain activity using real-time functional magnetic resonance imaging (fMRI): methodology and exemplary data. Neuroimage. 2003;19(3):577586.

55. Vogt BA. Cingulate Neurobiology and Disease. New York (US): Oxford University Press; 2009.

56. Shackman AJ, Salomons TV, Slagter HA, Fox AS, Winter JJ, Davidson RJ. The integration of negative affect, pain and cognitive control in the cingulate cortex. Nat Rev Neurosci. 2011;12(3):154-167.

57. Linden DEJ, Fallgatter AJ. Neuroimaging in psychiatry: from bench to bedside. Front Hum Neurosci. 2009;3:49.

58. Subramanian L, Hindle JV, Johnston S, Roberts MV, Husain M, Goebel R, et al. Real-Time Functional Magnetic Resonance Imaging Neurofeedback for Treatment of Parkinson's Disease. J Neurosci. 2011;31(45):16309-16317. 


\section{General introduction}

Review Article | fMRI neurofeedback implementations and applications

59. Linden DEJ, Habes I, Johnston SJ, Linden S, Tatineni R, Subramanian L, et al. Real-Time Self-Regulation of Emotion Networks in Patients with Depression. PLoS One. 2012;7(6): 38115

6o. Frank S, Lee S, Preissl H, Schultes B, Birbaumer N, Veit R. The Obese Brain Athlete: Self-Regulation of the Anterior Insula in Adiposity. PLoS One. 2012;7(8):e42570.

61. Hammer BU, Colbert AP, Brown KA, Ilioi EC. Neurofeedback for insomnia: a pilot study of Z-score SMR and individualized protocols. Appl Psychophysiol Biofeedback. 2011;36(4):251-264.

62. Micoulaud-Franchi JA, Bat-Pitault F, Cermolacce M, Vion-Dury J. Neurofeedback for attentiondeficit/hyperactivity disorder: From efficacy to neurophysiology specificity effect. Ann Med-Psychol. 2011;169(3):200-208.
63. Gruzelier J, Egner T, Vernon D. Validating the efficacy of neurofeedback for optimising performance. Prog Brain Res. 2006;159:421-431.

64. Drechsler R. Is Neurofeedback Training an Efficaciou Treatment for ADHD? A Review of Recent Findings. $Z$ Neuropsychol. 2011;22(2):131-146.

65. Becerra J, Fernandez T, Roca-Stappung M, DiazComas L, Galan L, Bosch J, et al. Neurofeedback in healthy elderly human subjects with electroencephalographic risk for cognitive disorder. $J$ Alzheimer's Dis. 2012;28(2):357-367. 


\section{Functional localization of the anterior mid-cingulate cortex for real-time fMRI neurofeedback}

\subsection{Introduction}

Neurofeedback (NF) is a method to achieve self-regulation of brain states via information about own ongoing brain activity which can be provided by means of rtfMRI (Weiskopf, 2012). Rt-fMRI NF enables subjects to gain control of their own brain state when provided with real-time feedback from a target brain region or a network (Ruiz et al., 2014). The first step in a rt-fMRI NF experimental framework is to define a target region-of-interest (ROI). A target ROI can be defined from its anatomy if the brain region's anatomical landmarks are well-defined for example for the sensorimotor areas; or functionally, on the basis of neural mechanism underlying the desired behavioral change if the region has no well-defined anatomical landmarks, for example higher cognitive areas (Sulzer et al., 2013). The aMCC is intended to be used as the target ROI. NF studies of the anterior cingulate cortex (ACC) have mostly employed a functional localizer task in the domain that is to be probed in the main NF experiment, e.g. pain localizer task to investigate NF of pain regulation in rostral ACC (deCharms et al., 2005), or an emotional localizer task to study NF of emotion regulation in the subgenual ACC (Hamilton et al., 2011; Linden et al., 2012).

\subsubsection{The many facets of the anterior mid-cingulate cortex}

The aMCC is a region of the cingulate cortex where negative emotion, pain, cognitive control, and intentional motor control are functionally and anatomically integrated (Shackman et al., 2011; Hoffstaedter et al., 2013) (Figure 1). On the network level, the

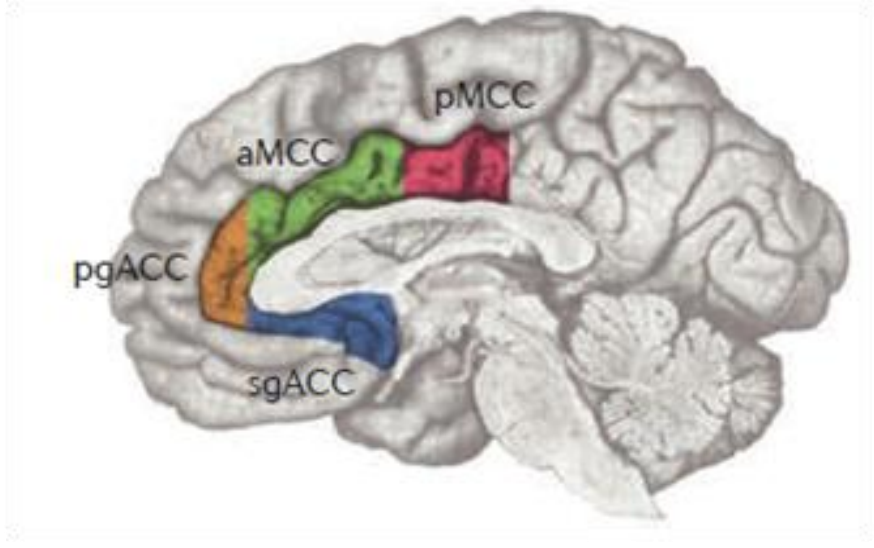

and triggers cognitive control signal (Menon and Uddin, 2010). The cognitive control
Figure 1: The major subdividions of the cingulate cortex.

Reprinted by permission from Macmillan Publishers Ltd: Nature Reviews Neuroscience (Shackman et al., 2011) copyright (2011).

aMCC is part of the salience network, which monitors salient internal and external events (Seeley et al., 2007) 
domain of the aMCC includes neural processes such as sustained attention, response inhibition and conflict resolution. One behavioral test most often used in the domain of cognitive control is the Continuous Performance Task (CPT). The CPT is designed to investigate sustained attention, response inhibition, and anticipation, and is generally characterized by successive stream of rapid stimulus presentation with a designated "target" stimulus or "target" pattern (Riccio et al., 2002) to which the subject has to react with a motor response (e.g. button press).

\subsubsection{Analyzing the aMCC}

For the subsequent main NF study, the aMCC is to be targeted based on its function in the domain of cognitive control, and the CPT, (through examining its anticipation phase) have been chosen as a functional localizer task for the aMCC. To analyze the CPT fMRI data, this study used the general linear model (GLM), a hypothesis-driven analysis method; and independent components analysis (ICA), a data-driven method. The GLM is an established method, widely used in analyzing fMRI data and requires a model about the data. On the other hand, the essence of ICA is its ability to separate sources of neural signal, structured noise, and random noise into separate spatiotemporal independent components without requiring a priori assumptions (Beckmann and Smith, 2013). Therefore, the model-free ICA can be used as a data-denoising step.

\subsubsection{Aims}

The aim of this study is to establish functional localization methods for the region of cognitive control of the aMCC in individuals, with the further goal to use it in rt-fMRI NF studies. Specifically in the current study, CPT with rt-fMRI was used, and two fMRI analyses: the standard GLM, and ICA incorporating GLM, were compared. 


\subsection{Materials and methods}

\subsubsection{Participants}

Eleven healthy right-handed subjects (5 females) of average age $25.3 \pm 2.3$ years old participated in the study. Subjects gave their written informed consent before the study and received EUR 10 per hour for their participation. The study was approved by the local ethics committee of Georg-Elias-Müller-Institute for Psychology, at the University of Göttingen. Subjects underwent two MRI sessions on different days: a pre-localizer session consisting of a high-resolution anatomical MRI scan, and the main session consisting of the functional localizer task. Data of one subject (male, 25) was not used in the subsequent offline fMRI analysis due to inconsistent task performance during data acquisition.

\subsubsection{MRI parameters}

All MR images were acquired on a 3T Tim Trio MRI scanner (Siemens Healthcare, Erlangen, Germany) using a 32-channel head coil for signal reception. Structural wholebrain T1-weighted MRI was obtained by an inversion-recovery 3D FLASH sequence (TR $=2530 \mathrm{~ms}, \mathrm{TE}=3.26 \mathrm{~ms}$, flip angle $=7^{\circ}, \mathrm{TI}=1100 \mathrm{~ms}$ ) at $1.0 \times 1.0 \times 1.0 \mathrm{~mm}^{3}$ isotropic resolution. All BOLD fMRI measurements were obtained by a gradient-echo EPI sequence $\left(\mathrm{TR}=2 \mathrm{~s}, \mathrm{TE}=36 \mathrm{~ms}\right.$, flip angle $=70^{\circ}$, acquisition matrix $\left.=96 \times 96\right)$ at $2.0 \times 2.0$ $\times 4.0 \mathrm{~mm}^{3}$ resolution with 22 axial slices oriented along the AC-PC line, and encompassing the cerebrum until the midbrain level. To reduce geometric distortions of EPI scans, a field map scan was acquired (TR $=468 \mathrm{~ms}$, TE $1=4.92 \mathrm{~ms}$, TE $2=7.38 \mathrm{~ms}$ ). A single whole-brain EPI measurement with the same orientation and resolution as the fMRI measurements was obtained ( $\mathrm{TR}=3250 \mathrm{~ms}, \mathrm{TE}=36 \mathrm{~ms}$, flip angle $=70^{\circ}, 36$ slices $)$ to optimize registration of the partial-brain fMRI measurements to the structural whole brain scan. Motion correction on fMRI data was performed in k-space (online software of the manufacturer).

\subsection{3 rt-fMRI}

Online rt-fMRI monitoring was implemented via real-time data export from the MR scanner computer to the rt-fMRI analysis computer (Dewiputri and Auer, 2013). Online rt-fMRI analysis was performed in Turbo Brain Voyager (TBV) software (Brain Innovation, Maastricht, Netherlands) which included data pre-processing (motion correction, spatial smoothing using a Gaussian kernel of $8.0 \mathrm{~mm}$ FWHM) and computation of statistical maps from GLM contrasts: cue > distractor. Head motion and 
inconsistent task performance were closely monitored during the measurement. If such behavior was detected, the subject was asked to repeat the fMRI run after re-advising them about head motion and the task.

\subsubsection{Paradigm and task}

The CPT O-X-H paradigm was implemented as the functional localizer task. Visual stimuli were projected onto a screen inside the MRI scanner, and viewed by the subjects through a mirror mounted on top of the head-coil. The visual stimuli were presented using Presentation software (NeuroBehavioral Systems Inc, Berkeley, USA) and consisted of the white letter $\mathrm{O}, \mathrm{X}$, or $\mathrm{H}$ shown on a black background (Figure 2). Each letter was presented for $250 \mathrm{~ms}$ and the interstimulus interval was $5750 \mathrm{~ms}$. There were 80 stimuli in one run. Prior to the onset of the first stimuli, a baseline of 8 seconds was included. One run of the CPT paradigm lasted 8.13 minutes (244 fMRI volumes). Throughout the whole run, occurance of a cue (0) has a 40\% probability; while occurance of the target $(\mathrm{X})$ and the distractor $(\mathrm{H})$ have a probability of $30 \%$ each. From this $30 \%$, two-thirds of the occurrence was either a cue-target $(\mathrm{O}-\mathrm{X})$ or a cue-distractor (O-H) pair while one-third of the occurrence was an uncued X or H. Subjects were instructed to press the response button with their right thumb only for a cued target (an $\mathrm{X}$ that is preceded by an 0 ), and to refrain from pressing the button when a cued distractor (an $\mathrm{H}$ preceded by an 0 ) was presented.

Subjects were instructed to be as fast and as accurate as possible. Only correct responses to targets which occured within $1000 \mathrm{~ms}$ of stimulus presentation were counted. Feedback on the results was shown on the screen at the end of the run. Before performing the task, a simulation of the CPT was shown to the subjects outside the scanner to help them understand the task.

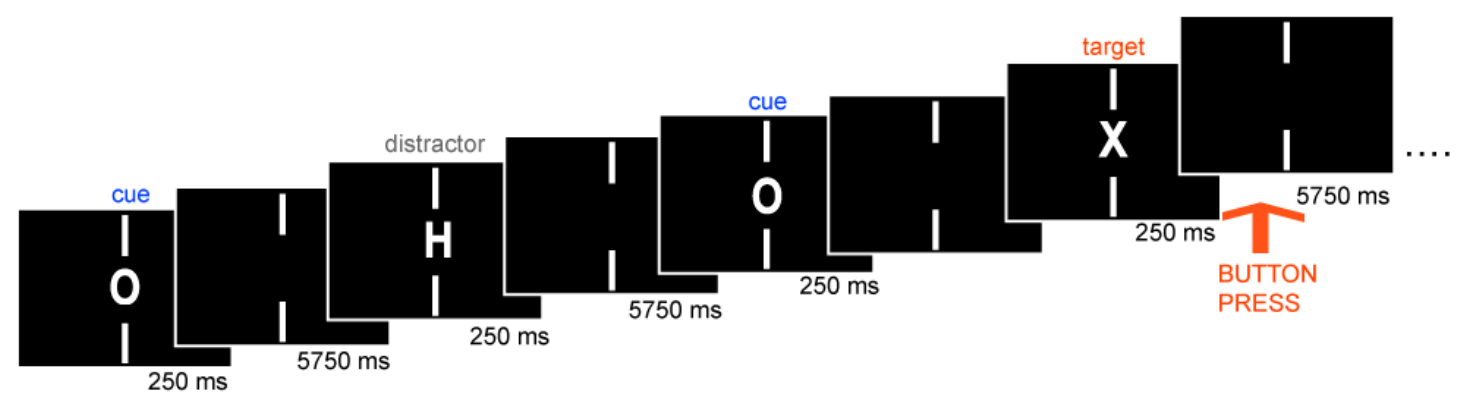

Figure 2: CPT paradigm. Subjects were instructed to only press the button if the target $(\mathrm{X})$ is preceded by the cue (O); i.e. cued target, but to refrain from it otherwise. 


\subsubsection{Offline data analysis}

\subsubsection{Pre-processing}

fMRI data processing was carried out within FEAT (FMRI Expert Analysis Tool) Version 5.98, part of FSL (FMRIB's Software Library, www.fmrib.ox.ac.uk/fsl). Pre-statistics processing included motion correction using MCFLIRT (Jenkinson et al., 2002); fieldmap-based EPI unwarping using PRELUDE+FUGUE (Jenkinson, 2003); non-brain removal using BET (Smith, 2002); spatial smoothing using a Gaussian kernel of FWHM 5 $\mathrm{mm}$; grand-mean intensity normalization of the entire 4D dataset by a single multiplicative factor; high-pass temporal filtering (Gaussian-weighted least-squares straight line fitting, with sigma $=15 \mathrm{~s}$ ).

\subsubsection{GLM analysis}

Model-based fMRI analysis was carried out using FEAT (FMRI Expert Analysis Tool) Version 5.98, part of FSL (FMRIB's Software Library, www.fmrib.ox.ac.uk/fsl). Timeseries statistical analysis was carried out using FILM with local autocorrelation correction (Woolrich et al., 2001). The data was modeled in the GLM by two conditions: 4-6 seconds after cue (letter 0) and 4-6 seconds after all distractors (letter $\mathrm{H}$ ). The contrast used to define aMCC in this analysis was cue > distractor. $Z$ (Gaussianised T/F) statistic images were thresholded using clusters determined by $z>3.1$ and a corrected cluster significance threshold of $p=0.05$ (Worsley, 2001). FLIRT was used to register fMRI image to high resolution structural and/or standard space images (Jenkinson 2001, 2002 (Jenkinson and Smith, 2001; Jenkinson et al., 2002)). Registration from high resolution structural to standard space was then further refined using FNIRT nonlinear registration (Andersson et al., 2007). AMCC clusters were identified by visual inspection of the activation map of the cue $>$ distractor contrast.

\subsubsection{ROI identification by ICA-GLM analysis}

In addition to model-based GLM analysis, CPT fMRI data were analyzed with singlesession Probabilistic ICA (Beckmann and Smith, 2004) as implemented in MELODIC (Multivariate Exploratory Linear Decomposition into Independent Components) Version 3.12, part of FSL (FMRIB's Software Library, www.fmrib.ox.ac.uk/fsl). The following analysis steps were performed as described in the standard FSL ICA output (Beckmann and Smith, 2004): Pre-processing consisted of masking of non-brain voxels, voxel-wise de-meaning of the data, normalisation of the voxel-wise variance. Pre-processed data were whitened and projected into a $n$-dimensional subspace using probabilistic 
Principal Component Analysis where the $n$ (average: 46 components) was estimated using the Laplace approximation to the Bayesian evidence of the model order (Minka, 2000; Beckmann and Smith, 2004). The whitened observations were decomposed into sets of vectors which describe signal variation across the temporal domain (timecourses) and across the spatial domain (maps) by optimizing for non-Gaussian spatial source distributions using a fixed-point iteration technique (Hyvärinen, 1999). Estimated Component maps were divided by the standard deviation of the residual noise and thresholded by fitting a mixture model to the histogram of intensity values (Beckmann and Smith, 2004).

Additionally, as an option in MELODIC, the time course of each Independent Component (IC) was entered into a GLM for post-hoc analysis of the cue > distractor contrast. To help in the identification of a task-related component, IC maps whose pvalues are $<0.05$ for the cue $>$ distractor GLM contrast were classified as a potential task-related IC. Exclusion of noise-related IC maps was performed by visual inspection of the potential task-related IC, based on available guidelines (Kelly Jr et al., 2010). In each subject the aMCC was identified individually from one IC map. If the aMCC was present in more than one task-related IC maps, the IC map which showed activation of aMCC as part of the salience network was chosen.

\subsubsection{Individual definition of the aMCC}

The localization of the aMCC at the individual level was done on 34 additional subjects recruited for the main rt-fMRI neurofeedback study targeting the aMCC. Please see Section 3.2.1 for more details about the subjects.

The IC map containing the aMCC cluster was individually thresholded at the highest threshold at which cluster of contiguous voxels within the later refined aMCC area can be clearly defined ( $\mathrm{p}=0.05-0.30)$. Thresholded IC maps in the native space were registered to the standard space using FLIRT (Jenkinson and Smith, 2001; Jenkinson et al., 2002), to enable easier delineation of aMCC based on the standard space atlas and to define the shape and extent of the aMCC clusters. The ROI was drawn manually on the thresholded IC maps in the native space on axial slices, while taking the standard space IC maps as guidance. The ROI was drawn within anatomical limits as defined by Vogt and Shackman (Vogt, 2009; Shackman et al., 2011) who describe the aMCC as the portion of the cingulate gyrus and the paracingulate gyrus superior to the anterior mid-body of the corpus callosum. 


\subsection{Results}

\subsubsection{Behavioral data}

The reaction time for the CPT was $434.2 \mathrm{~ms} \pm 14.7 \mathrm{~ms}$ (mean \pm SEM) and the accuracy was $99 \% \pm 0.25 \%$ (mean \pm SEM). This showed that subjects had an adequately high behavioral performance in the CPT.

\subsubsection{GLM fMRI analysis}

The model-based GLM-analysis with FEAT detected a cluster aMCC activation of reasonable size in both hemispheres in seven out of ten subjects after performing one run of CPT. Optimal aMCC detection in CPT is shown in Figure 3A. Apart from the aMCC cluster the following areas were also activated in the cue > distracter contrast: bilateral insula, basal ganglia, SMA, thalamus, left motor cortex (Figure 5). Figure 3B-D shows data from the three subjects in which the detection of aMCC was suboptimal. Lowering the threshold to $z>2.0$ enabled the detection of only minor aMCC activation cluster as in Figure 3C; but not for the data in Figure 3D.

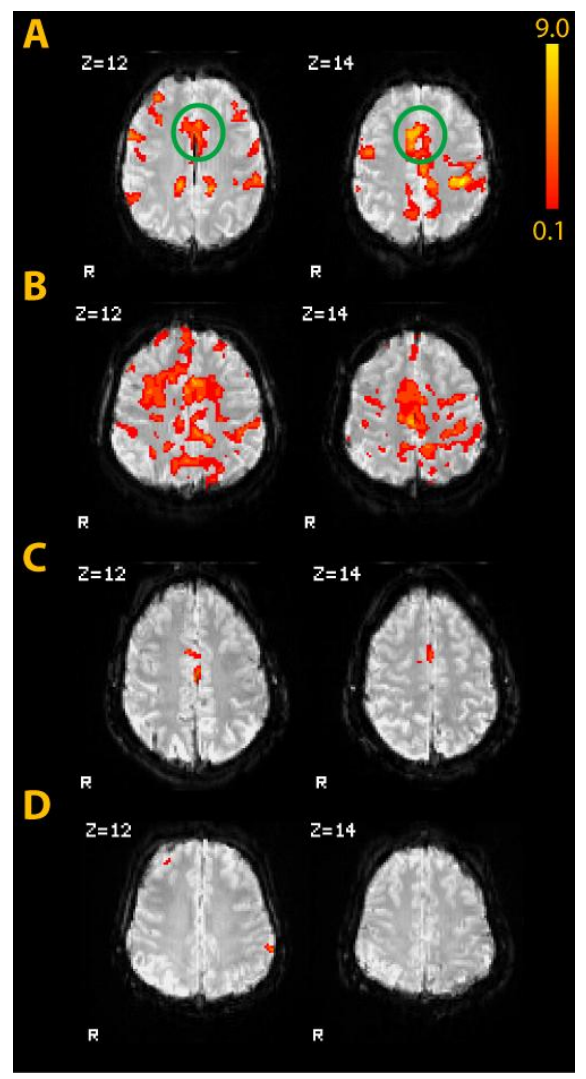

Figure 3: Single subject example results in GLM analysis of CPT

A. aMCC (green circle) of reasonable cluster size

B. Results contaminated by noise

C. aMCC cluster size too small

D. No aMCC detected

$\mathrm{z}=$ axial slice

\subsubsection{ICA-GLM fMRI analysis}

The GLM-based analysis of the fMRI data was not able to detect aMCC in all 10 subjects. Therefore, ICA-GLM was introduced as a denoising procedure. The analysis with ICA 
generated on average 46 IC maps (range: 36-63) in individuals. By performing a GLM on the time courses of the ICs, one IC map showing an aMCC activation cluster spanning both hemispheres was able to be detected in each of the 10 subjects (Figure 4). To allow the comparison of the GLM and ICA-GLM results (which uses different thresholding method), activation maps in Figure 2 and 3 are color-coded with a fixed range between $z$ $=0.1-9.0$.

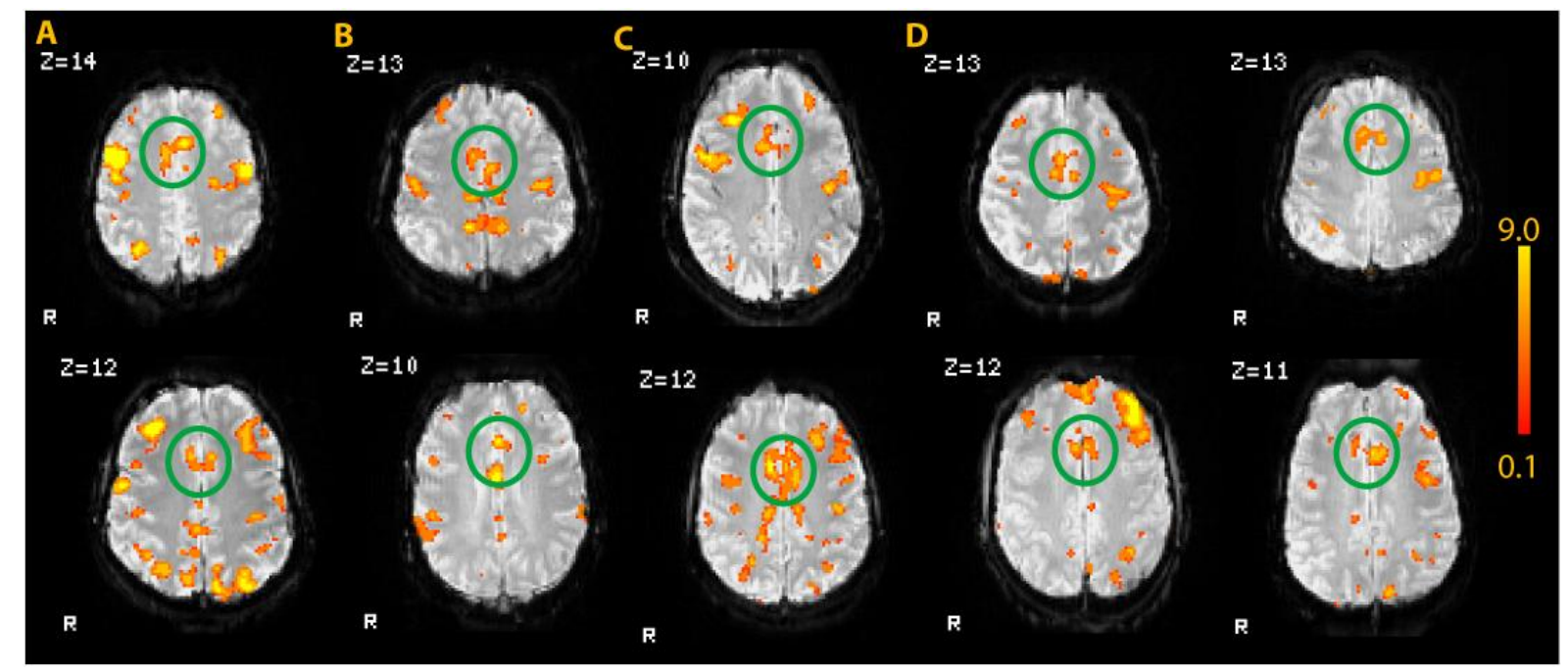

Figure 4: Detection of aMCC (green circle) from IC maps in native low-resolution fMRI space. The labels A-D correspond to the subjects labeled in Figure 2. $z$ = axial slice

\subsection{4 aMCC ROI definition}

The CPT paradigm and ICA-GLM method were then applied to the subjects of the main NF experiment (Section 3). The aMCC was able to be detected and defined in 30 subjects. However, aMCC was not optimally detected in four subjects who were subsequently excluded from the NF study due to the following reasons: in one subject, it was the combination of having a small cluster size and a relatively large interhemispheric fissure dividing the aMCC, which could impede BOLD signal extraction for the NF study; and in the other three, spurious BOLD activations in the CPT that was first detected by the rt-fMRI online monitoring and later confirmed in the offline analyses. The defined ROI in all individuals are shown in Figure 5. The coordinates of the mean peak voxel and center of gravity of the aMCC cluster in MNI space $(\mathrm{mm})$ are $\mathrm{x}=$ $8, y=26, z=30$ and $x=1 y=23 z=33$ respectively. The size of the ROI defined according to this method was $233 \pm 54$ voxels (mean \pm SD). Similar to the GLM analysis, aMCC activation was often found in task-related IC maps together with various degrees of activations of bilateral insula, SMA, and basal ganglia. 


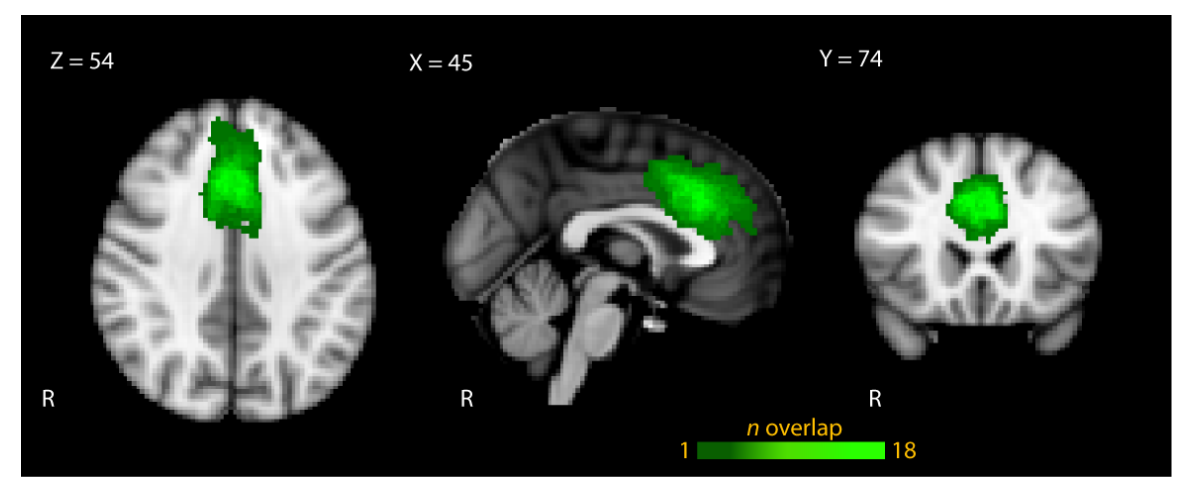

Figure 5: Overlap of the aMCC ROI of 30 subjects of the neurofeedback study in standard MNI space. Color bar indicates number of subjects. $z=$ axial slice, $x=$ sagittal, $y=$ coronal slice.

\subsection{5 rt-fMRI monitoring}

Besides the offline analysis of the fMRI data, rt-fMRI also allows to monitor the CPT run online. An example of the online rt-fMRI monitoring of two CPT runs with rt-fMRI from the same subject is shown in Figure 6. Some head motion is evident (Figure 6A) from the erratic motion correction plot, and represented in the statistical map by positive activations in the ventricles. After the subject was advised to keep still when repeating the task, activations in the ventricles were no longer seen (Figure 6B). Figure 6B also shows the expected brain activations for the cue > distractor contrast in the following brain areas: aMCC, bilateral insula, supplementary motor area (SMA) and left motor cortex. In the present study, this occurred in one subject whose dataset was excluded from offline analysis. In the main NF study, three subjects were excluded due to spurious BOLD activations in the CPT, which was first detected by the rt-fMRI online monitoring and later confirmed by offline analysis. 


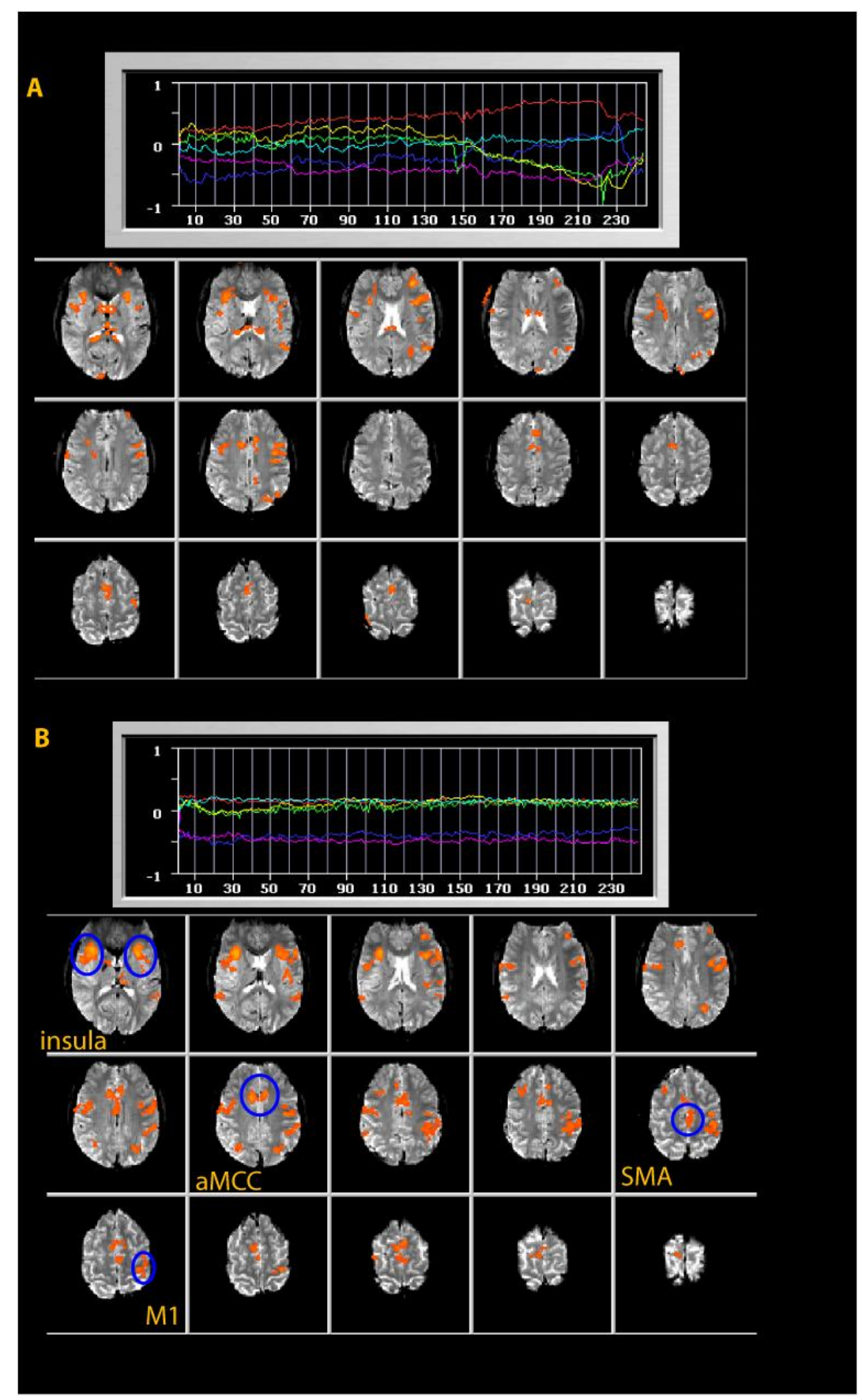

Figure 6: Monitoring the functional localizer scan by rt-fMRI in one subject. In this example, TBV interface is used to monitor spurious activations, artifacts, and head motion. The fMRI run in $A$. has considerable head motion compared to $\mathbf{B}$. 


\subsection{Discussion}

The present work has established CPT as a suitable for functional localization of the aMCC in the domain of cognitive control, and ICA-GLM as a suitable analysis method for the same purpose. In addition, simultaneous monitoring of the functional localizer task with rt-fMRI is recommended.

\subsubsection{GLM and ICA-GLM analysis of the functional localizer task}

The criteria of a suitable functional localizer task to detect aMCC would be that the task should be able to activate the aMCC reliably in every individual. A suitable analysis method for the functional localizer task should be one that is sensitive to detect aMCC as a cluster of reasonable size in each individual with the least amount of noise. The current work in this chapter presents the CPT as a functional localizer task, in conjunction with single-subject ICA-GLM analysis, as a suitable method to localize aMCC.

Measuring neuronal activity in fMRI is challenging as the BOLD signal has contributions from many sources including breathing, head motion and scanner artifacts. Pre-processing the fMRI data helps eliminate such effects, however, residual noise that has both spatial and temporal structures can still remain and can negatively affect the signal-to-noise ratio, sensitivity and specificity of results obtained by BOLD fMRI. This is where ICA functions a useful tool to denoise fMRI data (Beckmann, 2012). The data of the current study shows that ICA increased the sensitivity to detect aMCC in all ten subjects, compared to only seven in the model-based GLM analysis.

The increased sensitivity to detect brain activations comes at a cost of time. One needs to do visual inspection of the IC maps to distinguish signal from noise. This could mean that an inexperienced researcher would first need to learn how to do so but fortunately there are published guidelines available (Kelly Jr et al., 2010). Automated methods of classifying ICs have been developed, but from my later experience using automated methods, I find that one still needs to visually inspect the results of those automated classification.

The classic fMRI analysis using GLM is still a reliable method. If a suitable model is specified, analyzing fMRI data with a GLM is usually able to produce statistical maps that signify where the brain has activated in response to the stimulus. However, in cases where the GLM does not work as expected, trying the ICA is recommended. For example, when expected results are still unobtainable even after lowering the threshold of the 
statistical map, when noise still persist in the data after pre-processing, or when higherlevel GLM (which would boost sensitivity) is not performed for types of study where single-subject analysis is required.

The ICA-GLM approach harnesses the strength of signal source separation in ICA and the simplicity (and familiarity) of the GLM. Design matrix and contrasts normally used in a GLM analysis can be used post-hoc in ICA to determine potentially task-related IC maps. From my experience, specifying a model in post-hoc ICA helps in reducing the number of ICs that need to be visually inspected in order to select the aMCC from potentially task-related ICs. For the purpose of functional localization, one run of CPT analyzed with the ICA-GLM method was enough to identify the aMCC in each individual. The concept of combining hypothesis-driven and data-driven methods have also been used in localization of language regions in patients with tumors (Caulo et al., 2010).

\subsubsection{Activation of alMCC as part of a cognitive network}

In the GLM analysis, aMCC cluster was often seen together in the same activation map with SMA, bilateral insula, basal ganglia, and thalamus. One might argue about the specificity of the CPT as a functional localizer for aMCC, however the functional localizer task does need to isolate only the target region, it should just be sensitive enough to detect activity, which spans an area of reasonable shape and size in the target region to allow localization. Furthermore cognitive tasks rarely activate just a single region. In ICA-GLM analysis, aMCC cluster was seen in task-related IC maps of the salience network (aMCC, bilateral insula) and the somatomotor network (SMA, premotor, somatomotor cortex). The ROI is best defined from the network whose function one intends to probe in the main NF experiment.

\subsubsection{Functional localizer task guided by rt-fMRI}

Monitoring the functional localizer scan with rt-fMRI system confers many advantages. It acts as a quality control procedure to screen subjects for the main experiment, whereby subjects who show too much head movements, or spurious BOLD activations in the localizer task can be excluded at the functional localizer stage before entering the main experiment. The decision to exclude such subjects made during the functional localizer rt-fMRI run would save the experimenter's time whereby the experimenter can already decide to recruit new subjects rather than having to wait for the results of the offline analyses. Nevertheless, additionally, the whole GLM and ICA-GLM analyses were performed to confirm the decision on the excluded subjects. Therefore, based on my 
experience, running the functional localizer scan in conjunction with rt-fMRI is an essential step. Indeed, this step helped tremendously in selecting subjects who are less likely to be problematic for the main rt-fMRI neurofeedback study. The activation maps obtained from the more detailed offline fMRI analysis (Figure 3A) showed similar areas being activated as in the online rt-fMRI analysis (Figure 6B). This tells us that the results seen during the online rt-fMRI run (if all parameters are set correctly) of the functional localizer task can give an impression about the results that would be obtained in the offline analysis.

\subsection{Conclusions}

The present work in this chapter has shown that CPT is a suitable paradigm for functional localization of the aMCC. The ICA-GLM analysis method increased sensitivity to localize aMCC at the individual level. Rt-fMRI monitoring of the functional localizer scan is helpful for quality control. 
rt-fMRI NF of aMCC 


\section{Rt-fMRI cognitive neurofeedback training of the aMCC}

\subsection{Introduction}

\subsection{1 rt-fMRI neur ofeedback}

Neurofeedback (NF) is a variation of biofeedback that facilitates subjects to gain control of an aspect of their own brain activity. These aspects range from up-and-down regulation of a single brain area (Weiskopf et al., 2004; Caria et al., 2010), interhemispheric differences of two homologous area (Robineau et al., 2014), to connectivity between multiple brain regions (Koush et al., 2013). These aspects can be measured by various modalities such as frequencies and slow cortical potentials (SCP) in electroencephalography (EEG) (Niv, 2013); or BOLD activity in fMRI (Weiskopf, 2012; Sulzer et al., 2013), and more recently in fNIRS (Cutini and Brigadoi, 2014). In NF, the modality of the feedback is mostly visual and can be a "thermometer display", graph, or virtual reality avatar. The task for the subjects is to search for a mental strategy to achieve self-regulation of an aspect of their brain activity, usually mental imagery related to the function of the area or network being targeted. Subjects then receive concurrent feedback of their brain activity in near real-time. Through trial-and-error and operant conditioning during the NF training period, they learn to prune their strategy to reach the desired outcome.

$\mathrm{NF}$ is primarily conducted using EEG which has high temporal resolution but poor spatial resolution. The advent of real-time functional magnetic resonance imaging (rt-fMRI) (Cox, 1995), has encouraged growth in fMRI NF studies (Sulzer et al., 2013). This owes to the advantages of fMRI, having higher spatial resolution and allowing subcortical coverage, surpassing that of EEG. Methodological advances in fMRI have enabled instantaneous access to experimental results by analyzing data as soon as they are acquired - an essential requirement for rt-fMRI NF setup. Ever since then, various brain areas have been targeted in rt-fMRI NF, ranging from the motor (DeCharms et al., 2004; Yoo et al., 2008; Auer and Frahm, 2011; Berman et al., 2012; Johnson et al., 2012), emotion (Caria et al., 2007; Caria et al., 2010; Hamilton et al., 2011; Zotev et al., 2011; Veit et al., 2012; Ruiz et al., 2013), to cognitive brain areas (Mathiak et al., 2010). The majority rt-fMRI NF studies have been done at the level of a single region, and more recently at the level of networks (Koush et al., 2013). In addition, various populations have been used, ranging mostly from healthy normal subjects, to clinical populations (deCharms et al., 2005; Subramanian et al., 2011; Linden et al., 2012; Ruiz et al., 2013). 
These developments are already far-reaching, however there are still many open questions including those to address the fundamentals.

This study seeks to address open questions on rt-fMRI NF of a spatially circumscribed cognitive brain area - the aMCC. The aMCC, is part of the cingulate cortex, and has been categorized as a "limbic premotor cortex" with respect to its functions in integrating emotion, pain, cognitive control, and intentional motor control (Vogt, 2009; Shackman et al., 2011). The aMCC was chosen as a target region since the author's research group has had experience working on the cingulate cortex. The group previously investigated involvement of the ACC in the cognitive processes of anticipation and response conflict phases by using CPT and the Flanker task (Luetcke and Frahm, 2008; Luetcke et al., 2009). To address the open questions, this chapter first presents general challenges of rt-fMRI NF, and specific challenges of rt-fMRI NF of the aMCC; then proposes a novel NF paradigm as one possible solution to the challenges.

\subsubsection{Challenges of rt-fMRI NF}

\subsubsection{Technical and physiological aspects of feedback latency}

Most conventional rt-fMRI NF paradigms have been adapted from EEG NF paradigms. EEG's temporal resolution is in the order of milliseconds, which in the context of NF, allows short trials with immediate feedback, thus enabling subjects to try numerous strategies in one run. For example, in a EEG-NF study training of SCP, one trial lasts $8 \mathrm{~s}$, 120 trials were performed in a training session, and the EEG sampling rate was $250 \mathrm{~Hz}$ (Heinrich et al., 2004). On the other hand, fMRI has a relatively lower temporal resolution in the order of seconds. Temporal resolution of fMRI is determined by the TR (repetition time) which corresponds to the time to collect one brain volume consisting of multiple slices. In rt-fMRI NF, by applying gradient-echo echo planar imaging (EPI) sequences for imaging, TR is usually $2 \mathrm{~s}$, which translates to a sampling rate of $0.5 \mathrm{~Hz}-$ far lower than that of EEG. The frequency of updating the continuous feedback cannot be faster than the TR. There is also time needed for processing the data, which takes around 2 s. This means that, although the feedback in rt-fMRI NF can be presented continuously, the feedback is less instantaneous than in EEG NF.

Another important factor that adds to the delay of the feedback is the intrinsic physiological properties of the BOLD signal. The inherent temporal delay of approximately $6 \mathrm{~s}$, is because of the slow hemodynamic response to neuronal activation, and determines the delay between the onset of mental strategy (onset of neural activity) 
and reinforcement (feedback) (Weiskopf, 2012). Adding this factor to the time needed for acquisition and computation of the feedback signal, the "immediate" feedback subject sees at any moment actually reflects the mental strategy that happened $\sim 10 \mathrm{~s}$ earlier.

The nature of fMRI technique demands NF paradigms to be adapted to suit its temporal resolution. The average regulation block in rt-fMRI NF paradigm is a continuous 20-50-second block, during which the subjects are instructed to try out one strategy. Therefore, in rt-fMRI NF, there are fewer trials available in one run for subjects to try various strategies to regulate their brain activity.

\subsubsection{Psychological aspects}

By virtue of its design, the conventional rt-fMRI NF paradigm consequently brings about challenges on the psychological level in terms of implicit temporal contiguity and higher cognitive load.

\subsection{Temporal contiguity}

Temporal contiguity - the time interval between the response and the reinforcement - has been implicated as a factor that affects learning in NF (Sulzer et al., 2013). In a conventional rt-fMRI NF paradigm, subjects are normally instructed about the $\sim 8-10 \mathrm{~s}$ feedback delay, and in most studies, experimenters assume that subjects understand the temporal contiguity of the NF paradigm. In reality, subjects need to estimate the temporal contiguity of the feedback, and additionally need to associate the feedback with mental strategies used several seconds earlier, which could be challenging. Therefore, in a conventional NF paradigm, the temporal contiguity is implicit to the subjects; and not knowing the exact structure of temporal contiguity may hinder learning (Greville and Buehner, 2010).

\subsection{Cognitiveload}

The conventional way of doing rt-fMRI NF is by trying to self-regulate the BOLD brain activity using mental strategies, while simultaneously perceiving and appraising the continuous feedback of BOLD-activation from the target brain area. In a conventional rtfMRI NF paradigm, subjects have to find a strategy to self-regulate brain activity from a target ROI, pay visual attention to the feedback; and in parallel link the feedback to the strategy done earlier, and appraise the feedback. In most studies, experimenters assume that subjects are able to perform the task of self-regulation while associating the 
feedback with mental strategies that have been used several seconds earlier. This method of presenting feedback in parallel with performing the main NF task exerts a high cognitive load on the subjects, and may impair the outcome of rt-fMRI NF.

\subsubsection{Challenges of rt-fMRI NF of the aMCC}

In addition to the technical, physiological, and psychological challenges of the conventional NF paradigm, self-regulation of the aMCC itself presents its own unique challenges.

\subsubsection{Mental strategies}

The aMCC is a relatively complex brain area for which no specific mental strategies that can voluntarily and reliably activate it has been prescribed. Finding a cognitive control strategy to activate the aMCC is more difficult than finding a motor imagery strategy to self-regulate the somatomotor areas. This difficulty has been acknowledged by Mathiak and colleagues (Mathiak et al., 2010) who investigated a social NF paradigm that can circumvent the need for specific cognitive control strategies for NF of the ACC. By using social NF (in the form of smiling or frowning avatars) to increase motivation, the focus shifts on enhancing the value of reinforcements instead of the search for strategies.

\subsubsection{Regulating the cognitive control region}

Another difficulty in aMCC self-regulation is that the very area is also implicated in feedback-processing as part of its cognitive control function (Amiez et al., 2013). In the Parallel paradigm, this would add another layer of confound to the BOLD signal measured from the aMCC because it is hard to determine if the activity observed in the aMCC is due to self-regulation or feedback processing. Therefore, regulating an area that is part of the cognitive control machinery and that is involved in feedback processing is at best, difficult.

\subsubsection{Development of a novel rt-fMRI paradigm: the Serial paradigm}

Collectively, the difficulties of self-regulating the functions of aMCC with rt-fMRI NF may explain why rt-fMRI NF studies targeting the cingulate cortex including its various subdivisions are scarce (Weiskopf et al., 2003; deCharms et al., 2005; Mathiak et al., 2010; Hamilton et al., 2011; Gröne et al., 2014) compared to those targeting the motor cortex (DeCharms et al., 2004; Yoo et al., 2008; Auer and Frahm, 2011; Subramanian et al., 2011; Berman et al., 2012; Chiew et al., 2012; Johnson et al., 2012; Sitaram et al., 2012; Zhang et al., 2014). 
Addressing the described challenges in rt-fMRI NF and self-regulating the aMCC was the motivation behind developing a paradigm that would make self-regulation of a cognitive area more attainable to the subjects. This study introduces a novel rt-fMRI NF paradigm: the "Serial" paradigm which uncouples the self-regulation phase from the feedback appraisal phase. In the Serial paradigm, feedback is presented only after, and non-concurrent with the self-regulation phase. This reduces the processing of the cognitive load from parallel to serial, and thus reduces the burden in performing the main NF self-regulation task, unlike in the conventional "Parallel" paradigm. In addition, the Serial paradigm has an explicit temporal contiguity structure. Most importantly, the Serial paradigm, enables subjects to process self-regulation and feedback appraisal separately, and thus reduces difficulties in regulating a region which is itself involved in feedback appraisal. This study hypothesizes that the Serial paradigm would reduce interference between self-regulation and feedback appraisal, and thus would help achieve success in self-regulation of the aMCC.

One similar study that specifically examined delayed, non-concurrent feedback presentation (Johnson et al., 2012) only came to the author's knowledge while finishing the study. In that pilot study, the conventional continuous feedback presentation was compared to an intermittent feedback presentation. Intermittent presentation of feedback (about $20 \mathrm{~s}$ delay) was found to be more effective than continuous presentation in a motor imagery task (Johnson et al., 2012).

\subsubsection{Aims and goals}

The goal of this exploratory study was to investigate the possibility of learned selfregulation of a circumscribed brain area involved in cognitive control, the aMCC, through extensive rt-fMRI NF training. To meet the challenges associated with rt-fMRI NF of the aMCC, a novel Serial paradigm was introduced and compared to the conventional Parallel paradigm. To study the neural correlates associated with the two different paradigms, and with the learning processes in rt-fMRI NF; aMCC BOLD-activity was monitored during NF training in the presence of feedback, and during the transfer session (performed pre- and post-training), in the absence of feedback. This study also aims to investigate brain activity beyond the aMCC, hence whole-brain activation during the transfer session was investigated. This study also explores the neural correlates of feedback appraisal. Questionnaires explored the applied mental strategies as well as the perceived difficulty and performance in the two NF paradigms. 


\subsection{Material and methods}

\subsubsection{Participants}

Thirty healthy, non-smoking, right-handed subjects (12 males, mean age $27.4 \pm 2.6$ years) participated in the study. All subjects provided written informed consent and received compensation for their participation. The study was approved by the local ethics committee of Georg-Ellias-Müller-Institute for Psychology at University of Göttingen, Germany. Twenty subjects were assigned to undergo NF training, and were randomly, and equally assigned to the Parallel Training (PT) or the Serial Training (ST) group. Ten age-matched subjects were assigned to the Control group which did not undergo NF training, and were further equally assigned into Parallel Control group (PC) or Serial Control group (SC).

\subsubsection{Experimental procedure overview}

Subjects underwent 10 MRI sessions: one initial, one pre-training, six NF trainings, one post-training, and one final session (Figure 7A). The initial and the final session consisted of a high-resolution anatomical MRI, diffusion-weighted MRI, and a restingstate fMRI measurement. The initial session also was also performed to familiarize subjects with the scanning environment. In the pre-training session, subjects first underwent one fMRI run performing the CPT, one run performing a Flanker task and one run performing the assigned NF paradigm without receiving feedback. Before subjects entered the scanner they underwent a short demonstration of all tasks to assure full understanding.

The following six NF training sessions were distributed on alternate days (Monday, Wednesday, Friday) for two weeks. In each NF training session, subjects underwent three runs performing the assigned NF training protocol and received feedback. Two days after the last training session, subjects underwent a post-training session with the same measurements as in the pre-training session, but in reverse order. Control subjects underwent the initial, the pre-training, the post-training and the final session. Pre- and post-training sessions were separated by a two-week period without NF training.

\subsubsection{MRI data acquisition}

All MR images were acquired on a 3T Tim Trio MRI scanner (Siemens Healthcare, Erlangen, Germany) using a 32-channel head coil for signal reception. Structural wholebrain T1-weighted images were obtained by an inversion-recovery 3D FLASH sequence 
$\left(\mathrm{TR}=2530 \mathrm{~ms}, \mathrm{TE}=3.26 \mathrm{~ms}\right.$, flip angle $=7^{\circ}, \mathrm{TI}=1100 \mathrm{~ms}, 192$ slices per slab $)$ at $1.0 \times$ $1.0 \times 1.0 \mathrm{~mm}^{3}$ isotropic resolution. All BOLD fMRI measurements were obtained by a gradient-echo $\mathrm{EPI}$ sequence $\left(\mathrm{TR}=2 \mathrm{~s}, \mathrm{TE}=36 \mathrm{~ms}\right.$, flip angle $=70^{\circ}$, acquisition matrix $=$ $96 \times 96$ ) at $2.0 \times 2.0 \times 4.0 \mathrm{~mm}^{3}$ resolution with 22 slices oriented along the AC-PC line, encompassing the cerebrum until the mid-brain. Individual slice positions from the first fMRI scanning session were saved and subsequently re-applied in all sessions to minimize slice positioning differences between datasets. Motion correction on fMRI data was performed in k-space (online software of the manufacturer). To optimize registration of the partial-brain fMRI scan to the structural whole-brain MRI, a single whole-brain EPI measurement $\left(\mathrm{TR}=3250 \mathrm{~ms}, \mathrm{TE}=36 \mathrm{~ms}\right.$, flip angle $=70^{\circ}, 36$ slices $)$ was obtained in each subject at the same resolution as in the BOLD fMRI.

\subsubsection{ROI selection}

In the pre-training session, the aMCC was individually defined based on the fMRI analysis of the CPT, as described in Section 2.2.

\subsubsection{Neurofeedlback training paradigms}

The Parallel paradigm started with an initial baseline period of $50 \mathrm{~s}$, followed by six cycles of a $20 \mathrm{~s}$ "Think" phase alternating with a $30 \mathrm{~s}$ "Count" phase, and ended with a 20 $\mathrm{s}$ baseline period (Figure 7B). One Parallel paradigm run lasted $6.17 \mathrm{~min}$ (185 fMRI images). The Serial paradigm, which uncouples the self-regulation phase and the feedback appraisal phase, started with an initial baseline period of $40 \mathrm{~s}$, followed by 8 cycles of $10 \mathrm{~s}$ "Think" phase, $10 \mathrm{~s}$ "Feedback Think", $10 \mathrm{~s}$ "Count", $10 \mathrm{~s}$ "Feedback Count" and ended with a $10 \mathrm{~s}$ baseline period (Figure 7C). One Serial paradigm run also lasted $6.17 \mathrm{~min}$ (185 fMRI images).

Visual feedback in the form of a vertical fluctuating thermometer scale, which had 21 gradations of color from blue (low) to red (high), was presented on a projection screen in the scanner bore. All stimuli were shown on a white background. The feedback in the Parallel and Serial paradigm was updated every $2 \mathrm{~s}$ (once per TR). Subjects in the PT group received continuous feedback and were informed about its intrinsic delay of 810 seconds (hemodynamic delay plus image acquisition plus rt-fMRI processing time). This information was not critical for the ST group since they received the feedback during the "Feedback" phase after each "Think" or "Count" phase. 
All subjects in the NF training group were instructed to try to self-regulate the BOLD-activation in the individual aMCC ROI. They were instructed to develop mental strategies that result in an increase of the feedback signal, i.e. increase in the level of the thermometer bar during the up-regulation "Think" phase. They were also instructed to decrease the feedback signal, i.e. to decrease the level of the thermometer during the down-regulation "Count" phase by either counting backwards or finding a mental strategy with an equivalent effect. The ST group was additionally instructed to just perceive the feedback during the "Feedback" phase.

Subjects were given suggestions about cognitive strategies that might help them influence self-regulation of the aMCC. In line with Shackman's proposal about aMCC function (Shackman et al., 2011), the suggested strategies were in the domain of cognitive control (e.g. making plans and decisions) and emotion (e.g. think about a negative situation). Subjects were encouraged to try various strategies and to find what works best for them in the NF training runs and were suggested to keep a strategy constant within one phase.

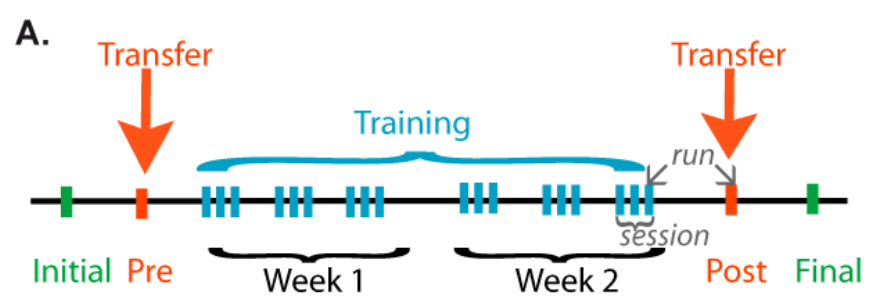

B.

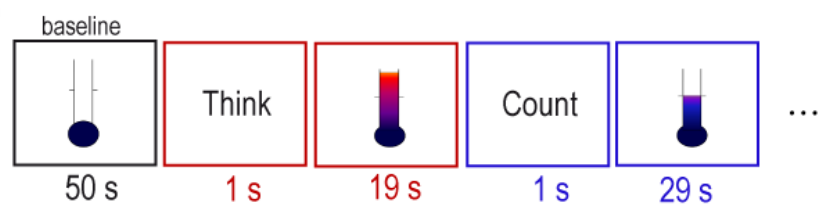

C.

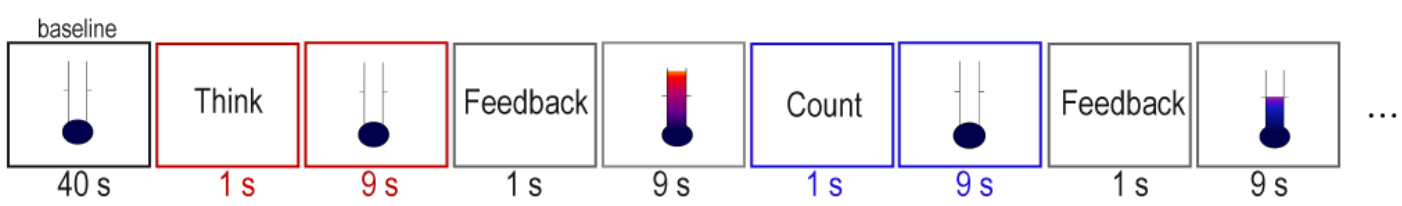

Figure 7: A. Structure of the overall neurofeedback sessions. B. Example stimuli of the Parallel paradigm and the $\mathbf{C}$. Serial paradigm

\subsubsection{Real-time fMRI neurofeedback}

A custom in-house rt-fMRI NF system for rt-fMRI analysis and feedback presentation developed by Tibor Auer and Henry Luetcke in MATLAB (The MathWorks, Inc., USA) was integrated in the MRI scanner computer system (Dewiputri and Auer, 2013). Realtime data export from the MRI scanner via FTP allowed online fMRI analysis. Motion 
correction with SPM Realign algorithm (http://www.fil.ion.ucl.ac.uk/spm/) was performed as a pre-processing step.

The reference ROI was a large background region (a 22-slices brain volume as in the BOLD fMRI measurements) and it was used to cancel out any global unspecific BOLD changes e.g. general changes in blood flow, respiration etc. For both the aMCC target ROI and the background reference ROI, a normalized BOLD signal was calculated for each time point with reference to the average of 5 time points of the preceding "FeedbackCOUNT" phase in the Serial, and 10 time points of the preceding "COUNT" phase in the Parallel paradigm respectively. The feedback signal (FS) given to the subjects was the difference between the normalized BOLD signal of the aMCC and the background, calculated as:

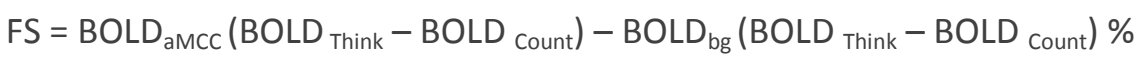

The following descriptions were the steps taken to relate FS to the thermometer scale. To minimize the sensitivity of the normalized BOLD to signal fluctuations around zero, a double logistic-like function for calculating the FS with the following characteristics was implemented: a range from 0 to 21 of the thermometer gradations (corresponding to $-2 \%$ to $+2 \%$ signal change respectively), and a flat center between $0.25 \%$ and $+0.25 \%$ signal change.

\subsubsection{Pre-Post measurements}

All subjects performed the self-regulation task without feedback in the pre-training and the post-training run, termed the "transfer" condition. An empty thermometer was shown in place of the feedback. The transfer condition is to test how well the subjects were able to self-regulate the aMCC in the absence of a feedback.

\subsubsection{Questionnaires}

Questionnaires were administered to the subjects outside the scanner before and after each scanning session. Before each session, subjects rated their valence and arousal using a 5-point visual scale, which was adapted from the Self-Assessment Manikin (SAM) (Bradley and Lang, 1994). In addition, in the pre-training session, subjects also answered a question about experience in meditation. After each session, subjects rated the difficulty and perception of their own performance of the self-regulation phases; their valence, arousal and dominance (adapted from the SAM); and described their mental strategies during the self-regulation phases. Examples of the questionnaire can 
be found in the Appendix. Wilcoxon Signed Ranks Test was performed on the subjects self-ratings using SPSS Version 21 (IBM, USA).

\subsubsection{Offline fMRI data analyses}

\subsubsection{ROI analyses}

The fMRI signal time-courses from the NF training runs and transfer runs were extracted from the aMCC and the background region for offline ROI analysis using a custom in-house NF toolbox implemented in MATLAB.

To describe changes in aMCC activity of self-regulation, GLM was performed on the time courses extracted from individual ROIs, with the following regressors: "Think" phase in the Parallel paradigm; or "Think" and "Count" phases in the Serial paradigm. Parameter estimates (converted to Percent Signal Change) for the aMCC (PSC $\mathrm{AMCC}_{\text {) }}$ and the background region ( PSC $_{\text {bg }}$ ) were computed. The GLM contrast was Think > Count.

In the Transfer condition, PSC of the BOLD response from the aMCC was analyzed by applying a 3-way mixed ANOVA with factors: PARADIGM (Serial vs. Parallel), GROUP (Training vs. Control) and Time (Post vs. Pre).

To assess individual transfer success and to subsequently classify successful individuals as Learners, the difference in PSC between post- and pre- training was averaged: the values from the Serial control group and Parallel control group served as thresholds to define Learners in Serial training and Parallel training group respectively. Learners were defined as individuals who had higher change in aMCC activity after NFtraining compared to the average control group. Association between learning status and paradigm was tested using Fisher's Exact Test.

Subsequent analyses explored the difference between Learners and NonLearners, independent of the Serial or Parallel training paradigm, i.e. the Learners from the ST group and PT group were combined and compared with the Non-Learners across both training paradigms. In the transfer condition, PSC of the BOLD response from the aMCC was analyzed by applying a 2-way mixed ANOVA with factors: LEARNING STATUS (Learner vs. Non-Learner), and Time (Pre vs. Post). In the Training condition, PSC of the BOLD response from the aMCC was analyzed by applying a 2-way mixed ANOVA with factors: PARADIGM (Serial vs. Parallel), Time (Training 1...Training 18).

On the level of the individual subjects, the PSC of the aMCC of each of the 18 training runs was normalized to the PSC of the pre-training measurement PSC $_{\text {train }}$ - 
$\mathrm{PSC}_{\text {pre }}$ ) and then thresholded using the average signal difference ( $\mathrm{PSC}_{\text {post }}$ - $\mathrm{PSC}_{\text {pre }}$ ) of the Serial or Parallel paradigm control group. Trainings with a higher increase than the appropriate control group were defined as efficient training runs (ETR) and summed up for each subject. ETR was compared between learning statuses using Student's T-test. The correlation between ETR and Post-Pre PSC group difference was determined using Pearson's correlation coefficient.

The uncoupling of the self-regulation phase from the feedback appraisal phase in the Serial paradigm enables a separate analysis on the feedback appraisal phase. GLM was performed on the time courses extracted from individual ROIs, with regressors modelling "Feedback" and "Count" phases. The GLM contrast was Feedback > Count. BOLD activity of aMCC during feedback appraisal was investigated throughout the Training runs in Learners $(n=6)$ and in Non-Learners $(n=4)$ from the ST group. PSC of the BOLD response from aMCC was analyzed using a 2-way repeated-measures withinsubject ANOVA with factors: TASK (Self-regulation vs. Feedback appraisal) and TIME (Training 1...Training 18).

\subsubsection{Whole-brain fMRI analyses}

Additional to the ROI analyses, voxel-wise fMRI analysis of the whole volume covering the cerebral cortex was performed using standard block design GLM in FSL (www.fmrib.ox.ac.uk/fsl). Pre-processing steps involved motion correction using MCFLIRT (Jenkinson et al., 2002); non-brain removal using BET (Smith, 2002); spatial smoothing using a Gaussian kernel of FWHM 5 mm; grand-mean intensity normalization of the entire 4D dataset by a single multiplicative factor; and high-pass temporal filtering (Gaussian-weighted least-squares straight line fitting, with sigma $=15.0 \mathrm{~s}$ ). Registration using linear rigid body registration (FLIRT) with 6 degrees of freedom was performed to register the partial fMRI volume to the whole-brain volume, and further refined using boundary-based registration (BBR) to register whole brain volume to the anatomical T1-weighted image (Jenkinson and Smith, 2001; Jenkinson et al., 2002). Registration was then further refined using non-linear registration (FNIRT) on the anatomical T1weighted image to the standard MNI space (Andersson et al., 2007).

First-level GLM was performed on the fMRI data with the following regressors: "Think" phase for self-regulation in the Parallel paradigm; "Think" and "Count" phases for self-regulation in the Serial paradigm; "Feedback" and "Count" phases for feedback appraisal in the Serial paradigm. In the higher-level analysis, mixed effects modeling in 
FSL (FLAME $1+2$ ) was used. The higher level model is specified in the following two sections (3.2.9.2.1 and 3.2.9.2.2). $Z$ (Gaussianized T) statistic images were thresholded using clusters using $Z>3.1$ and a cluster significance threshold of $\mathrm{p}=0.05 \mathrm{FWE}$ corrected for multiple comparisons. Brain regions that are activated are labeled according to the Harvard-Oxford cortical and sub-cortical structural probabilistic atlas.

\subsection{Self-regulation in Learners versus Non-Learners}

The Learners from the ST group $(n=6)$ and PT group $(n=2)$ were combined. The four non-learners from the ST group were re-grouped with randomly chosen four subjects from the PT group as "Non-Learners". Four subjects from the SC and PC group were randomly selected and re-grouped as "Controls". Therefore, the total number of subject in each group was eight. For the Transfer condition, changes in brain activity in Learners, Non-Learners and Control were investigated by 2-way mixed ANOVA on the first level GLM contrast Think > Count with factors TIME (Pre vs. Post) and LEARning STATUS (Learner vs. Non-Learner, Learner vs. Control, and Non-Learner vs. Control).

\subsection{Group analysis of feedback appraisal in Serial paradigm}

Analysis on feedback appraisal was performed on subjects of the Serial paradigm, (ST group, $\mathrm{n}=10$ ). Initial analysis on changes in feedback appraisal activity was done by a paired t-test on the first level GLM contrast Feedback > Count, comparing between the last training run (Training 18) and the first training run (Training 1). Subsequent post$h o c$ analysis focused on exploring the brain activity during feedback appraisal in the first and the last training run separately. For this, a one-sample t-test on the first level GLM contrast Feedback > Count was performed on each run.

\subsection{Results}

\subsection{1 aMCC ROI analysis}

\subsubsection{Transfer of self-regulation: group analysis}

Success in learning to self-regulate the aMCC was based on the pre- to post-training measurement difference in aMCC BOLD activity levels during self-regulation without feedback (transfer condition) compared to the same measurements in the no-training control groups.

Group performance (PSC) of the four groups in self-regulation in the Transfer condition is shown in Figure 8. The 3-way ANOVA (PARAdigm, Group, TimE) revealed no 
significant main effects in GROUP or TIME, as well as no significant interactions between these main factors. This reflects that there was no difference in the aMCC BOLD activity either between the trained group and the untrained control group, or between the preand the post-training transfer runs. The lack of significant interaction between PARADIGM $\times$ GROUP $\times$ TIME shows that there was no significant difference in aMCC BOLD activation from pre- to post-training neither between the trained and the untrained control group, nor in the trained group between the Parallel and the Serial paradigm. On the group level, there was no significantly larger increase from pre- to post-training transfer condition in the trained group compared to the untrained control group.

However, there was a main effect of PARADIGM in which both Trained (ST) and Controls (SC) of the Serial paradigm showed higher aMCC BOLD activity than the Trained $(\mathrm{PT})$ and Controls $(\mathrm{PC})$ of the Parallel paradigm $\left(\mathrm{F}_{1,26}=8.036, \mathrm{p}=0.009\right)$.

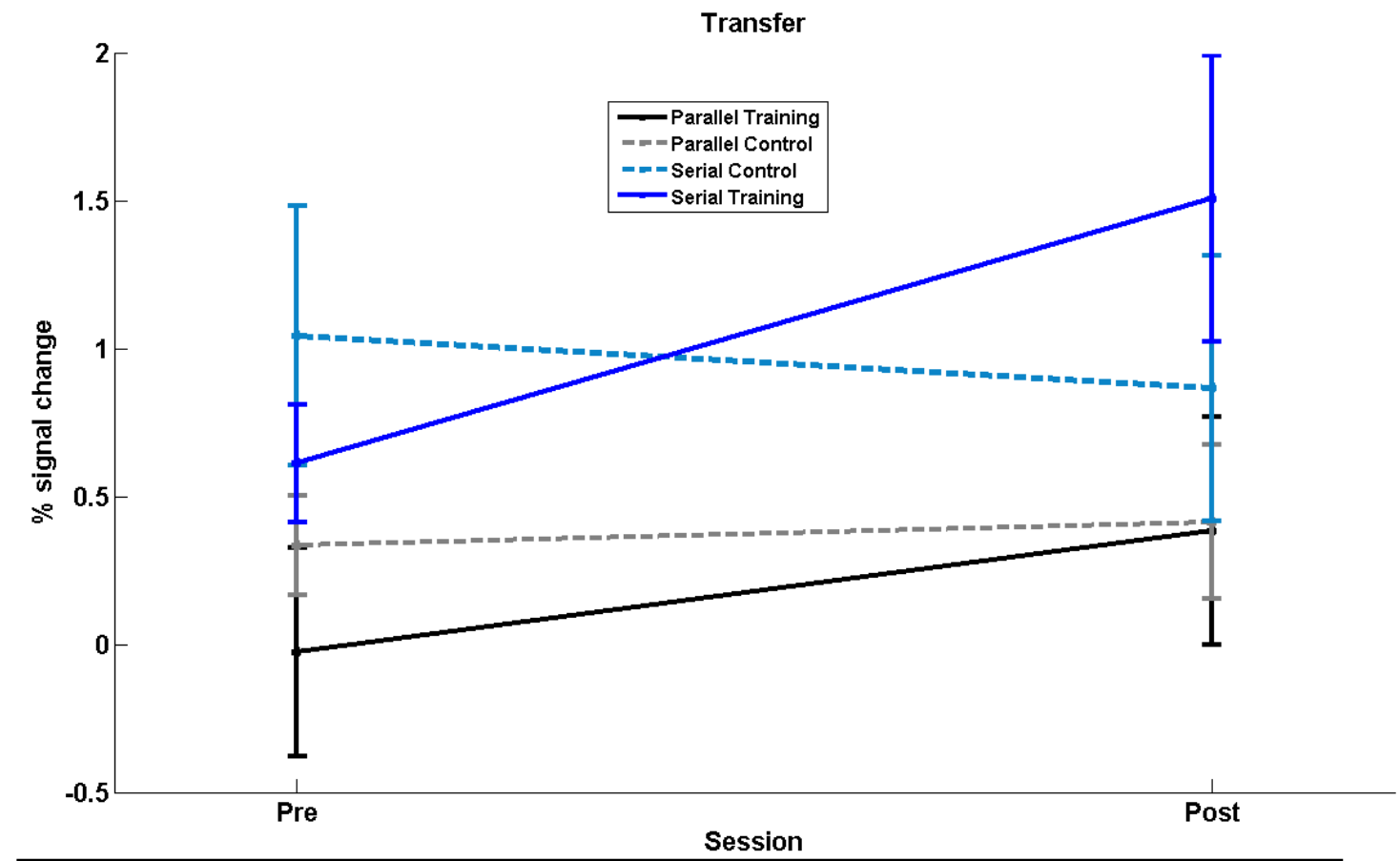

Figure 8: Group performance in aMCC self-regulation in the transfer session (group mean \pm SEM)

\subsubsection{Transfer of self-regulation: individual analysis}

Individual performances (PSC) in the pre- and post-training transfer run were investigated (Figure 9) to gain insight into possible explanations for the lack of a significant increase in group aMCC activity after the rt-fMRI NF training. These performances reveal large individual difference in aMCC BOLD activity between the two measurements and led to a categorization of the individual subjects into Learners and 
Non-Learners. Learners were defined as individuals with an above-threshold PSC difference of the aMCC BOLD activity from the pre- to post-training transfer run. The threshold value was calculated for each paradigm from the equivalent untrained control group (see description in Section 3.2.9.1). Based on this categorization, more learners were observed in the Serial Training group (6 out of 10) than in the Parallel Training group (2 out of 10). However, this difference in number of Learners in the different paradigms did not reach significance (Fisher's Exact Test, $\mathrm{p}=0.170$ ). 
A.
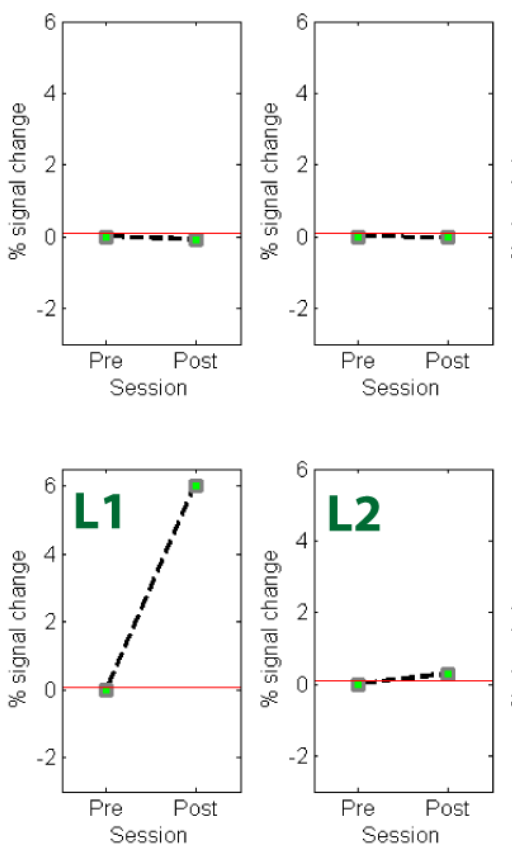

Parallel Paradigm transfer
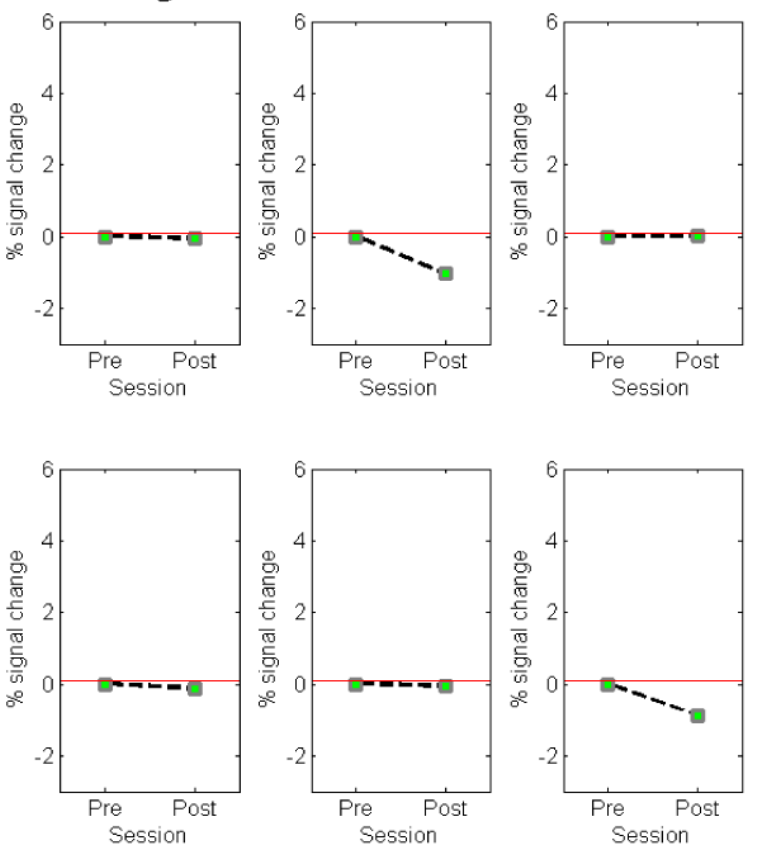

B.
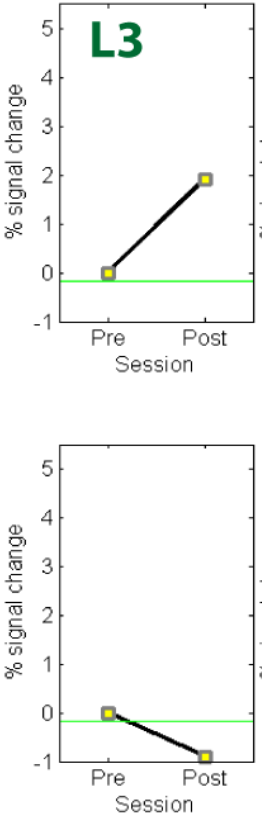

Serial Paradigm Transfer
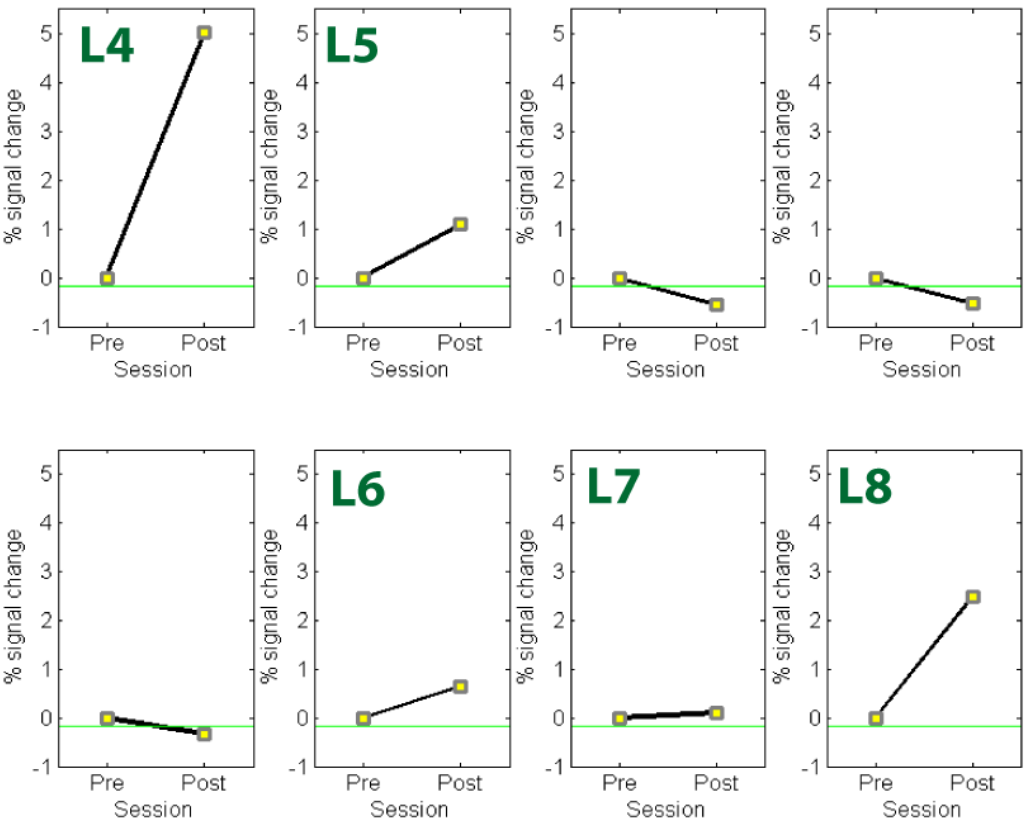

Figure 9: Individual performance in NF transfer session in subjects from A) Parallel Training group B) Serial Training group. Threshold represents the average difference from the Pre- and Post transfer measurements of the two untrained control groups: Parallel Control (red line), Serial Control (green line). Subjects whose post-pre difference in PSC was higher than the threshold were classified as learners $(L)$ and are numbered. 


\subsubsection{Transfer of self-regulation: group analysis of learning status}

Subjects were re-grouped (Section 3.2.9.1) based on learning status independent of the $\mathrm{NF}$ paradigm, and the group performance in self-regulation in the transfer condition is shown in Figure 10. The 2-way ANOVA (LEARNing, TIME STATUS) showed a significant main effect of TIME, $\left(\mathrm{F}_{1,18}=7.98, \mathrm{p}=0.011\right)$, and a significant TIME $\times$ LEARNING STATUS interaction $\left(\mathrm{F}_{1,18}=15.83, \mathrm{p}=0.001\right)$, where the Learners showed significant increase in aMCC BOLD activity after NF training $\left(\mathrm{t}_{7}=-2.802, \mathrm{p}=0.026\right)$, whilst the Non-Learners showed a significant decrease $\left(t_{11}=3.316, p=0.0069\right)$ (Figure 10). Comparing the two learning statuses at post-training session, the Learners showed significantly higher aMCC activity than non-learners ( $\left.\mathrm{t}_{18}=-3.54, \mathrm{p}=0.017\right)$.

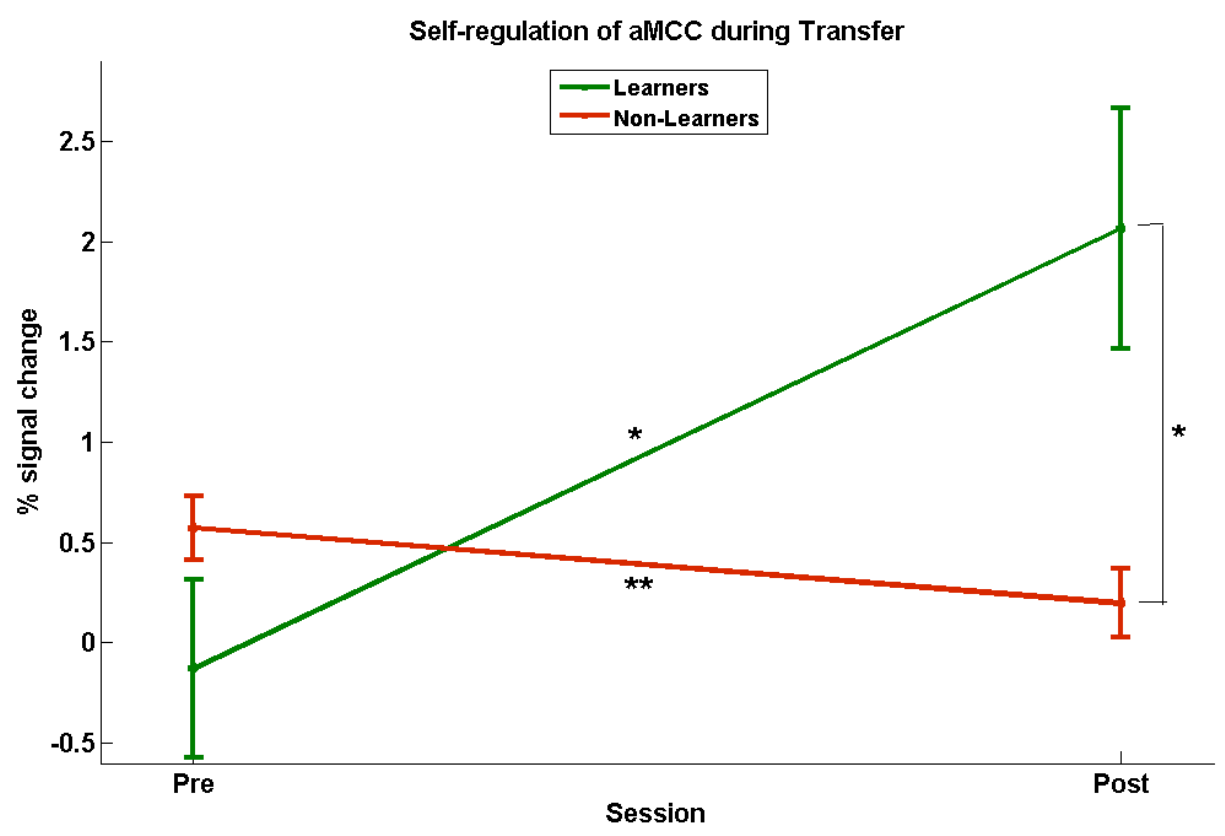

Figure 10: Performance of Learners $(n=8)$ and Non-Learners $(n=12)$ in aMCC selfregulation in the transfer session (group mean \pm SEM). Significant post-hoc t-tests are marked with asterisk $(* p<0.05 ; * * p 0.01)$

\subsubsection{Training of self-regulation: group analysis of paradigms}

Group performance (PSC) of the two trained groups in self-regulation in the Training condition is shown in Figure 11. The 2-way ANOVA (TIME, PARADIgm) showed no significant main effect of TIME, indicating no change of aMCC BOLD activity throughout the training; but a highly significant main effect of PARADIGM $\left(F_{1,18}=9.237, p=0.007\right)$ where the Serial Training group showed higher aMCC BOLD activity throughout the NF Training compared to the Parallel Training group. There was no significant interaction between the two factors. 


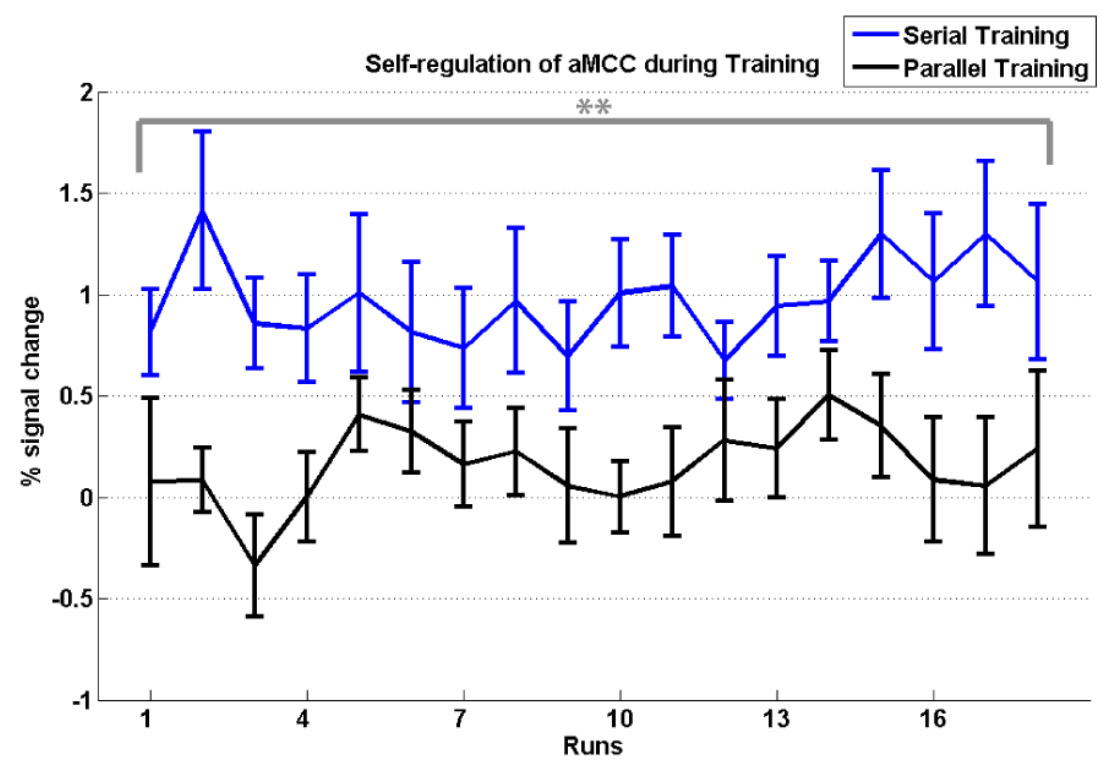

Figure 11: Group performance of aMCC self-regulation Serial and Parallel Training group during the two-week training sessions (group mean \pm SEM). ${ }^{* *} p<0.01$ for the main effect PARADIGM

\subsubsection{Training of self-regulation: individual analysis}

Similarly as in the Transfer run, the aMCC BOLD activity over the course of NF training was also investigated in individuals. Subjects did show large individual variability in the temporal patters of changes in aMCC activity throughout the 18 NF training runs (Figure 12). Even in Learners, there was no generalizable pattern of increase of aMCC activity over time that could distinguish them from the Non-Learners. A test considering the simplest temporal learning pattern, a linear increase in aMCC BOLD activity over the time course of the training, showed that five out of eight Learners and five out of twelve Non-Learners did show this linear increase in aMCC BOLD activity over time. No significant association could be found between a linear increase and learning status (Fisher's Exact Test, $\mathrm{p}=0.53$ ).

Since no consistent temporal pattern of changes in aMCC BOLD activity across the training could be observed, the absolute number of runs with increased aMCC BOLD activity was considered. These efficient training runs (ETRs) showed larger change of aMCC BOLD activity from the pre - to the post-training transfer run than observed in the untrained controls (Section 3.2.9.1). Summing up the numbers of ETR in each subject revealed that the Learners had an average of 13 ETRs (SEM = 2.3) out of 18 training runs, compared to the Non-Learners having on average of 6 ETRs (SEM $=1.4$ ). This difference in the number of ETRs between Learners and Non-Learners was significant $\left(t_{18}=-2.774, \mathrm{p}=0.013\right)$. 
A.

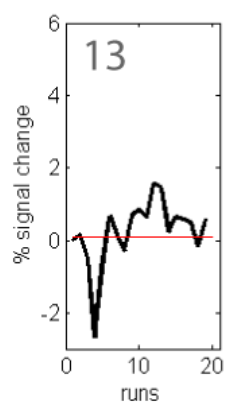

Parallel paradigm Training
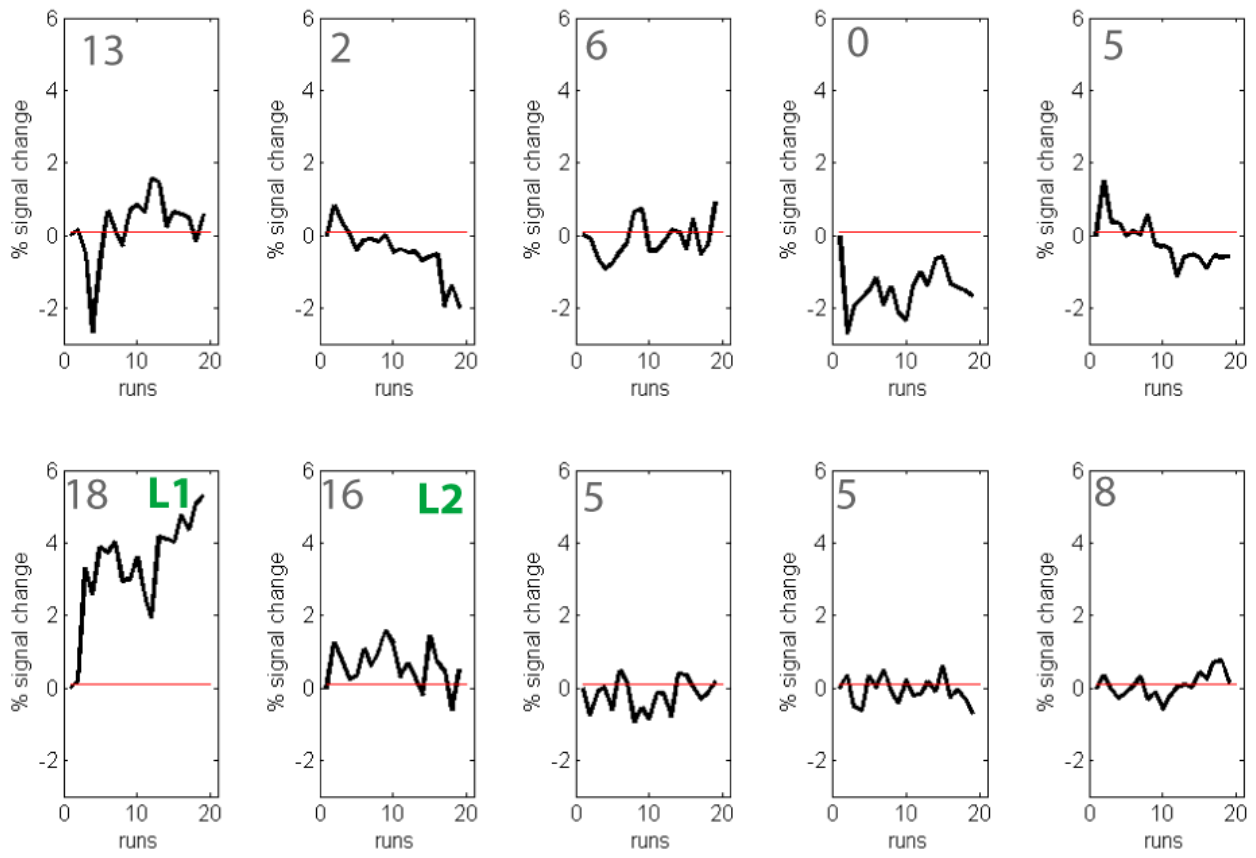

B.
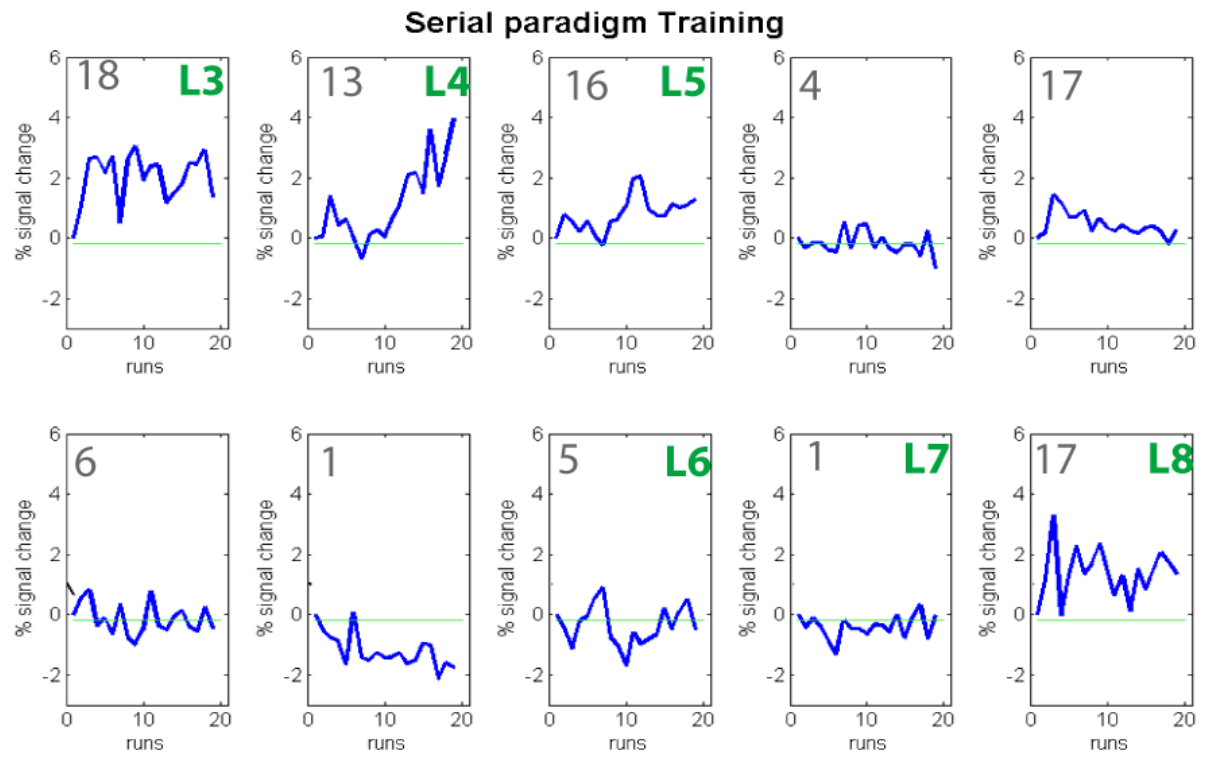

Figure 12: Individual performance in aMCC self-regulation during the training sessions in A) PT group and B) ST group. The number of efficient training runs (ETR) is shown in the upper left corner. Training efficiency was determined by the total number of training runs whose PSC exceeds a threshold calculated from the respective control group. Learners, defined from NF transfer condition (see text) are labelled "L" and are numbered.

\subsubsection{Training of self-regulation: group analysis of learning status}

Group performance (PSC) of the Learners and Non-Learners in self-regulation across the training condition is shown in Figure 13. The 2-way ANOVA (Learning Status, Time) 
revealed a significant main effect of LEARNING STATUS ( $\left.F_{1,18}=5.810, p=0.027\right)$, where the Learners, on average showed higher BOLD activity in the aMCC throughout NF training compared to the Non-Learners. The main effect of Time did not reach significance, as the BOLD activity did not significantly increase throughout the time course of the training. The interaction between Time and LEARning STATUS, $\left(F_{5.5,95.2}=1.97, p=0.086\right)$ also did not reach significance as would have been expected. The level of PSC within the Learners rose early in the training above the PSC level of the Non-Learners, but only in the last six training runs did a difference evolve. Post-hoc t-test revealed a significant difference in aMCC activity between Learners and Non-Learners $\left(\mathrm{t}_{18}=-3.050, \mathrm{p}=0.007\right)$ in the last training run.

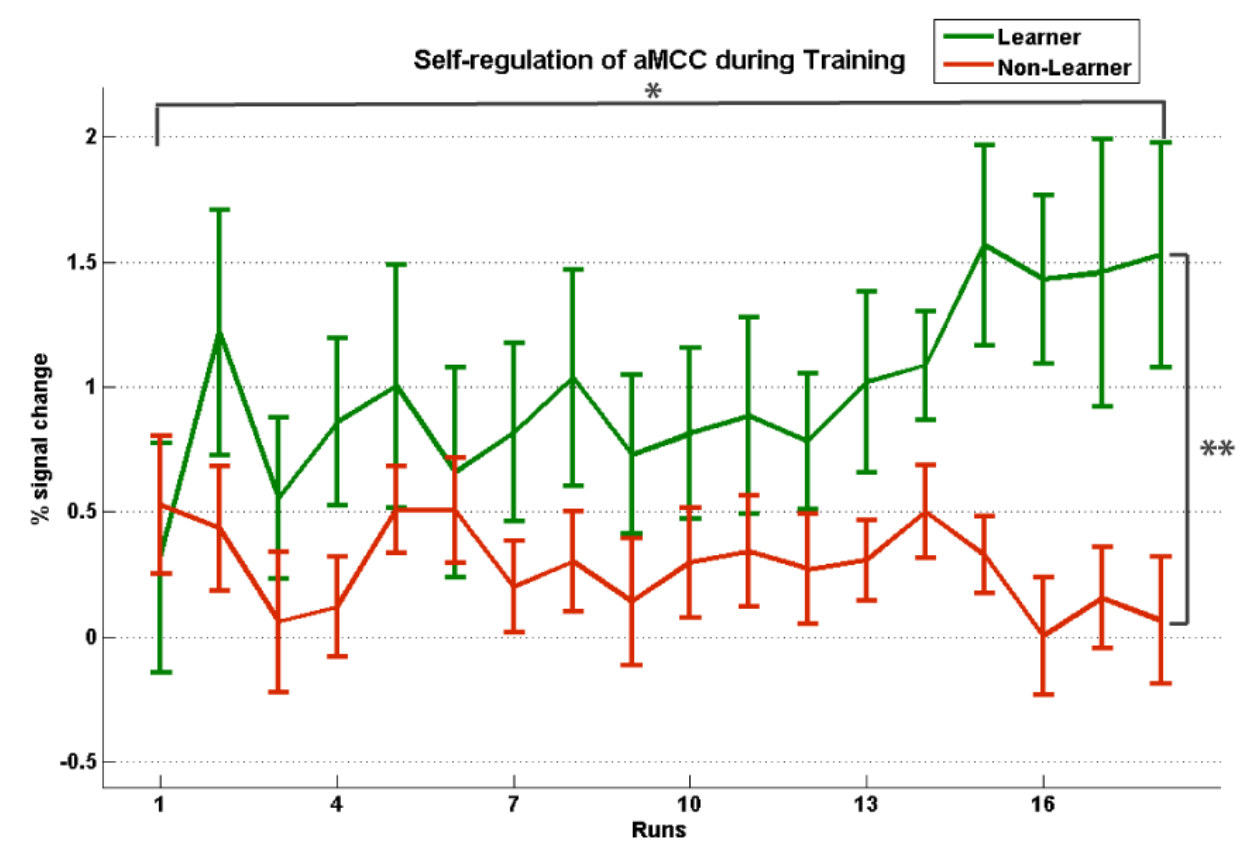

Figure 13: Group performance of aMCC self-regulation in Learners and NonLearners during the two-week training sessions (group mean \pm SEM). (*p $<0.05$ for main effect of LEARNING STATUS; $* * p<0.01$ for post-hoc t-test for last training run)

\subsubsection{Correlation between transfer success and Efficient Training Runs}

The relationship between efficiency in the training runs and success in transfer condition can be seen in Figure 14. The correlation between the number of ETR and transfer success was $r=0.60$ for the Serial Paradigm, and $r=0.67$ for the Parallel paradigm. In general, higher number of ETR was associated with higher post-pre PSC difference. However, there were subjects who had high number of ETR in the training condition, but did not somehow show a large post-pre difference in BOLD aMCC activity 
in the transfer condition. Alternatively, there were subjects who had a moderate number of ETR, yet succeeded in the transfer condition (for example, individual L6).

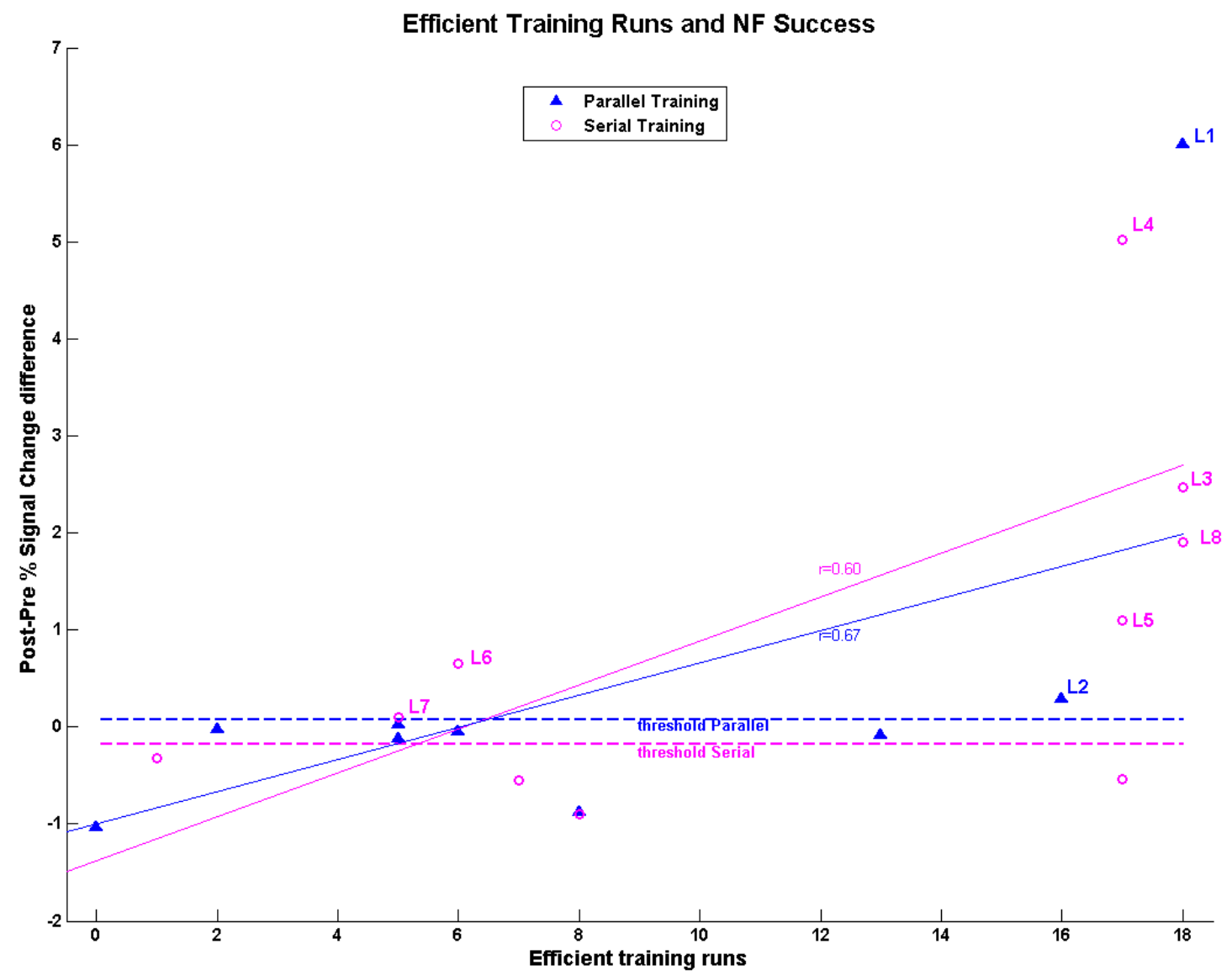

Figure 14: Relationship between number of efficient training runs (ETR) and individual training success. Least-squares lines and correlation coefficients are shown. Learners are labeled according to Figure 9. Dashed lines are thresholds calculated from the respective control groups.

\subsubsection{Feedback appraisal: group analysis}

The temporal uncoupling of self-regulation and feedback appraisal in the Serial paradigm enables separate investigation into feedback appraisal. The 2-way ANOVA (TASK, TIME) showed a significant main effect of TASK $\left(F_{1,9}=16.93, p=0.003\right)$, where aMCC BOLD-activity was lower during feedback appraisal than during self-regulation regardless of the course of NF training (Figure 15 A). There was no significant main effect of TIME; indicating that regardless of self-regulation or feedback appraisal that was being performed, there was no significant change in aMCC activity throughout NF training. In addition, there was also no significant TASK $\times$ TIME interaction, indicating that the change in aMCC activity across time, when the subjects performed self-regulation was not different from when they were appraising feedback. 
When the group of trained subjects was regrouped according to the learning status, 2way ANOVA (LEARNING STATUS, TIME) revealed a non-significant main effect of LEARNING STATUS, indicating that feedback appraisal elicited comparable aMCC BOLD-activity in Learners and Non-Learners (Figure 15 B,C).

A.

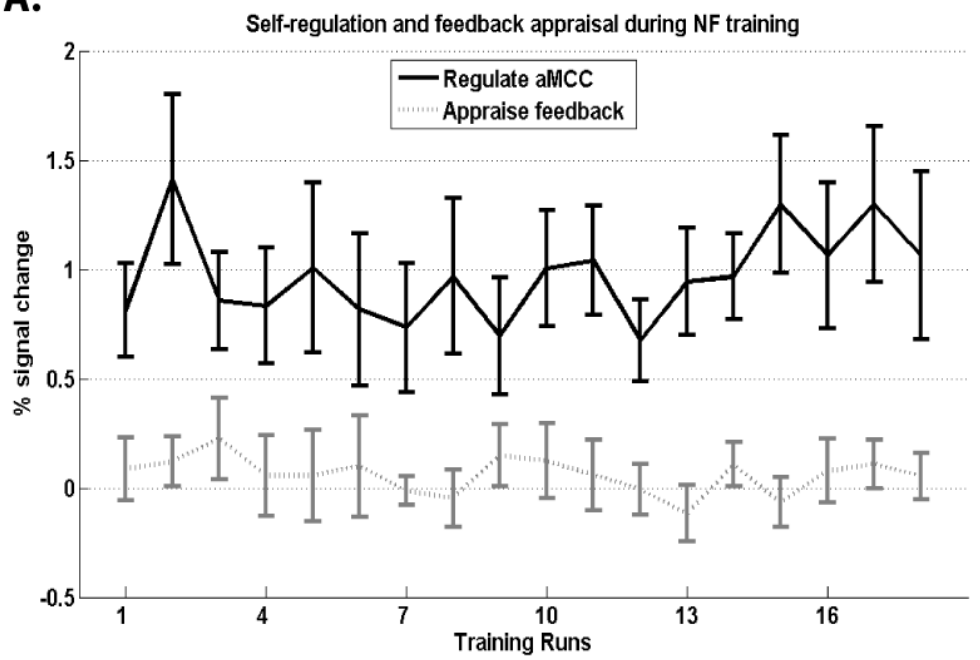

B.

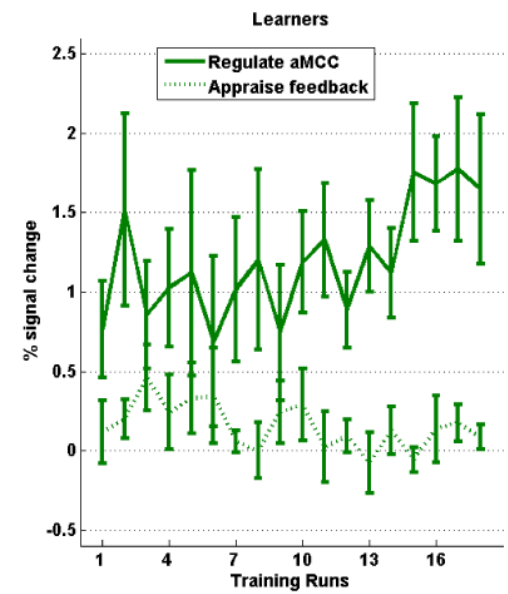

C.

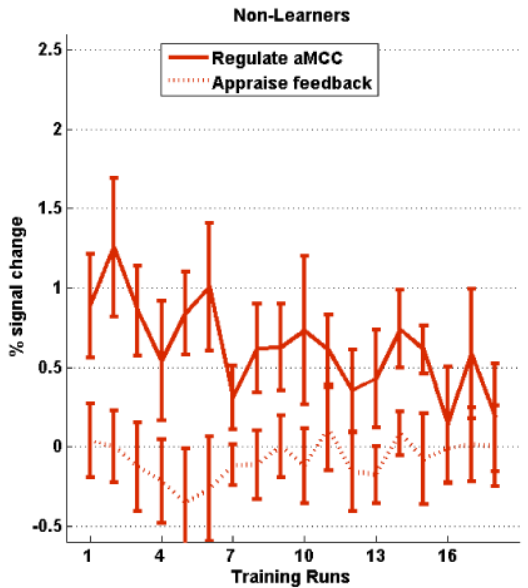

Figure 15: aMCC activity (mean \pm SEM) of self-regulation and feedback appraisal during NF training in A) Serial Training group and further divided into B) Learners C) Non-Learners

\subsubsection{Whole-brain analyses}

\subsubsection{Transfer of self-regulation: group analysis}

Whole-brain group level analyses, comparing between Learners, Non-Learners and Controls were performed to explore activations in brain regions other than, or in addition to the trained ROI. Brain regions activated during aMCC self-regulation higher in one group than in the other, in the post-training transfer compared to the pre-training transfer are shown in Figure 16. 
Compared to the Controls, the Learners showed increased activity mainly in the aMCC, thalamus, basal ganglia, SMA, and ventromedial prefrontal cortex (Figure 16 A, Table 1A). The Non-Learners, compared to Controls, showed increased activity mainly in the precuneus, the posterior cingulate cortex (PCC), and the inferior parietal lobule (IPL) (Figure $16 \mathrm{~B}$, Table 1B).

When comparing Learners and Non-Learners, increased activity in the aMCC, thalamus, basal ganglia and SMA - similar brain areas as in the Learner > Control comparison, could be seen. In addition to these areas, compared to the Non-Learners, the Learners had also increased left inferior frontal gyrus and left insula (Figure 16 C, Table $1 \mathrm{C}$ ).
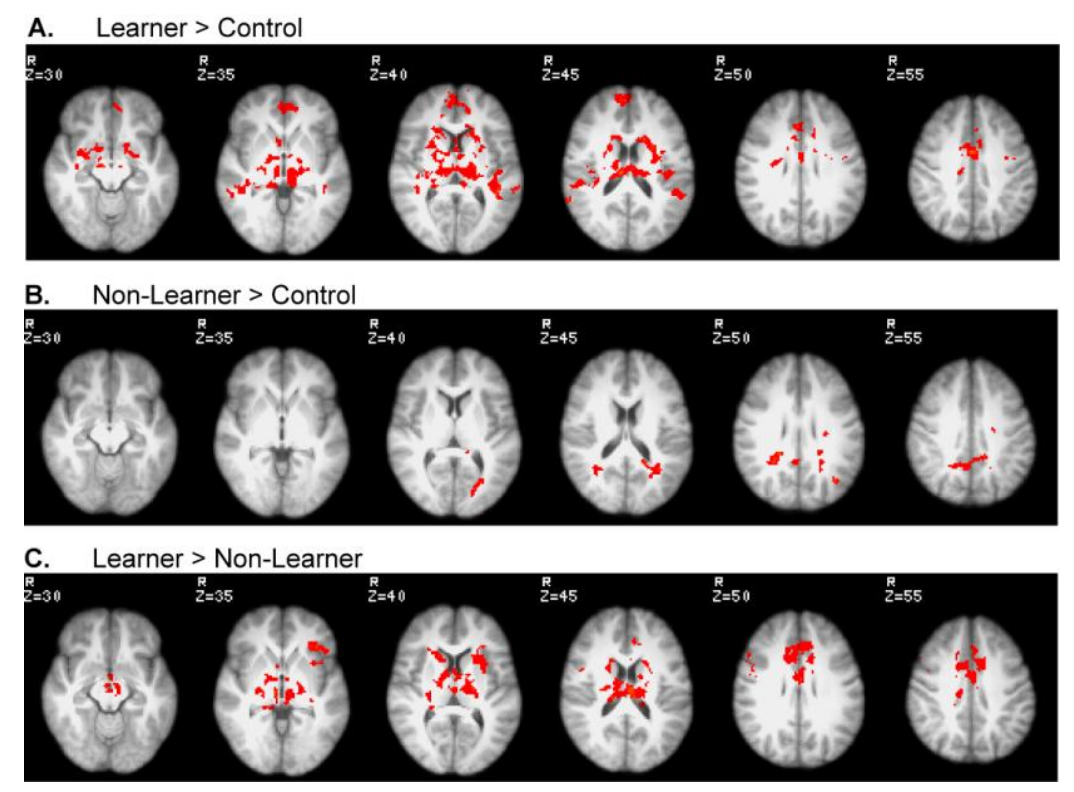

Figure 16: Brain regions significantly activated during the regulation blocks "THINK" vs. "COUNT" after NF training, in A) Learners (post > pre) compared to Controls (post > pre), B) Non-Learners (post > pre) compared to Controls (post > pre), C) Learners (post > pre) compared to NonLearners (post $>$ pre). Images are thresholded at $z>2.3$, a cluster significance threshold of $p=0.05$ (FWE corrected) 
Table 1: Cluster maxima for self-regulation in NF training of the aMCC. Anatomical areas are defined by Harvard-Oxford Cortical and Subcortical Maximum Probability Atlas.

\begin{tabular}{|c|c|c|c|c|c|}
\hline & \multirow{2}{*}{$\begin{array}{l}\text { Number of } \\
\text { voxels }\end{array}$} & \multicolumn{3}{|c|}{ MNI coordinates } & \multirow[t]{2}{*}{ Maximum z } \\
\hline & & $\mathrm{X}$ & $\mathrm{Y}$ & Z & \\
\hline \multicolumn{6}{|l|}{ A. Learner > Control } \\
\hline Frontal pole $\mathrm{R}^{*}$ & 142 & 8 & 56 & 12 & 6.07 \\
\hline Frontal pole L ${ }^{*}$ & 101 & -8 & 66 & 14 & 5.31 \\
\hline Paracingulate gyrus $\mathrm{R}$ & 230 & 8 & 54 & 14 & 5.45 \\
\hline Paracingulate gyrus L & 312 & -4 & 48 & -8 & 4.71 \\
\hline Cingulate gyrus, anterior $\mathrm{R}$ & 294 & 2 & 2 & 38 & 5.12 \\
\hline Cingulate gyrus, anterior $\mathrm{L}$ & 260 & 0 & 0 & 42 & 4.95 \\
\hline Supplementary Motor Area R & 155 & 8 & -2 & 48 & 3.64 \\
\hline Thalamus R & 476 & 8 & -20 & 18 & 4.98 \\
\hline Thalamus L & 616 & -12 & -22 & 2 & 5.03 \\
\hline Caudate R & 141 & 6 & 12 & 2 & 5.26 \\
\hline Caudate L & 62 & -18 & 18 & 4 & 3.20 \\
\hline Putamen R & 176 & 24 & 8 & 6 & 3.64 \\
\hline Putamen L & 73 & -24 & 14 & 6 & 3.44 \\
\hline Pallidum R & 50 & 16 & -4 & -2 & 3.45 \\
\hline Pallidum L & 51 & -16 & -8 & -4 & 3.60 \\
\hline Heschl's gyrus R & 53 & 42 & -22 & 12 & 3.78 \\
\hline Heschl's gyrus L & 95 & -42 & -22 & 12 & 4.80 \\
\hline Planum temporal $\mathrm{R}$ & 137 & 46 & -32 & 16 & 4.54 \\
\hline Planum temporale $\mathrm{L}$ & 180 & -44 & -40 & 10 & 5.35 \\
\hline Opercular Cortex, Parietal R & 41 & 42 & -20 & 16 & 3.83 \\
\hline Opercular Cortex, Parietal L & 25 & -42 & -26 & 16 & 3.60 \\
\hline Opercular Cortex, Central R & 24 & 44 & -14 & 20 & 3.84 \\
\hline Opercular Cortex, Central L & 63 & -42 & -22 & 14 & 4.16 \\
\hline \multicolumn{6}{|l|}{ B. Non-Learner > Control } \\
\hline Precuneus Cortex R & 389 & 10 & -60 & 52 & 4.52 \\
\hline Precuneus Cortex L & 158 & -22 & 54 & 16 & 3.68 \\
\hline Cingulate gyrus, posterior $\mathrm{R}$ & 55 & 18 & -52 & 36 & 3.29 \\
\hline Cingulate gyrus, posterior $\mathrm{L}$ & 78 & -4 & -46 & 34 & 3.59 \\
\hline Lateral Occipital Cortex, superior R & 15 & 12 & -60 & 56 & 3.13 \\
\hline Lateral Occipital Cortex, superior L & 47 & -38 & -68 & 28 & 4.05 \\
\hline Intracalcarine Cortex L & 81 & -28 & -70 & 8 & 3.44 \\
\hline Supracalcarine Cortex R & 11 & 28 & -48 & 22 & 3.62 \\
\hline Supracalcarine Cortex L & 18 & -24 & -62 & 20 & 3.00 \\
\hline Angular gyrus $\mathrm{L}^{\dagger}$ & 35 & -34 & -54 & 18 & 3.41 \\
\hline Angular gyrus $\mathrm{R}^{\dagger}$ & 17 & 28 & -52 & 32 & 3.53 \\
\hline \multicolumn{6}{|l|}{ C. Learner > Non-Learner } \\
\hline Cingulate gyrus, anterior $\mathrm{R}$ & 495 & 12 & 6 & 36 & 4.53 \\
\hline Cingulate gyrus, anterior $\mathrm{L}$ & 525 & -2 & -2 & 40 & 4.46 \\
\hline Paracingulate gyrus $\mathrm{R}$ & 273 & 4 & 30 & 32 & 5.42 \\
\hline Paracingulate gyrus L & 342 & -10 & 34 & 32 & 3.97 \\
\hline Thalamus $\mathrm{R}$ & 289 & 14 & -6 & 6 & 5.18 \\
\hline Thalamus L & 494 & -8 & -4 & 0 & 4.78 \\
\hline $\begin{array}{l}\text { Inferior Frontal Gyrus pars } \\
\text { triangularis L }\end{array}$ & 15 & -20 & 30 & 4 & 5.28 \\
\hline Insula Cortex L & 227 & -28 & 14 & 14 & 4.86 \\
\hline Frontal Operculum Cortex L & 63 & -32 & 24 & 12 & 3.62 \\
\hline
\end{tabular}




$\begin{array}{llllll}\text { Frontal Orbital Cortex L } & 153 & -32 & 30 & 4 & 4.61 \\ \text { Precentral gyrus R } & 456 & 42 & -12 & 64 & 4.56 \\ \text { Supplementary Motor Area R } & 251 & 12 & 2 & 54 & 4.55 \\ \text { Supplementary Motor Area L } & 115 & -14 & 2 & 42 & 3.21 \\ \text { Superior Frontal Gyrus R } & 208 & 12 & 14 & 64 & 4.31 \\ \text { Superior Frontal Gyrus L } & 123 & -16 & 8 & 66 & 3.95 \\ \text { Middle Frontal Gyrus R } & 137 & 54 & 20 & 30 & 4.08 \\ \text { Pallidum R } & 52 & 22 & -4 & 2 & 3.64 \\ \text { Pallidum L } & 27 & -20 & -14 & -2 & 3.32 \\ \text { Caudate R } & 167 & 14 & 18 & 12 & 4.12 \\ \text { Caudate L } & 22 & -14 & 4 & 22 & 3.20 \\ \text { Putamen L } & 19 & -24 & 14 & 6 & 3.40 \\ \text { Cingulate gyrus, posterior R } & 113 & 12 & -20 & 40 & 3.62\end{array}$

* part of the ventromedial prefrontal cortex

+ part of the inferior parietal lobule

\subsubsection{Feedback appraisal: group anallysis}

Whole-brain analysis on changes in feedback appraisal activity within the ST group in the last compared to the first training run showed no significant results, possibly indicating similar pattern of brain activity during the two sessions. Subsequent analysis focused on the two runs separately. Brain regions that are active during feedback appraisal are shown in Figure 17. Both the last and the first training run showed similar pattern of brain activation in the pregenual ACC (pgACC), precuneus, PCC, and IPL brain areas belonging to the Default Mode Network (DMN). The coordinates, size and significance level ( $z$-score) of activation clusters are available in Table 2. 
A. Training 1

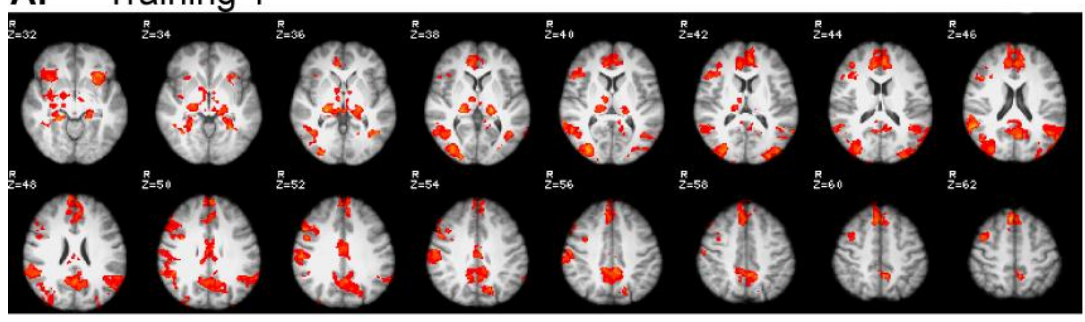

C. $\mathrm{DMN}$

B. Training 18
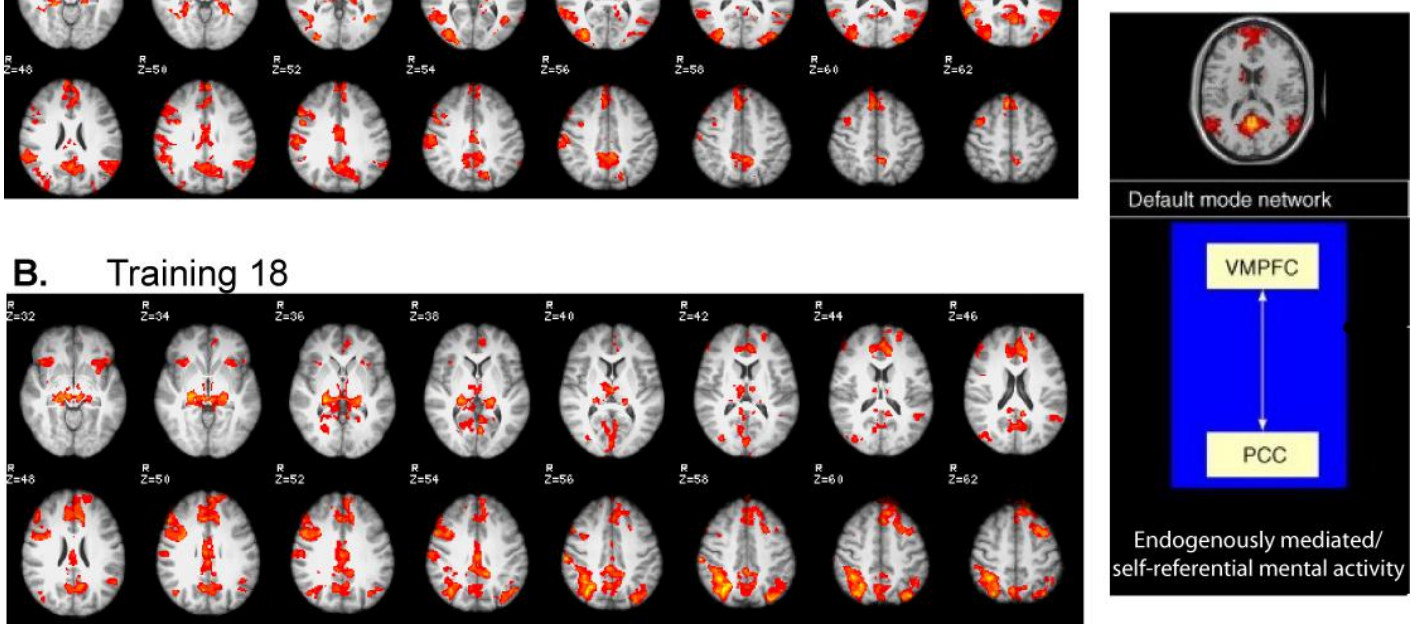

Figure 17: Brain regions significantly activated during the feedback appraisal blocks "FEEDBACK" vs. "COUNT" during A) the first training run, and B) the last training run. Images are thresholded at $z>2.3$, a cluster significance threshold of $p=0.05$ (FWE corrected). C) Brain regions belonging to the DMN (adapted from Bressler \& Menon, 2010). Reprinted from Trends in Cognitive Sciences 14(6), copyright 2010, with permission from Elsevier. 
Table 2: Cluster maxima for feedback-appraisal in NF training of the aMCC. Anatomical areas are defined by Harvard-Oxford Cortical and Subcortical Maximum Probability Atlas.

\begin{tabular}{|c|c|c|c|c|c|}
\hline & \multirow{2}{*}{$\begin{array}{l}\text { Number of } \\
\text { voxels }\end{array}$} & \multicolumn{3}{|c|}{ MNI coordinates } & \multirow[t]{2}{*}{ Maximum z } \\
\hline & & $x$ & $Y$ & Z & \\
\hline \multicolumn{6}{|l|}{ A. Training 1 (first training) } \\
\hline Precuneus Cortex R & 555 & 2 & -60 & 34 & 3.54 \\
\hline Precunues Cortex L & 1152 & -6 & -46 & 42 & 4.04 \\
\hline Cingulate gyrus, posterior $\mathrm{R}$ & 310 & 10 & -40 & 40 & 3.59 \\
\hline Cingulate gyrus, posterior $\mathrm{L}$ & 493 & -4 & -44 & 40 & 3.75 \\
\hline Cingulate gyrus, anterior $\mathrm{R}$ & 475 & 8 & 36 & 16 & 3.80 \\
\hline Cingulate gyrus, anterior $\mathrm{L}$ & 350 & -4 & 42 & 16 & 3.66 \\
\hline Paracingulate gyrus, $\mathrm{R}$ & 451 & 4 & 30 & 44 & 3.51 \\
\hline Paracingulate gyrus, L & 467 & -6 & 46 & 14 & 3.94 \\
\hline Superior frontal gyrus, $\mathrm{R}$ & 640 & 6 & 32 & 50 & 4.18 \\
\hline Superior frontal gyrus, L & 383 & -2 & 56 & 26 & 3.75 \\
\hline Frontal Pole R & 438 & 2 & 60 & 20 & 3.70 \\
\hline Frontal Pole L & 192 & -2 & 58 & 28 & 3.82 \\
\hline Lateral occipital cortex, superior $\mathrm{R}$ & 647 & 30 & -84 & 18 & 4.00 \\
\hline Lateral occipital cortex, superior L & 460 & -42 & -86 & 12 & 3.65 \\
\hline Lateral occipital cortex, inferior R & 465 & 32 & -84 & 6 & 4.50 \\
\hline Lateral occipital cortex, inferior L & 210 & -44 & -62 & 2 & 3.78 \\
\hline Angular gyrus $\mathrm{R}$ & 350 & 56 & -50 & 20 & 4.29 \\
\hline Angular gyrus L & 550 & -46 & -60 & 20 & 3.82 \\
\hline Supramarginal gyrus, posterior $\mathrm{R}$ & 164 & 52 & -44 & 22 & 4.17 \\
\hline Supramarginal gyrus, posterior L & 95 & -40 & -50 & 16 & 3.64 \\
\hline Thalamus $\mathrm{R}$ & 434 & 22 & -28 & 2 & 4.22 \\
\hline Thalamus L & 304 & -18 & -28 & 4 & 4.17 \\
\hline Middle Frontal gyrus R & 614 & 42 & 8 & 54 & 4.21 \\
\hline Insula Cortex L & 192 & -32 & 14 & -16 & 4.09 \\
\hline Insula Cortex R & 180 & 34 & 18 & -10 & 3.74 \\
\hline \multicolumn{6}{|l|}{ B. Training 18 (last training) } \\
\hline Precuneus Cortex R & 833 & 6 & -66 & 28 & 3.67 \\
\hline Precuneus Cortex L & 1048 & -10 & -60 & 24 & 3.82 \\
\hline Cingulate gyrus, posterior $\mathrm{R}$ & 646 & 2 & -20 & 30 & 4.32 \\
\hline Cingulate gyrus, posterior $\mathrm{L}$ & 682 & -12 & -44 & 36 & 3.98 \\
\hline Cingulate gyrus, anterior $\mathrm{R}$ & 390 & 6 & 30 & 28 & 4.30 \\
\hline Cingulate gyrus, anterior $\mathrm{L}$ & 429 & -10 & 34 & 18 & 3.83 \\
\hline Paracingulate gyrus, $\mathrm{R}$ & 405 & 4 & 30 & 30 & 4.15 \\
\hline Paracingulate gyrus, L & 806 & -12 & 30 & 24 & 3.80 \\
\hline Superior parietal lobule R & 851 & 36 & -50 & 52 & 4.90 \\
\hline Frontal Pole L & 612 & -14 & 44 & 52 & 3.89 \\
\hline Lateral occipital cortex, superior $\mathrm{R}$ & 1730 & 24 & -58 & 46 & 4.85 \\
\hline Lateral occipital cortex, superior L & 941 & -34 & -76 & 44 & 4.14 \\
\hline Angular gyrus $\mathrm{R}$ & 289 & 38 & -52 & 42 & 4.35 \\
\hline Angular gyrus L & 328 & -56 & -54 & 24 & 3.22 \\
\hline Supramarginal gyrus, anterior $\mathrm{R}$ & 255 & 62 & -22 & 40 & 4.69 \\
\hline Supramarginal gyrus, posterior $\mathrm{R}$ & 319 & 38 & -46 & 42 & 4.28 \\
\hline Thalamus $\mathrm{R}$ & 499 & 20 & -26 & -6 & 4.38 \\
\hline Thalamus L & 408 & -18 & -32 & 4 & 4.06 \\
\hline Middle Frontal Gyrus R & 620 & 54 & 30 & 28 & 4.10 \\
\hline Middle Frontal Gyrus L & 555 & -36 & 10 & 58 & 4.11 \\
\hline Insula Cortex L & 168 & -28 & 14 & -14 & 3.37 \\
\hline Insula Cortex R & 193 & 28 & 12 & -12 & 3.24 \\
\hline
\end{tabular}




\subsubsection{Self-reports: group analysis}

Data presented in this section are from the questionnaires given to the subjects after each pre-training, NF training and post-training sessions. The median values of the ratings of the difficulty and performance of the up-regulation "Think", and downregulation "Count" phases in the ST and PT group are illustrated in Figure 18. Wilcoxon Signed Ranks Test test comparing the post- to the pre-training session revealed that, for the ST group, difficulty of the up-regulation phase was rated as significantly less "difficult" (Mdn = 'Neither', p = 0.008) (Figure $18 \mathrm{~A}$ ), performance of up-regulation phase was rated as significantly more successful (Mdn = 'Successful', p =0.034) (Figure $18 \mathrm{~B})$, but the difficulty of the down-regulation phase was rated as more difficult (Mdn = 'Neither', $p=0.02$ ) (Figure $18 \mathrm{C}$ ).

On the other hand, there were no significant differences in the how the PT group ranked the difficulty of "Think" phase, difficulty of "Count" phase, performance of "Think" phase and performance of "Count" phase in the post-training session compared to the pre-training session. Analyses based on learning status in the post-training session compared to the pre-training session, revealed that for the Non-Learners, the only significant difference in rating was the difficulty of the up-regulation phase, which was rated as significantly less difficult. For the Learners, there was no significant difference in ratings of the four parameters. 


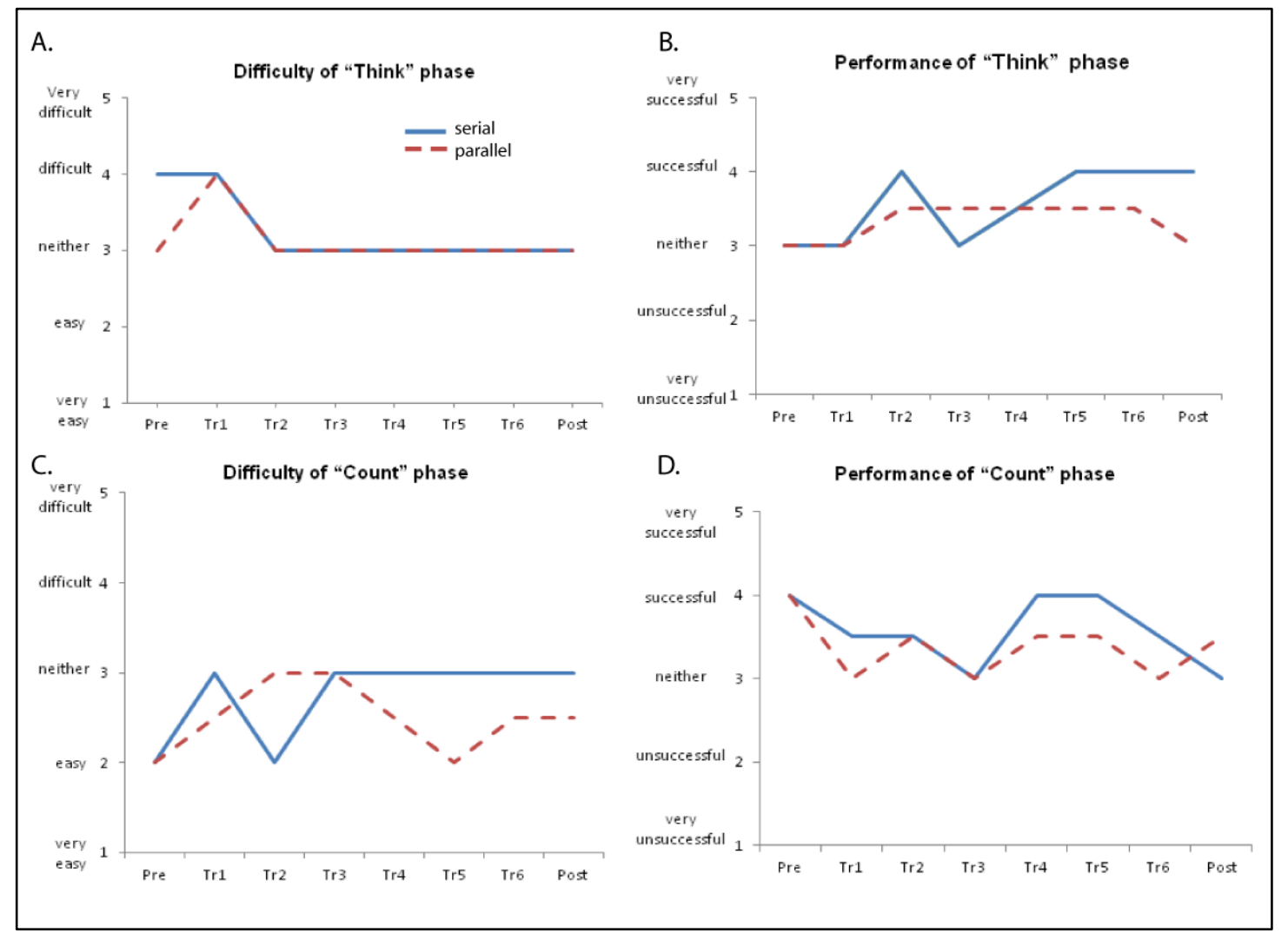

Figure 18: Median values of self-reported difficulty and performance rating in ST and PT group.

Analysis on the ratings of valence and arousal in the post-training session compared to the pre-training session revealed no significant differences in the ratings by the PT group, the ST group, the Learners and the Non-Learners. Interestingly, for the ratings of dominance, the ST group and the Learners rated feeling more dominant in the post-training session ( $\mathrm{Mdn}=3.5, \mathrm{p}=0.014$ and $\mathrm{Mdn}=4.0, \mathrm{p}=0.025$ respectively).

\subsubsection{Use of mental strategies}

The mental strategies used by the subjects in the up-regulation and down-regulation phase were also documented. The mental strategies reported by subjects to up-regulate their aMCC activity range from cognitive strategies: " planning daily activities", "spelling long words", "thinking of vocabulary in a foreign language"; positive emotions imagery: "positive thoughts about my partner, my family"; negative emotions imagery: "arguments with former partner", visual mental imagery: "playing Tetris games", "playing driving computer games". Most of the Learners (6 out of 8 ) had used cognitive imagery. However, there are no differences in the type strategies used by the Learners and NonLearners in the up-regulation phase of NF training. For the down-regulation phase, most 
subjects reported having performed backward counting while trying to relax. A few subjects had used breath control techniques to down-regulate their aMCC activity.

Five out of eight Learners had some exposure to meditation training, ranging from one-off ("..took autogenic training course for 3 months") to regular ("..been doing Yoga since four years"). The Learners who had meditation training were the ones who used breath control techniques to down-regulate their aMCC activity in the "Count" phase. 


\subsection{Discussion}

To the best of the author's knowledge, this is the first extensive NF study of the cingulate cortex, which used 18 runs of NF training over the span of two weeks, compared two NF paradigms, included untrained controls, examined the transfer condition, included questionnaires and examined two behavioral tests, all in one study. Specifically, this study explored rt-fMRI NF of the aMCC in healthy subjects by assigning two different paradigms: the conventional Parallel paradigm, in which strategy search and feedback is temporally coupled, compared to a novel Serial paradigm in which strategy search and feedback are temporally uncoupled. This study found that the Serial paradigm is promising in facilitating NF of the aMCC.

The ability to self-regulate the aMCC in the presence and absence of feedback is higher in the ST than in the PT group. Most Learners originate from the ST group. Learners indeed showed successful retention of the ability to self-regulate aMCC activity in the absence of feedback and had more ETRs during the NF training than NonLearners. There is a correlation between transfer success and ETR. Compared to the Controls or Non-Learners, Learners activated brain regions related to reward learning in addition of the aMCC itself. There is no apparent difference in the use of mental strategies between the Learners and Non-Learners. The ST group and the Learners reported feeling more in control of the assigned NF paradigm than the PT group and the Non-Learners. It appears that feedback appraisal elicited lower aMCC activity than selfregulation and there was no apparent difference in aMCC activity in Learners and NonLearners. Feedback appraisal activated brain regions that are nodes of the DMN.

\subsubsection{Neurofeedback learning}

\subsubsection{Training and transfer}

One of the goals in a NF study is to demonstrate NF-induced learning. The definition of NF-induced learning itself is diverse in the NF literature, and has been attempted on multiple levels. In NF studies of the cingulate cortex, at the very basic level, learning has been defined as successful regulation of the target ROI in the regulation blocks compared to the control blocks in one NF session (Mathiak et al., 2010; Gröne et al., 2014). On the next level, learning can be seen as increased control of the target ROI over multiple training runs (Weiskopf et al., 2003; deCharms et al., 2005). Another index of learning is based on the transfer condition, i.e. the ability to retain self-regulation in the absence of feedback (Mathiak et al., 2010; Hamilton et al., 2011). Out of these two 
studies, only one NF study (which used just one subject) showed success in selfregulation in the absence of feedback. (Mathiak et al., 2010). On the next level in this category, learning index can be defined based on comparison to an untrained control group, which is not expected to show NF-induced learning. It is this criteria that was have used in this study to define NF-induced learning. It is difficult to draw a parallel to other NF studies of the cingulate cortex because those studies have used either small number of subjects, included no transfer run, used relatively fewer training runs, or excluded controls. Due to these differences, naturally the yardstick to define NF success differs between studies. Nevertheless, the yardstick used in this study is the most conservative in comparison to other studies.

In the current study, group analysis of the transfer condition comparing trained and controls revealed that there was no NF-induced learning in the trained group. Group analysis of the training condition also did not show significant increase over time in both ST and PT group. These results were due to the high variability of the BOLD aMCC activity within the group, attributed to the presence of Non-Learners amongst Learners in one paradigm.

These results indicate that in general, self-regulation of the aMCC is challenging. Unlike the sensorimotor cortex where motor imagery (e.g. imagine playing the piano) can be used as a strategy there are no clear-cut strategies that can be prescribed that voluntarily activate the ACC (Mathiak et al., 2010). Nevertheless, in this study, there is an indication that the Serial paradigm could help facilitate performing NF, as it elicited significantly higher BOLD aMCC changes in the ST group compared to the PT group in the transfer and the training condition, which could be driven by a greater presence of Learners in the ST group (6 out of 10) than in the PT group (2 out of 10).

\subsection{Controls}

There are various types of controls that can be used in a NF study, depending on the objective of the study. With the exception of one study, (deCharms et al., 2005), most NF studies of the cingulate cortex did not even include a control group. In this exploratory study, an untrained control group was used to examine whether any learning effects observed in the trained group are due to undergoing a 2-week NF training or have to be ascribed to merely performing the same task twice. Both SC and PC groups in this study did not show significant changes from the pre- to the post- measurements in the transfer condition, consistent of what is expected of such a control group. Therefore, these 
controls were then used to establish a baseline against which performance of subjects in the trained group during transfer and training could be compared directly. A limitation with the controls is that, only 5 controls were included in each paradigm (due to limitations in measurement time), half the sample size of the experimental group.

\subsubsection{Performance-based categorization of subjects}

Henceforth, to gain more insights into the neural correlates associated with successful NF learning, subjects were re-grouped based on their performance, into Learners and Non-Learners, independent of paradigm. The criteria to define Learners was based on individual comparison to the untrained control group which is not expected to show NF training-related changes in BOLD aMCC activity. A simple threshold comparison was chosen instead of a statistically significant comparison because the latter was too conservative for these exploratory data. Using this criterion, it has been revealed that more Learners came from the ST group than the PT group, although statistical tests failed to show significant association between paradigm and learning status, which is most likely attributed to the small sample size in each category.

Compared to the Non-Learners, the Learners were successful in using the feedback to self-regulate their aMCC activity, evident by the significant higher change in BOLD aMCC activity over NF training runs compared to the Non-Learners. Learners were also successful in retaining the skill to self-regulate the aMCC in the absence of the feedback. These results were expected since the Learners were defined based on the criteria of showing increased BOLD aMCC activity more than that of the control group in the transfer condition.

\subsection{Efficient training runs and successful transfer}

A possible predictor for the outcome of this NF study of aMCC self-regulation is the number of ETR, in which the data showed a significant difference between Learners and Non-Learners. Learners showed double the number of ETR than Non-Learners, and there is a moderate correlation between ETR and transfer success. Although it may be tempting to associate higher number of ETR with higher PSC difference, in reality, transfer success in each subject is variable. Some subjects acknowledged being distracted by the feedback, and performed better without it, i.e. showing transfer success with only some ETR. Conversely, some subjects seemed efficient in using the feedback to self-regulate the aMCC but were not successful once the feedback was 
absent e.g. having 17 ETR but showed no transfer success. Nevertheless, the present results suggest that Learners in general are able to use NF more efficiently to help their attempt in self-regulation and could subsequently adapt that acquired ability to a slightly different situation (in this case, performing self-regulation in the absence of feedback).

\subsubsection{Learning curves of NF training}

In the NF literature, characteristics of a NF learning curve have been described as positive exponential function (Birbaumer et al., 2013), inverted U-shape (Lee et al., 2011), and a linear increase (Lawrence et al., 2014; Young et al., 2014). In general, it is agreed that learning involves incremental changes over time.

Group analysis of the training condition revealed that neither the ST nor the PT group showed a linear increase in the aMCC BOLD activity over time. Analysis on individuals showed that there are some subjects who demonstrated a clear increase in aMCC activity over time (e.g. individual L1 and L4 in Figure 12). However, not all learners showed a linear increase of aMCC activity over 18 NF training runs. Therefore, to describe learning in NF of the aMCC, ETR may be a better index than presence of a linear learning curve.

\subsubsection{Brain areas and networks involved in NF learning}

To explore the neural correlates of NF learning, whole-brain fMRI analysis was performed to investigate activations beyond the target ROI. The results show that successful self-regulation of the aMCC brought about changes in brain regions implicated in reward learning. In addition to increasing BOLD activation of the aMCC, compared to the Controls or Non-Learners, the Learners had more activity in the basal ganglia - a brain region related to learning and motivation; and in the thalamus - an essential relay station of the cortico-basal ganglia circuit, integrating cortical inputs to drive goal-directed behavior.

This finding supports the view that the basal ganglia play an important role in NF-based learning which is often viewed as operant learning (Koralek et al., 2012; Birbaumer et al., 2013). Specifically, the caudate nucleus and putamen, sub-regions of the dorsal striatum of the basal ganglia, have a role in goal-directed operant learning (Delgado et al., 2005; Liljeholm and O'Doherty, 2012). A recent study of NF training of the nucleus accumbens (part of the ventral basal ganglia) using short phases of self- 
regulation (10 s), showed activation in caudate and putamen during NF training in addition to the target ROI (Greer et al., 2014).

Increased activation of the thalamus in the Learners is also in line with the role of the thalamus as an essential hub of integration of networks that underlie the ability to regulate behavior (Haber and Calzavara, 2009). In operant learning (also called instrumental learning), individual's behavior is modified by its consequences. In terms of the present study, the Learners have learnt the skill to self-regulate their brain activity by associating the reward (feedback from the aMCC) with their behavior (strategies). Even when no feedback is presented in the transfer task, the acquired selfregulation skill is maintained and can be applied. To do so, Learners must be flexible in finding an appropriate response in an unfamiliar situation or breaking a habitual response (e.g. accustomed to regulating with feedback during the NF training) when contingencies change in familiar situation (e.g. regulating without the usual feedback in the post-training session) (Gazzaniga et al., 2009). This requires continuous processing of information from limbic, cognitive and motor circuits; and subsequent functional integration of that information, which is mediated by the thalamus.

It should also be mentioned that the post-training transfer session was not even done immediately after the last training, but on average after three days, therefore suggesting that NF-learning effects can persist at least for a few days.

\subsubsection{Serial versus Parallel paradigm}

The Serial paradigm was developed to overcome the challenges of performing rt-fMRI NF of the aMCC as layed out in the introduction. The idea of presenting the feedback only after the regulation phase instead of concurrently, was based on the challenges of rtfMRI NF and the aim of training a brain area involved in cognition. Some insight came from the paradigm used in a study on direct instrumental conditioning of the sensorimotor cortex (Bray et al., 2007). Later during the course of the measurements, a study that addressed similar issues to the present study emerged. Johnson et al. (2012) did a pilot study of a similar "intermittent" vs. "continuous" rt-fMRI NFB study of the premotor cortex and showed that intermittent, temporally separated presentation of feedback (about $20 \mathrm{~s}$ delay) is more effective than continuous presentation in a motor imagery task (Johnson et al., 2012).

The intermittent feedback presentation reduces the processing of the cognitive load from parallel to serial. In serial processing, the self-regulation phase is uncoupled 
from the feedback appraisal phase. Therefore subjects can process these two phases separately, and consequently, are less burdened in doing a NF task. A serial paradigm as such reduces the interference between self-regulation and feedback appraisal.

\subsubsection{Temporal contiguity of NF paradigms}

Although the feedback in the Serial paradigm is considerably delayed and nonconcurrent with the self-regulation phase, six out of ten subjects were able to learn how to control the delayed BOLD feedback signal of their aMCC activity. The findings in this study on delayed feedback are in support of the NF study of motor imagery using $\sim 20 \mathrm{~s}$ of delayed feedback (Johnson et al., 2012), and a connectivity-based NF using 60 s delay (Koush et al., 2013). In both studies, subjects managed to learn how to control their brain activity represented by the delayed feedback signal that was not presented in parallel with the task of self-regulation.

The issue of temporal contiguity in NF has just emerged in the recent years, thus NF studies addressing the issue are scarce. However, insights on temporal contiguity and learning can be drawn from the experimental psychology literature on causal learning. Studies on delayed reinforcement have shown that the structure of a trial has to be apparent to the subjects in order to facilitate learning (Greville et al., 2013). In addition, temporal predictability - the consistency or regularity of the time interval separating events, has been shown to facilitate causal learning (Greville \& Buehner, 2010). In the context of the current study, one explanation on why the Serial paradigm can facilitate NF learning, is that the feedback has an apparent onset and duration, and its temporal predictability is higher than that of the Parallel paradigm.

It is not exactly known how the Learners in the PT group approach the NF task. The conventional way of doing it is by self-regulating in parallel of appraising the feedback. However, another possible strategy to deal with the Parallel paradigm is that subjects could use a workaround strategy to lessen the interference between those two phases by: self-regulating in the first half of each regulation blocks, and appraising the feedback in the second half - similar to the uncoupling of the two phases in the Serial paradigm. More research would be necessary to investigate this interesting question.

\subsubsection{Cognitive load of NF paradigms}

Feedback presented in parallel to executing the strategy can be distracting, and one author has explicitly raised a concern about this (Robineau et al., 2014). In the Serial 
paradigm, subjects could be more focused in applying their mental strategy without being distracted by the feedback unlike in the Parallel paradigm. In essence, subjects in the PT group viewed a more complex feedback because they need to associate the feedback with strategies that they have applied $\sim 6-8$ seconds earlier, while simultaneously self-regulating the activity of the aMCC. If such temporal contiguity (as discussed in the previous section) can be tolerated, the cognitive load is less so. One EEG study has shown that increased cognitive load of the feedback signal, (through making the feedback not directly interpretable), impairs learning efficiency in the medial-frontal system due to reduced ability to process feedback (Krigolson et al., 2012).

Self-reports about difficulty and performance give a clue to the perception of cognitive load of the NF paradigms. Significant changes in ratings towards the paradigm being less difficult and the performance being more successful in the post-session were only observed in the ST group and not the PT group, suggesting a more uniform view on the demands of the Serial paradigm in each session. Some subjects in the PT group also verbally remarked on the difficulty of parallel feedback although there was no a specific question on this aspect in the administered self-report questionnaire.

\subsubsection{Feedback appraisal}

As a consequence of the design of the Serial paradigm, the feedback appraisal can be investigated separately, in addition to the self-regulation phase. Feedback appraisal elicited significantly lower BOLD activity within the aMCC compared to that of selfregulation.

The potential conflict between BOLD activity attributed to self-regulation of the aMCC, and attributed to feedback appraisal by the aMCC, was one of the fundamental factors why the Serial paradigm was conceived. Results of this study may suggest that activity of aMCC that is being trained is functionally specific, i.e. training self-regulation activity of the aMCC does not appear to result in a concomitant increase of feedback appraisal activity in the aMCC. Furthermore, comparable aMCC activity in Learners and Non-Learners during feedback appraisal suggests that difference in performance might not appear to be attributed to the activity of appraising feedback. This study also showed that feedback appraisal-related BOLD activity in the aMCC is comparable to that reported by Amiez and colleagues, which is in the range of $0.1-0.2 \%$ (Amiez et al., 2013).

Whole-brain fMRI analysis sheds more light onto the neural correlates of feedback appraisal beyond the aMCC. There is no difference in brain activity elicited by 
feedback appraisal in the last training run compared to the first training run, due to similar pattern of brain activations in the two runs. The brain areas activated during feedback appraisal resemble the nodes of the DMN. DMN is a set of regions which are more active at rest than during goal-directed task performance (Fox and Raichle, 2007). Moreover, results of this present study are in concordance with the function of the DMN in internal mentation, i.e. spontaneous introspective thoughts (Andrews-Hanna, 2012). When viewing the feedback, subjects naturally would engage in self-referential, introspective thoughts, i.e. wondering about their performance during the preceding self-regulation phase and planning how to improve it in the next self-regulation phase. Indeed this is what has been reported by some subjects in the post-session questionnaire.

\subsubsection{Self-reports}

In addition to the objective measures of performance, self-report questionnaires can provide valuable insights into the subjective experience, from the perspective of the participants. This study did not use a cross-over design, therefore one subject only experienced one paradigm, so only within-group comparison of the ratings of difficulty and performance across two-time points (pre-training and post-training) could be done. A suitable non-parametric statistical test to analyze repeated measures, between-group comparison for ordinal data was not available.

The self-report questionnaires provide a hint that supports the hypothesis that the Serial paradigm facilitates learning self-regulation. Ratings of dominance by the ST group and in the Learners increased after undergoing the 2-week NF training, indicating that they felt more in control of the NF task. For future experiments, more questions may be incorporated, for example, asking the subjects if they managed to stay focused, at which session approximately did they think they were able to gain control of their aMCC activity, and how helpful was the feedback, e.g. rating it from "distracting" to "helpful".

\subsubsection{Mental strategies}

Another interesting aspect of the self-report is the open-ended question on mental strategies. There was no clear difference in the mental strategies reported by Learners and Non-Learners. Similarly, a rt-fMRI NF study on visual cortex has shown that, learners and non-learners did not differ in their reported strategies or their attentional effort (Scharnowski et al., 2012). It could be the case that the questionnaires used in the present study were not sensitive enough to distinguish subtle differences between the 
groups. Another reason is that it may be that the Learners just could not verbalize specific mental strategies because the process of self-regulation might have become automated, as observed in an EEG-NF study on spontaneous mental strategies (Kober et al., 2013).

Although strategies were suggested, subjects were free to develop their own strategies in their attempt to self-regulate the aMCC. For example, a few Learners had used breath control techniques to lower the aMCC activity during the "COUNT" phase, instead of using the author's suggestion to count backwards. One NF study has shown that explicit instructions on how to control one's brain activity could even be counterproductive to achieving the "focused but relaxed" mental state required for selfregulation learning in NF (Kober et al., 2013). Such mental state appears similar to what is trained in meditation. In fact, neurofeedback and meditation have been viewed as methods to train mental states (Brandmeyer and Delorme, 2013). Furthermore, shortterm meditation training has been shown to improve self-regulation in cognition and emotion (Tang et al., 2007). In this present study, half of the learners have had some exposure to gaining attentional control or self-awareness through meditation or yoga. It may be that those who had such exposure find it easier to achieve the mental state required in self-regulation, hence performed better than the others. However, since this study did not systematically investigate the experience level, type, or duration of their meditation training (as these were beyond the scope of this current study), this notion remains to be investigated further.

\subsection{Conclusions}

Learning to self-regulate aMCC activity through rt-fMRI NF is in general, difficult yet possible. This study has shown that the Serial paradigm is promising in facilitating learning in rt-fMRI neurofeedback through reducing the cognitive load of the NF task, and having a clear temporal contiguity. The author also advocates the need to analyze and present individual data for a more comprehensive understanding about individual differences, and the factors driving NF success. This study has provided evidence that learning to self-regulate the aMCC not only brought about changes in the activity of the aMCC, but also engages other brain regions involved in reward learning. Learning selfregulation most likely does not depend on the content of the mental strategies per se, but rather the ability to achieve of a focused and attentive mental state. In addition, this 
study demonstrated that feedback appraisal can be characterized as a self-referential activity due to the engagement of the DMN. 
Generalization of NF 


\section{Generalization of neurofeedback training to behavior}

\subsection{Introduction}

\subsubsection{Effects of neurofeedback learning on behavior}

In a NF study, following training of self-regulation, one can test if the learned regulation brings about specific behavioral effects. This phenomenon, also called "generalization", can be investigated through behavioral tests administered before and after learning. Testing the behavioral effects of learned self-regulation is part of the experimental framework of NF (Sulzer et al., 2013). In the rt-fMRI NF literature, behavioral test can refer to either specific cognitive paradigms involving a behavioral response or self-reports. The self-reports used in this study (Section 3.2.8) intended to probe the subject's perception to the paradigm and difficulty of task; and therefore were not a direct measure to test the effects of NF learning. This chapter discusses behavioral paradigms as a test for the effects of NF learning.

Behavioral tests involving specific cognitive paradigms have been performed while undergoing fMRI measurement (Mathiak et al., 2010), or in the scanner without concurrent fMRI measurement (Chiew et al., 2012; Robineau et al., 2014). Behavioral tests involving self-ratings have been administered either inside (Caria et al., 2010) or outside the MRI scanner (deCharms et al., 2005; Ruiz et al., 2013). In our present study, the subjects performed two cognitive paradigms, CPT and Flanker task, while undergoing fMRI measurement.

The behavioral tests selected usually intend to test the effects in the domain that has been trained in the NF training. The pioneering rt-fMRI NF study of the ACC used the self-assessment manikins (SAM) (Bradley and Lang, 1994) to assess affective states (specifically emotional valence and arousal) after each session and separately after each block (Weiskopf et al., 2003). Similar ratings of valence and arousal have also been used in one NF training of the rostral ACC but as an implicit measure to judge prosody of pseudowords (Gröne et al., 2014). In NF training of the ACC in chronic pain patients, perception of pain has been used as an index of generalization of NF learning (DeCharms et al., 2004). In addition to the present study, there is only one other NF study of the ACC which used a behavioral test 
specifically testing the cognitive control domain (Mathiak et al., 2010). This study used the Simon task - a task of similar nature to the Flanker task.

\subsubsection{CPT}

The CPT is a group of cognitive paradigms for evaluating sustained attention (Riccio et al., 2002). The principle concept of the CPT paradigm involve sustaining attention to a fast stream of continuous stimuli presentation and responding to a specific "target" stimulus presented in a specific pattern. Various properties of anticipation can be indexed by different contrasts; for example, early and late anticipation (Luetcke et al., 2009). Performance in the CPT is sensitive to brain damage or dysfunction affecting the attention system and thus this task has been used heavily in clinical populations. In the present study, besides employing CPT as a behavioral task to test learning effects of NF, the same task has also been used as a functional localizer task for the aMCC (Section 2).

\subsubsection{Flanker task}

The Flanker task is a cognitive paradigm for examining conflict resolution and response inhibition (Nee et al., 2007). In a standard Flanker task (also called Eriksen Flanker task), subject responds as fast as possible to a target letter embedded in a string of letters called "flankers" (Eriksen and Eriksen, 1974). In a congruent condition, the target letter and the string of flankers signify the same response; and conversely in the incongruent condition, they signify an alternative response. In the incongruent condition relative to the congruent condition, it would be expected that the reaction time increases, and accuracy decreases.

\subsubsection{Aims}

This study investigated possible effects of successful self-regulation of the aMCC to the CPT and Flanker task at the level of behavioral measures (reaction time and accuracy) and whole brain (BOLD activity changes). 


\subsection{Materials and methods}

\subsubsection{Participants}

The subjects in this study were the same set of subjects that participated in the NF training study. Please refer to Section 3.2.1 for more details.

\subsubsection{Experimental procedure overview}

The CPT and Flanker behavioral tasks were implemented as part of the NF training study described in Chapter 3 (Section 3.2.2) and were performed in the pretraining and post-training session. In the pre-training session, subjects first underwent one fMRI run performing the CPT and one run performing the Flanker task prior to the pre-training NF run. Before subjects entered the scanner they underwent a short demonstration of all tasks to assure full understanding. In the post-training session, subjects performed one fMRI run of CPT and the Flanker task after the post-training run. In both sessions, CPT was always performed prior to the Flanker task to allow real-time fMRI monitoring with TBV software.

\subsubsection{MRI data acquisition}

All BOLD fMRI measurements were obtained by a gradient-echo EPI sequence (TR = $2 \mathrm{~s}, \mathrm{TE}=36 \mathrm{~ms}$, flip angle $=70^{\circ}$, acquisition matrix $\left.=96 \times 96\right)$ at $2.0 \times 2.0 \times 4.0 \mathrm{~mm}^{3}$ resolution with 22 slices oriented along the AC-PC line, encompassing the cerebrum until the mid-brain. Individual slice positions from the first fMRI scanning session were saved and subsequently re-applied in all sessions to minimize slice positioning differences between datasets. Motion correction on fMRI data was performed in kspace (online software of the manufacturer).

\subsubsection{Behavioral paradigms and task}

For both CPT and Flanker task, visual stimuli were projected onto a screen inside the MRI scanner bore, and viewed by the subjects through a mirror mounted on top of the head coil. All stimuli were presented as white letters on a black background

\section{(Figure 19).}

\subsubsection{CPT paradigm}

The CPT O-X-H paradigm was implemented both as a functional localizer, and as a behavioral test for the effects of NF learning. The details of the CPT paradigm are 
described in Section 2.2.4. Online rt-fMRI monitoring of the BOLD activity was performed using TBV.

\subsubsection{Flanker paradigm}

The Flanker paradigm was presented using MATLAB Psychophysics Toolbox. Stimuli were white capital letters: R, H, L, or F, shown on a black background. One event of the stimulus is a string of five letters: four identical letters, "flankers" flanking one non-identical "target" letter (Figure 19). The target letter was placed randomly in the $2^{\text {nd }}, 3^{\text {rd }}$, or $4^{\text {th }}$ position in the string. Subjects were given one response box in each hand and were instructed to press the left response button when the target letter is "L" or " $\mathrm{F}$ ", or alternatively the right response button when the target letter is " $\mathrm{R}$ " or " $\mathrm{H}$ ". The stimulus is congruent when the target codes for the same response as the flankers, or alternatively, is incongruent when the target codes for the opposite response as the flankers (Figure 19).

There were 80 events in one run (including a 12-s initial baseline): 60 stimuli, and 20 randomly occurring null events which have no letter stimuli, and were added into the paradigm in order to match the duration of the CPT paradigm. The duration of one event of the stimulus was $500 \mathrm{~ms}$ and the interstimulus interval was jittered randomly by an integer between $2 \mathrm{~s}$ and $10 \mathrm{~s}$, with an average of $6 \mathrm{~s}$. One Flanker run lasted 8.13 minutes (244 volumes). Throughout the presentation of those 60 stimuli, occurrence of a congruent or an incongruent stimuli event has a $50 \%$ probability each. Out of those, 50\% probability coded for a left button press and $50 \%$ probability coded for a right button press.
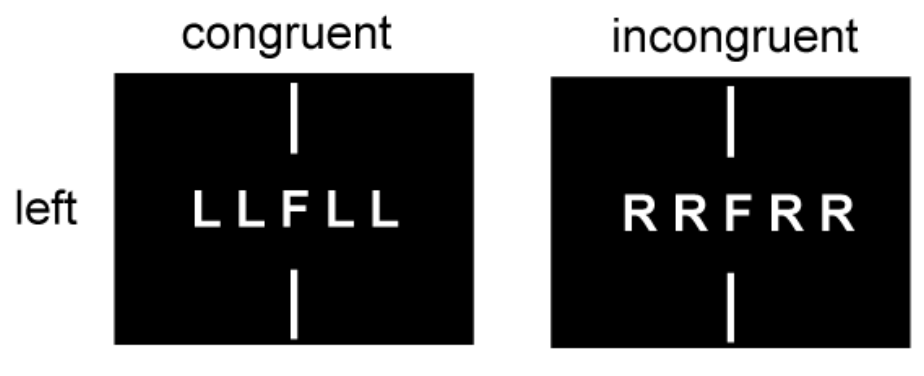

Figure 19: Example of the Flanker stimuli. Subjects were instructed to press the left button if the target letter is " $\mathrm{L}$ " or " $\mathrm{F}$ "; or alternatively the right button if the target letter is " $R$ " or " $\mathrm{H}$ ". 
Subjects were instructed to be as fast and as accurate as possible. Feedback on the results (average reaction time and accuracy) was shown on the screen at the end of the run. In the pre-training session, before performing the task, a simulation of the Flanker task was shown to the subjects outside the scanner to help them understand the task. Online rt-fMRI monitoring of the BOLD was not performed with rt-fMRI using TBV because unlike CPT that used pre-determined stimuli, the Flanker stimuli for each subject were random and determined on the fly - thus incompatible with TBV's requirements. Nevertheless, subjects' responses were monitored real-time in MATLAB.

\subsubsection{Behavioral analyses}

Each subject's log files from the behavioral tasks were processed offline in MATLAB and SPSS. Reaction time and accuracy were analyzed individually for all subjects, and subsequently group analysis were performed for Learners and Non-Learners (criteria defined in Section 3.2.9.1), independent of the Serial or Parallel training paradigm, i.e. the Learners from the ST group and PT group were combined and compared with the Non-Learners across both training paradigms.

Reaction time for CPT and Flanker was determined from the onset of stimulus to the time of the button press. Accuracy for CPT was defined as the percentage of the sum of non-responses to non-target (out of 64) and correct responses to target (out of 16). Overall accuracy for Flanker was determined by percentage of correct responses to the stimuli (out of 60). Accuracy for congruent or incongruent stimuli was the percentage of correct responses to congruent stimuli or incongruent stimuli respectively.

Reaction time and accuracy in all subjects were analyzed using 3-way mixed ANOVAs on the factors Time (Post vs. Pre), Group (trained vs. control), and PARADIGM (Serial vs. Parallel). In the group of trained subject, reaction time and accuracy of Learners and Non-Learners were analyzed using 2-way mixed ANOVA on the factors Time (Post vs. Pre) and LEarning Status (Learner vs. Non-Learner).

Additionally for the Flanker task, the reaction time and accuracy for congruent and incongruent condition were also examined using a 3-way mixed ANOVA with factors Congruency (Incongruent vs. Congruent), Time (Post vs. Pre), and LEARNING STATUS (Learner vs. Non-Learner). 


\subsection{6 fMRI whole-brain analyses}

For a description of general fMRI analysis methods, please refer to Section 2.2.5.1.

\subsubsection{CPT}

The fMRI data was modeled in the first-level GLM by two conditions: the period of 46 s time interval following all cues (letter 0 ) and 4-6 s interval following all distractors (letter H) (Luetcke et al., 2009). The contrast was cue > distractor, representing the cognitive process of anticipation. Brain activity associated with anticipation in the CPT was investigated by 2-way mixed ANOVA on the first level GLM contrast cue > distractor with factors Time (Pre vs. Post) and LEARning STATUS (Learner vs. Non-Learner, Learner vs. Control, and Non-Learner vs. Control). Additionally, post-hoc one-sample t-test was performed on the first level GLM contrast cue $>$ distractor separately for pre and post, in each learning status group.

\subsubsection{Flanker task}

The data was modeled in the first-level GLM by two conditions: incongruent and congruent. The contrast was incongruent $>$ congruent, representing the cognitive process of conflict resolution. Brain activity associated with conflict resolution in Flanker task was investigated by 2-way mixed ANOVA on the first level GLM contrast incongruent $>$ congruent with factors TIME (Pre vs. Post) and LEARNing STATUS (Learner vs. Non-Learner, Learner vs. Control, and Non-Learner vs. Control). 


\subsection{Results}

\subsubsection{Behavioral analyses}

\subsubsection{CPT reaction time}

Reaction times of subjects of the trained and control group in the CPT are shown in Figure 20. The 3-way ANOVA on the reaction time revealed a significant main effect of Time $\left(F_{1,26}=5.903, p=0.022\right)$. There is a difference in the mean reaction time across time, where the reaction time was lower at post-training ( $\mathrm{M}=474 \pm 20 \mathrm{~ms}$ SEM) than at pre-training ( $M=517 \pm 25 \mathrm{~ms}$ SEM). There is no main effect of neither GROUP nor PARADIGM; therefore there is no difference between Trained (PT, ST) and Control (PC, SC) groups, or between Serial (ST, SC) and Parallel paradigm (PT, PC). In addition, there is no significant interaction between any of the factors.

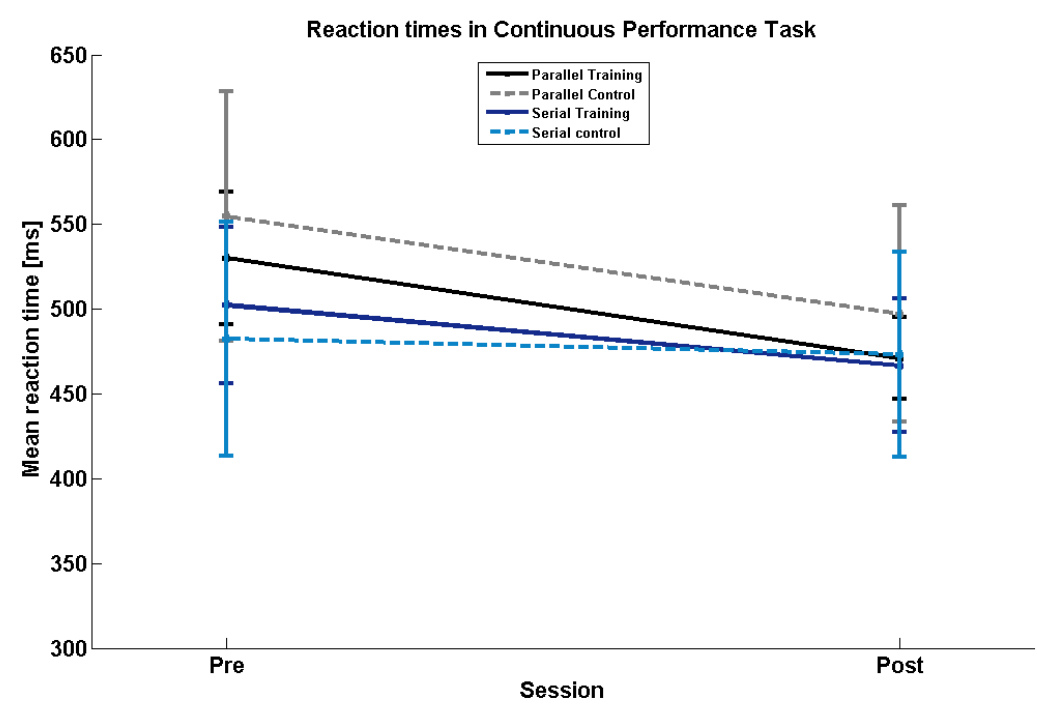

Figure 20: Reaction times of PT, PC, ST and SC groups in the CPT.

Reaction times of the Learners and Non-Learners in the CPT are shown in Figure 21. The 2-way ANOVA on the reaction time also revealed a significant main effect of TIME $\left(\mathrm{F}_{1,18}=5.386, \mathrm{p}=0.032\right)$, with a slower reaction time at pre-training $(\mathrm{M}=512 \pm$ $24.6 \mathrm{~ms}$, SEM) compared to post-training ( $\mathrm{M}=471 \pm 27.6 \mathrm{~ms}$ SEM). However there is no significant main effect of LEARNING STATUS. There is also no significant interaction between the two factors. 


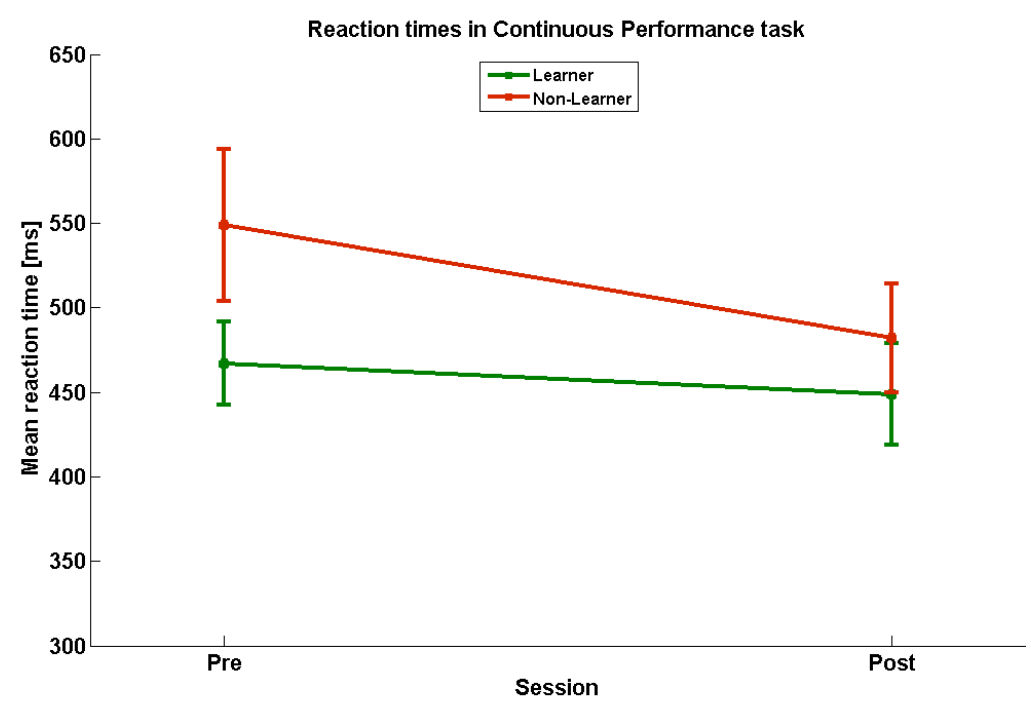

Figure 21: Reaction times of Learners and Non-Learners in the CPT

\subsubsection{CPT accuracy}

Accuracy values of the trained and control group in the CPT are shown in Figure 22. The accuracy was generally very high (pre-training: $\mathrm{M}=98.8 \pm 0.38 \%$ SEM; posttraining: $\mathrm{M}=99.2 \pm 0.25 \%$ SEM). The 3-way ANOVA revealed no significant effects, neither in TIME, PARADIGM or GROUP, nor any significant interactions.

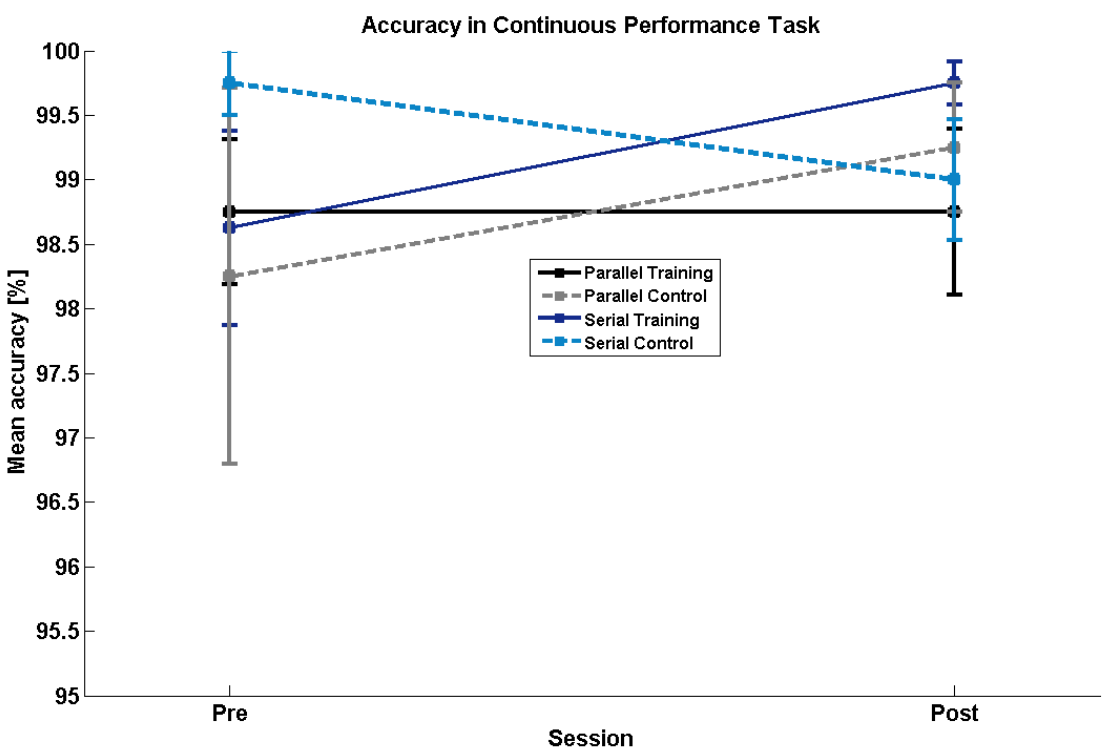

Figure 22: Accuracy of the PT, PC, ST and SC group in the CPT. 
Accuracy values of the group of Learners and Non-Learners in the CPT are shown in Figure 23. On average, the accuracy in CPT for both the Learners and Non-Learners was high, (pre-training: $M=98.9 \pm 0.5 \%$ SEM; post-training: $M=99.1 \pm 0.4 \%$ SEM). The 2-way ANOVA revealed no significant effects, neither in TimE or LEARNing STATUS, nor in the interaction between the two factors.

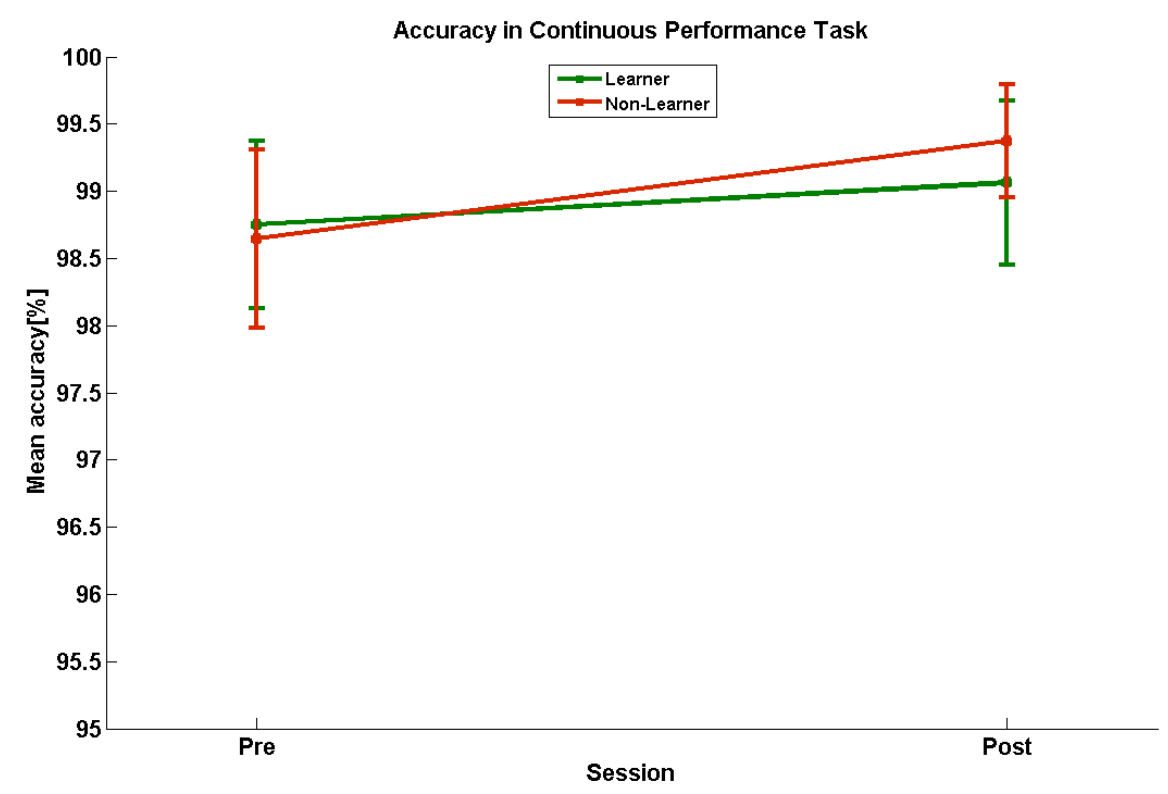

Figure 23: Accuracy of the learners and non-Learners group in the CPT.

\subsubsection{Flanker task reaction time}

Reaction time of the trained and control group in the Flanker task is shown in Figure 24. The 3-way ANOVA showed that there is a significant main effect of TIME $\left(\mathrm{F}_{1,26}=7.616, \mathrm{p}=0.010\right)$, where mean reaction time at post-training $(\mathrm{M}=1067 \pm 27$ ms SEM) was lower than that at pre-training ( $M=1137 \pm 37 \mathrm{~ms}$ SEM). Each of the groups was faster in the post-training than in the pre-training. No additional main effect of GROUP or PARADIGM, meaning that that there was no difference between trained (PT, ST) and control (PC, SC) groups; or between Serial (ST, SC) and Parallel paradigm (PT, PC). In addition, there was no significant interaction between any of the factors. 


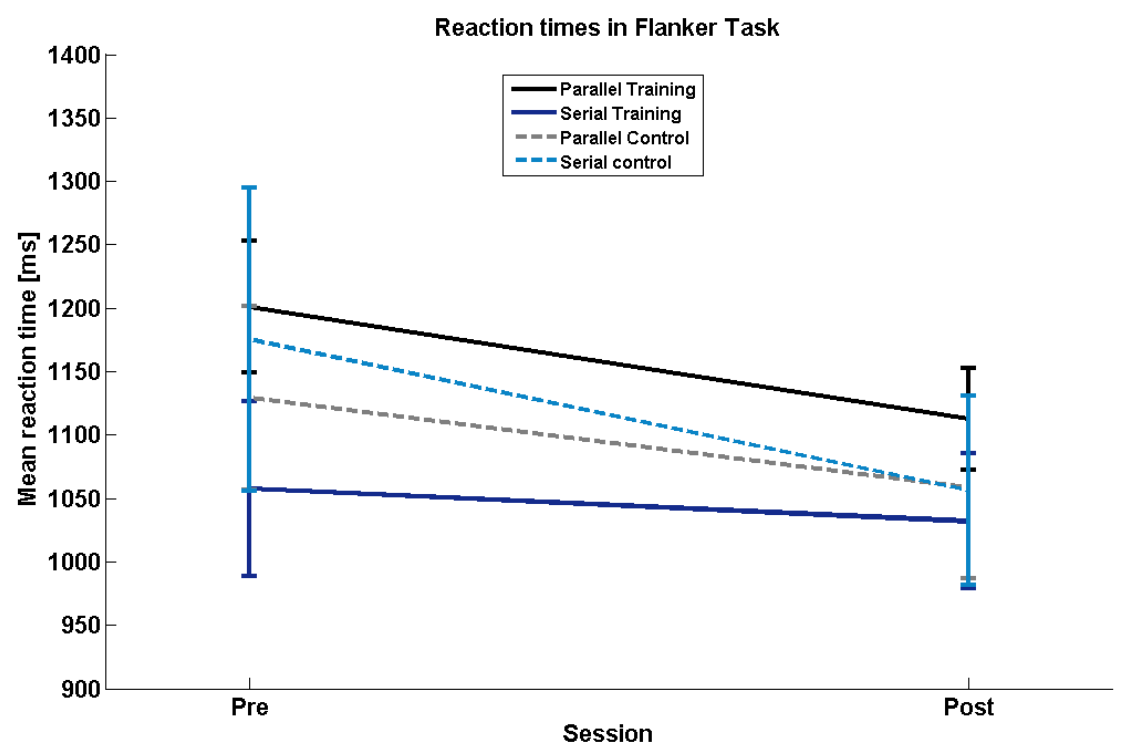

Figure 24: Reaction time of the PT, ST, PC, and SC groups in the Flanker task

Reaction time of the Learners and Non-Learners in the Flanker task is shown in Figure 25. The 2-way ANOVA on reaction time showed a significant main effect of LEARNING STATUS $\left(\mathrm{F}_{1,18}=4.795, \mathrm{p}=0.042\right)$ where the Learners on average were faster compared to the Non-Learners (Learners: $\mathrm{M}=1012.2 \pm 34.29$ ms SEM; NonLearners: $\mathrm{M}=1159.9 \pm 36.79 \mathrm{~ms}$ SEM).

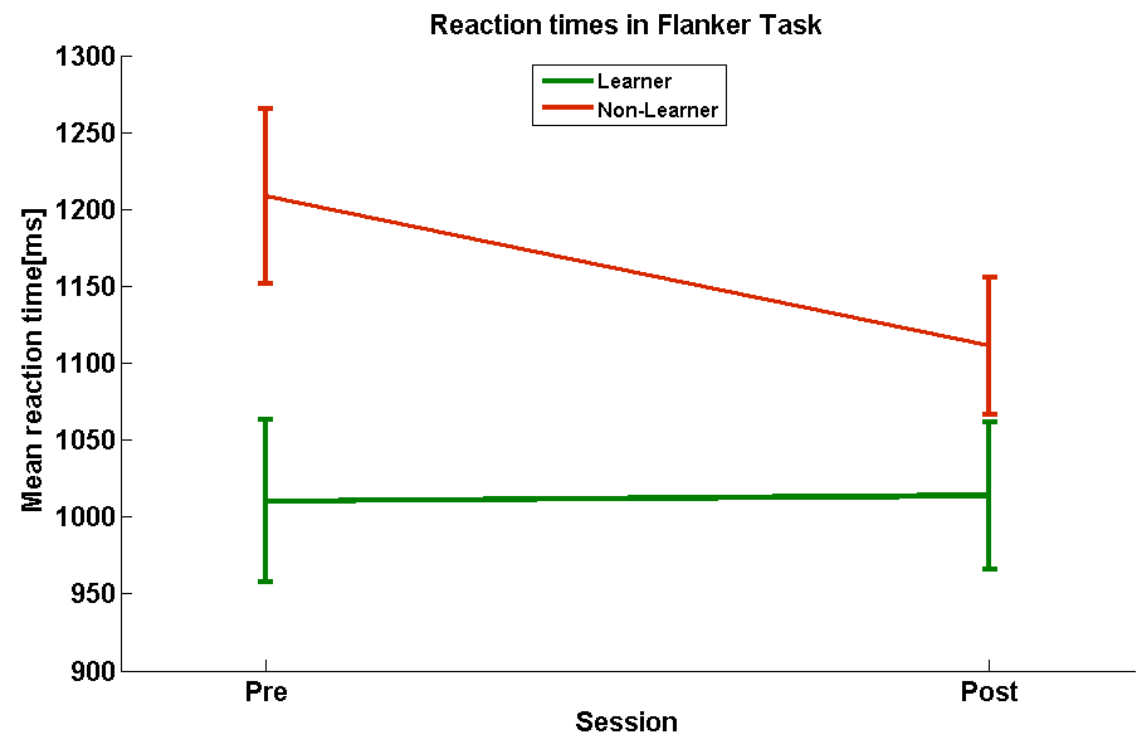

Figure 25: Reaction time of Learners and Non-Learners in the Flanker task 
This study also asked if there were any differences between Learners and NonLearners reflected in conflict resolution the Flanker task before and after NF training. For this, the additional factor of congruency in the 3-way ANOVA was investigated, and a LEARNING STATUS $\times$ CONGRUENCY $\times$ TIME interaction was expected. Figure 26 shows the effect of congruency of the Flanker stimuli on reaction times in Flanker task. The 3-way ANOVA on reaction time showed a significant main effect of Congruency $\left(\mathrm{F}_{1,18}=19.07, \mathrm{p}<0.001\right)$. The incongruent condition, independent of learning status and time, elicited slower reaction times. There was also a significant main effect of LEARNING STATUS $\left(\mathrm{F}_{1,18}=4.78, \mathrm{p}=0.042\right)$ in which Learners were generally faster than Non-Learners, regardless of congruency condition. Additionally the interaction between TIME $\times$ CONGRUENCY is significant, $\left(F_{1,18}=7.453, p=0.014\right)$ where regardless of learning status, the decrease in reaction time across sessions was larger in the congruent condition than the incongruent condition.

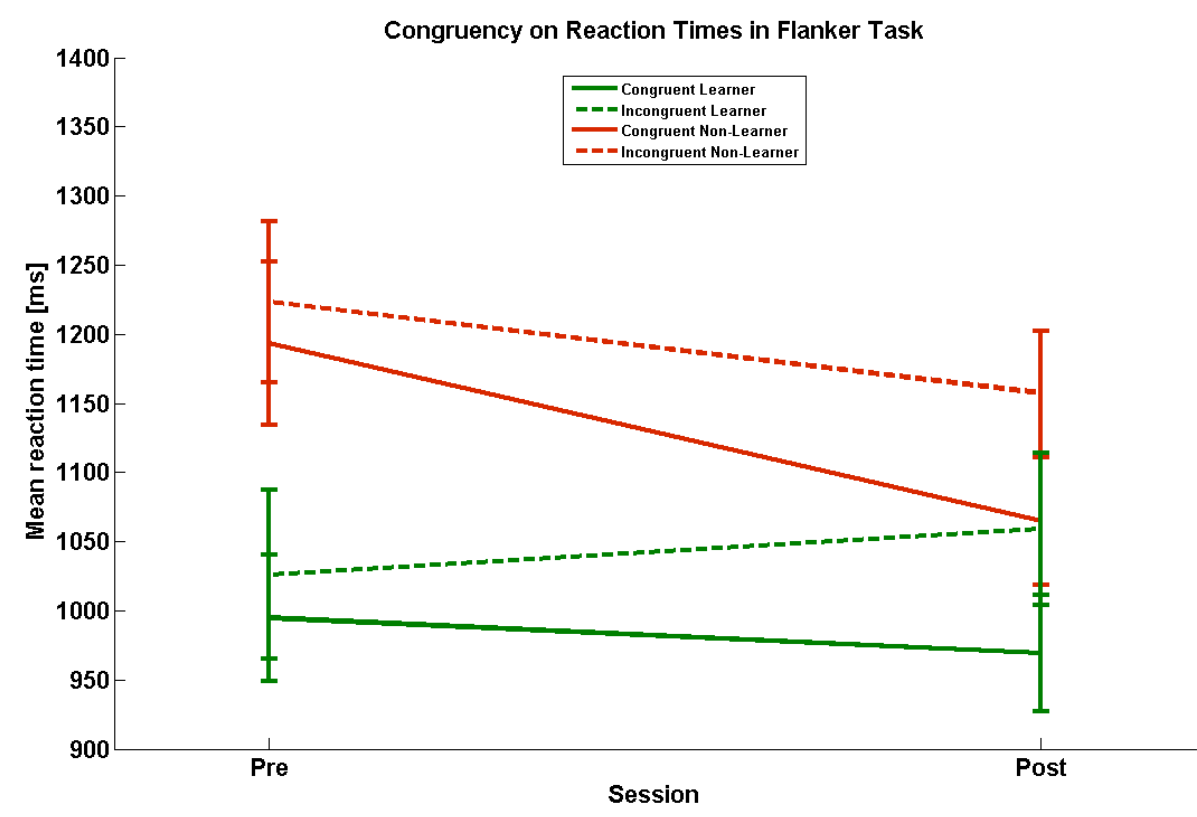

Figure 26: Reaction time in different conditions of congruency in Flanker task

\subsubsection{Flanker task accuracy}

Accuracy values of the trained and control groups in the Flanker task are shown in Figure 27. On average the accuracy in all groups are high $(\mathrm{M}=94.24 \pm 0.59 \% \mathrm{SEM})$. The 3-way ANOVA revealed no significant main effect of TIME, PARADIGM or GRouP. Additionally there were also no significant interactions between any of those factors. 


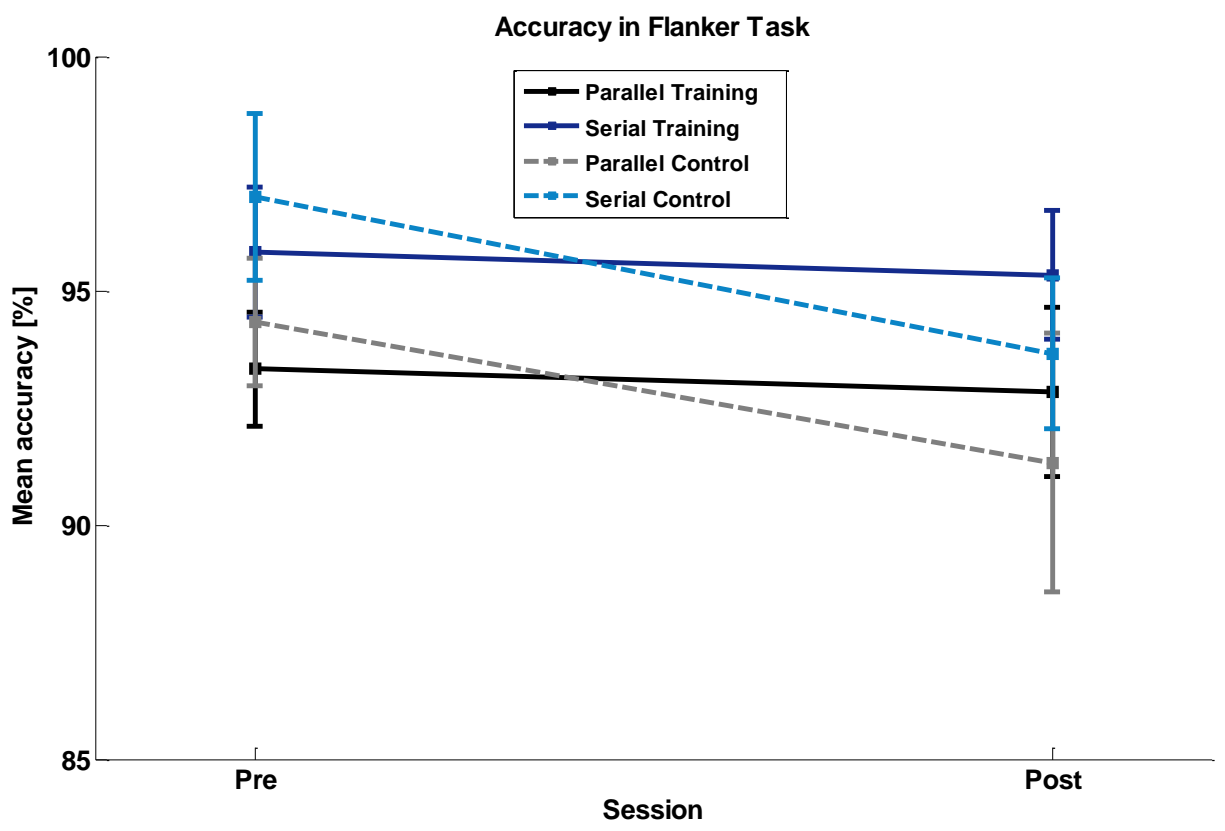

Figure 27: Accuracy in PT, ST, PC, and SC groups in the Flanker task

Accuracy values of the groups of Learners and Non-Learners in the Flanker task are shown in Figure 28. The 2-way ANOVA revealed no significant main effects of LEARNing STATUS or Time, and also no significant interaction between the two factors. Both groups performed the Flanker task on the same accuracy level.

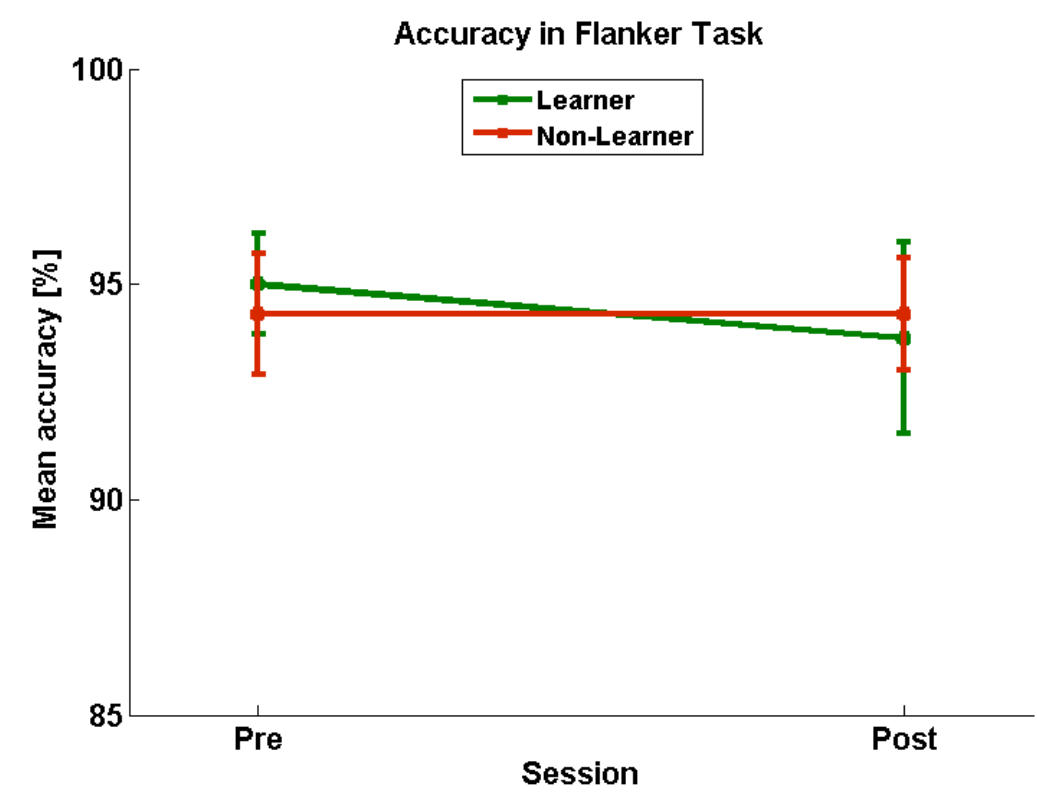

Figure 28: Accuracy in Learners and Non-Learners in the Flanker task 
Congruency was also analyzed within the group of Learners and Non-Learners. Figure 29 shows the congruency effect of the Flanker stimuli on accuracy in Flanker task. The 3-way ANOVA showed a significant main effect of CoNGRUENCY $\left(\mathrm{F}_{1,18}=\right.$ 10.11, $\mathrm{p}=0.005)$, where the congruent stimuli elicited higher accuracy than incongruent stimuli, regardless of time and learning status. There was no significant main effect of TIME or LEARNing STATUS; and no interaction between any of the factors.

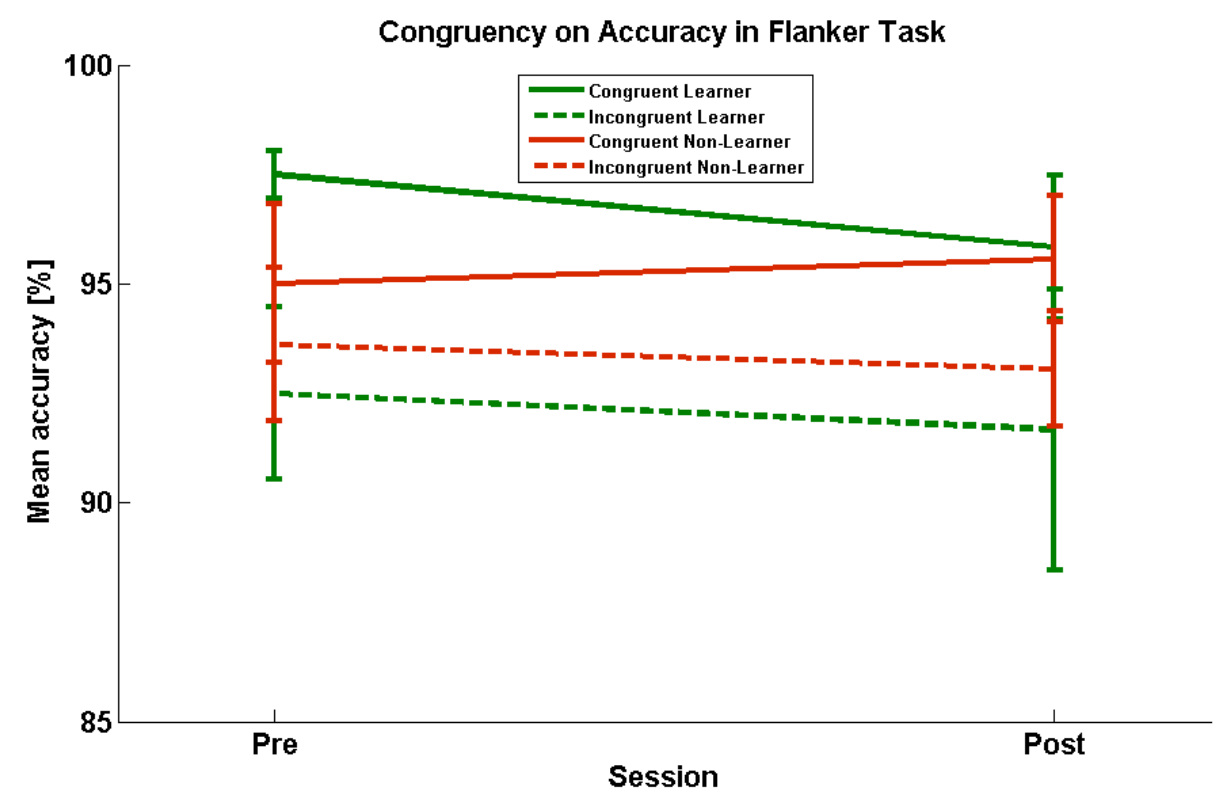

Figure 29: Accuracy in different conditions of congruency in the Flanker task

\subsection{2 fMRI whole-brain analyses}

Whole-brain fMRI analysis was performed to look for changes on the neuronal level in the behavioral transfer session, brought about by NF training of aMCC selfregulation.

\subsubsection{CPT}

In the CPT, comparison between Serial and Parallel paradigms across times did not show significant difference in brain activation. Therefore, similar to the NF analysis, a comparison between the different learning statuses was performed. Initial analyses showed no difference in brain activation across the transfer sessions (Pre vs. Post) in the Learners vs. Control, Learner vs. Non-Learner, and Non-Learner vs. 
Control comparison. Subsequent analysis investigated each group at each time point separately. Brain activations in Learners, Controls, and Non-Learners, during the pre- as well as the post-training sessions are shown in Figure 30. Bilateral insula, aMCC, SMA, and left SMC were activated in each of the groups, during both time points. This explains the lack of difference in brain activation in the post-pre comparison.
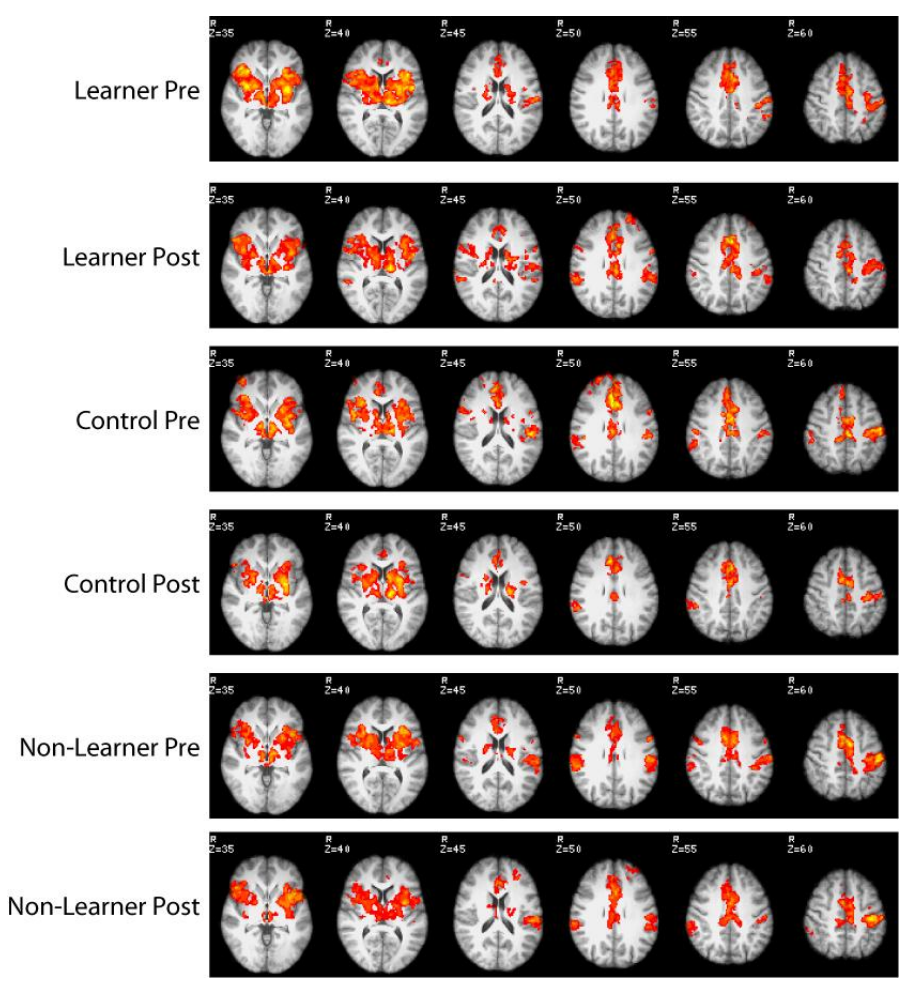

Figure 30: Brain areas activated in Learners, Controls, and Non-Learners in CPT during pre- and post- NF training. Activations in bilateral insula, basal ganglia, aMCC, SMA and left SMC can be seen. Images are thresholded at $z>2.3$, cluster significance threshold of $p=0.05$ (FWE corrected).

\subsubsection{Flanker task}

A comparison of brain activation changes across the pre-post transfer sessions and across the learning statuses and controls is shown in Figure 31 and Table 3. Compared to the controls, the Learners showed increase of brain activation in the right putamen and right pallidum from the pre- to post transfer session (Figure 31 A). There is no difference in brain activation from the pre- to post transfer session between Non-Learners and Controls (Figure 31 B). Compared to the Non-Learners, the Learners showed increased activity in the inferior frontal gyrus (IFG), putamen, pallidum, thalamus, precentral gyrus, postcentral gyrus, and middle frontal gyrus (Figure 31 C). Although increased activity was not seen in the aMCC in any of those 
three higher level comparisons, it was nevertheless more activated in the Learners group at post-training compared to pre-training transfer session (results not shown).

A. Learner $>$ Control

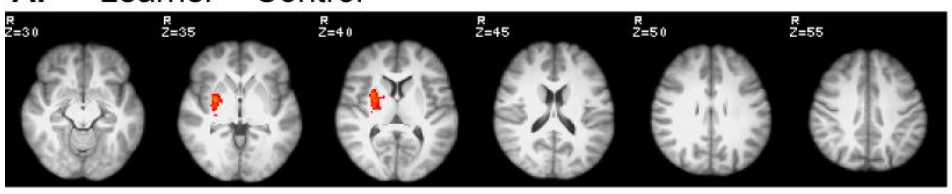

B. Non-Learner $>$ Control

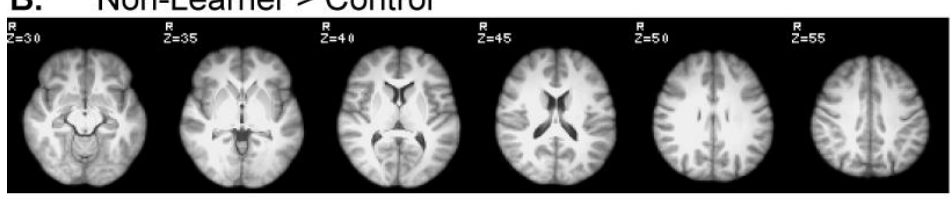

C. Learner $>$ Non-Learner

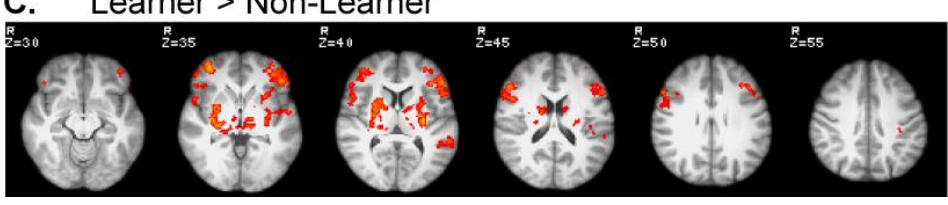

Figure 31: Brain areas significantly activated during incongruent $>$ congruent condition across transfer sessions in A). Learners compared to Controls, B). Non-Learners compared to Controls $\mathbf{C}$ ). Learners compared to Non-Learners in the Flanker task. Images are thresholded at $z>2.3$, cluster significance threshold of $p=0.05$ (FWE corrected). 
Table 3: Cluster maxima for changes of brain activation in conflict resolution in the Flanker task after NF training. Anatomical areas defined by Harvard-Oxford Cortical and Subcortical Maximum Probability Atlas.

\begin{tabular}{|c|c|c|c|c|c|}
\hline & \multirow{2}{*}{$\begin{array}{l}\text { Number of } \\
\text { voxels }\end{array}$} & \multicolumn{3}{|c|}{ MNI coordinates } & \multirow[t]{2}{*}{ Maximum z } \\
\hline & & $x$ & $\mathrm{Y}$ & $\mathrm{Z}$ & \\
\hline \multicolumn{6}{|l|}{ Learner > Control } \\
\hline Putamen R & 308 & 28 & -2 & -2 & 3.72 \\
\hline Pallidum R & 15 & 24 & -2 & -2 & 2.95 \\
\hline \multicolumn{6}{|l|}{ Non-Learner > Control } \\
\hline- & - & - & - & - & - \\
\hline \multicolumn{6}{|l|}{ Learner > Non-Learner } \\
\hline $\begin{array}{l}\text { Inferior Frontal Gyrus pars } \\
\text { triangularis L }\end{array}$ & 453 & -50 & 32 & 6 & 4.60 \\
\hline $\begin{array}{l}\text { Inferior Frontal Gyrus pars } \\
\text { triangularis R }\end{array}$ & 228 & 52 & 26 & 22 & 4.17 \\
\hline $\begin{array}{l}\text { Inferior Frontal Gyrus pars } \\
\text { opercularis R }\end{array}$ & 475 & 56 & 18 & 22 & 4.05 \\
\hline Putamen L & 412 & -28 & -18 & 6 & 4.53 \\
\hline Putamen R & 428 & 30 & -12 & 2 & 4.40 \\
\hline Thalamus L & 163 & -10 & -14 & 2 & 3.32 \\
\hline Thalamus $\mathrm{R}$ & 232 & 12 & -26 & 2 & 3.74 \\
\hline Precentral gyrus L & 527 & -34 & -14 & 46 & 3.69 \\
\hline Precentral gyrus $\mathrm{R}$ & 143 & 52 & 8 & 4 & 3.66 \\
\hline Postcentral gyrus L & 316 & -46 & -30 & 60 & 3.61 \\
\hline Middle Frontal gyrus L & 305 & -34 & 30 & 26 & 3.44 \\
\hline Middle Frontal gyrus $\mathrm{R}$ & 49 & 50 & 26 & 22 & 3.74 \\
\hline
\end{tabular}




\subsection{Discussion}

This study investigated effects of NF self-regulation of the aMCC on two behavioral tasks: the CPT and the Flanker task. For the CPT, the effects NF-related learning were not observed in either the behavioral measures or the whole-brain fMRI analysis of the post-pre changes. For the Flanker task, such effects could not be seen in the behavioral measures, but were observed in the whole-brain fMRI analysis.

\subsubsection{Generalization of NF training to the CPT}

Subjects generally exhibited high performance in the CPT in reaction time and accuracy. In measure of reaction time; all group regardless of trained or controls, paradigm, or learning status, performed faster in post-training compared to pretraining session. These results show that there is a practice effect in CPT. In measure of accuracy, there is no significant difference between any of the compared groups across time because all groups exhibited high accuracy (close to $100 \%$ ). Similarly, at the whole-brain level, no significant NF-related differences have been observed in the comparison between Learners, Non-Learners and Control groups. The brain areas that are activated are consistent with the activation observed in the same task that was also used as the functional localizer in this study (Section 2).

The high accuracy reached in the CPT in the pre-training measurements indicates a ceiling effect for that population of subjects, and improvement in the post-training measurements was not possible. CPT has been used mainly as a behavioral test for the clinical population with problems in attention, for example ADHD and schizophrenia patients (Riccio et al., 2002). In such populations, CPT is more sensitive to detect deficits in certain aspects of cognitive processes between patients and healthy control subjects. Furthermore, the CPT also has also been used in the clinical population more extensively with EEG (rather than fMRI) to study neuroelectric activities that can offer direct indication of the physiological mechanisms of sustained attention. Therefore, CPT-fMRI in its current form is not a suitable behavioral task to test generalization of rt-fMRI NF training of the aMCC in healthy subjects.

\subsubsection{Generalization of NF training to the Flanker task}

This study did not observe NF-related changes to the behavioral measures in the Flanker task. Subjects generally also exhibited high performance in the Flanker task 
in the overall reaction time and accuracy. However, the important cognitive processes to be examined further are conflict resolution and response inhibition. In the Flanker task, subjects need to resolve the conflict between the required response coded by the target letter, and alternative response coded by the conflicting flankers. In order to resolve this conflict, subjects need to inhibit a motor response (button press) if the target letter conflicts with the response coded by the flankers in the incongruent condition. In this task, one would expect a slower and less accurate response in the incongruent condition compared to the congruent condition due to the increase in cognitive processing to resolve the conflicting stimuli. In accordance to this, results of this current study demonstrated a congruency effect on the reaction time and accuracy. More importantly, it is expected to see increased ability to resolve conflict in the Learners more than the Non-Learners at post-training compared to pre-training i.e. an interaction between LEARNING STATUS $\times$ CONGRUENCY $\times$ TIME. However, behavioral changes possibly related to successful self-regulation of the aMCC were not seen in either the reaction time or the accuracy measures in the Flanker task, as there was no significant 3-way interaction. Nevertheless, in the reaction time measure, the Learners were generally faster in both congruent and incongruent conditions compared to the Non-Learners.

On the whole-brain level, our results suggest possible generalization effects of a successful NF training of the aMCC to the neural correlates of the Flanker task. Prominent activation in the inferior frontal gyrus (IFG) and the basal ganglia in the Flanker task is consistent with the role of IFG in response inhibition, and as part of the fronto-basal ganglia inhibition network (Verbruggen and Logan, 2008). It may be that the Learners showed better processing of these aspects. A behavioral task similar to the Flanker task involving resolution of congruent and incongruent conditions, called the Simon task (Peterson et al., 2002), has also been used to test generalization of learned regulation of the ACC (Mathiak et al., 2010). The authors however did not report behavioral measures nor increase in other brain areas apart from the ACC. However, it should be noted that it was a pilot study using one subject and a much shorter training duration. 


\subsection{Conclusions}

In conclusion, the Flanker task may be a better measure than CPT to investigate effects of learned self-regulation of the aMCC in the domain of cognitive control, at least on the whole-brain level. Effects of successful self-regulation of the aMCC may have generalized to the fronto-basal ganglia network, implicated in response inhibition - one of the cognitive processes involved in the Flanker task. This study also questions the usefulness of the CPT in its present form as a behavioral task to test learned regulation of the aMCC. 


\section{Summary}

\section{Summary}

This thesis deals with an extensive exploration of the use of rt-fMRI NF in cognition. Specifically, the work presented here comprises the major steps in the development of a suitable experimental framework for rt-fMRI NF in a cognitive brain region: defining a target region, defining a strategy and protocol for training the target region by NF, and testing the cognitive and behavioral effects of NF training.

\subsection{Defining the aMCC}

In the present study the aMCC was defined as a cognitive target region based on its function. This choice avoids a possible anatomical selection, because the aMCC anatomy exhibits considerable intersubject variability and because the main intention was to train specifically its cognitive control function. In the study described in Chapter 2 , the location and definition of the aMCC in each individual subject was achieved by probing the aMCC function through a behavioral test - the CPT. BOLD activation associated with the cognitive process of anticipation, which is expected to arise in the aMCC, was analyzed with two different methods, the classical GLM approach and the combination of ICA with GLM. The GLM method resulted in optimal localization of the aMCC in 7 out of 10 subjects, while the ICA-GLM method localized the aMCC robustly in all 10 subjects. The CPT with ICA-GLM analysis was then tested in an additional set of subjects, and it showed again its ability to localize and define the aMCC in all subjects. An additional focus in this chapter is the advantageous use of rt-fMRI to monitor the results of the fMRI measurement online. The present results strongly encourage real-time monitoring of the functional localizer scans for quality control reasons.

The results of Chapter 2 demonstrate that the CPT is a suitable cognitive paradigm for functional localization of the aMCC, and that ICA-GLM is a suitable analysis method for that purpose.

\subsection{Neurofeedback training of the aMCC}

Chapter 3 details the extensive rt-fMRI NF training on the aMCC, which had to meet various challenges arising from the latency of the NF signal, the processing of the feedback signal in parallel with the optimization of a self-regulation strategy, and the possible interference of these two processes in the aMCC. To resolve the described challenges, this work introduced a novel Serial NF paradigm, which temporally uncouples self-regulation and feedback appraisal. 


\section{Summary}

Healthy subjects were trained to learn how to self-regulate their aMCC with feedback information in $18 \mathrm{NF}$ training runs in 6 sessions spread over two weeks. Transfer runs without feedback information were performed before beginning and after the end of training. Two separate groups of 10 subjects each underwent NF training: one trained with the Serial paradigm, and the other with the conventional Parallel paradigm. Two separate control groups only performed the two transfer runs. Overall success in $\mathrm{NF}$ in trained subjects was defined as a larger increase of aMCC activity from the pre- to post-training transfer runs without the feedback than observed for controls. Efficiency during NF training was determined in a similar manner, defined as achieving a larger increase in aMCC activity in the training runs. Subjects answered questionnaires about the paradigm and their mental strategy.

Six out of 10 subjects in the Serial paradigm successfully learned self-regulation of the aMCC compared to only two out of 10 for the Parallel paradigm. These individuals, subsequently categorized as "Learners", showed higher change in their aMCC activity during the transfer task without feedback than subjects of the control group. Compared to Non-Learners, Learners also showed more efficiency in using the feedback during NF training. Whole-brain analysis of the transfer runs revealed that they also activated brain regions related to reward processing apart from the aMCC itself. Questionnaire analysis indicated that subjects of the Serial paradigm felt more in control of the NF task. The newly introduced temporal separation of the feedback appraisal from the selfregulation enables investigation of the brain activity associated with feedback appraisal. Feedback appraisal appeared to elicit lower aMCC activity than self-regulation, and there was no difference in aMCC activity between the Learners and Non-Learners. Feedback appraisal could further be characterized as a self-referential activity that engages the default mode network - a large-scale network of brain regions that constitutes an integrated system for self-related cognitive processes such as internal mentation.

Taken together, the findings in Chapter 3 demonstrate that the Serial paradigm emerges as a promising new tool in facilitating learning in rt-fMRI NF. In addition, it allows for a separate investigation of the cognitive process of feedback appraisal.

\subsection{Effect of neurofeedback training of the aMCC}

Chapter 4 employed two behavioral tests, the CPT and Flanker task, as measures to investigate the effects of successful self-regulation of the aMCC on the processes of 
anticipation and conflict resolution, respectively. The behavioral tests were incorporated in the pre-training and post-training session.

On the behavioral level, the effects of successful training of the aMCC with rt-fMRI NF did not appear to cause significant changes in behavioral measures, neither for the CPT nor the Flanker task. On the whole brain level, fMRI did not reveal effects of successful aMCC NF training in the CPT, which was in contrast to the positive results for the Flanker task. In the latter case, learners demonstrated increased activation in the inferior frontal gyrus and basal ganglia in the Flanker task after successful selfregulation of the aMCC, suggesting increased involvement of the fronto-basal ganglia network implicated in response inhibition - a process that is needed to resolve the conflicting Flanker stimuli.

To conclude, on the whole-brain level, the findings in Chapter 4 showed a possible effect of successful self-regulation of the aMCC on the cognitive process of conflict resolution in the Flanker task. 


\section{Outlook}

\subsection{Neurofeedback and cognitive networks}

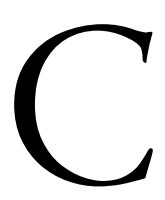

ognitive processes usually include coordinated activity of several brain regions, thus it is always worthwhile to look beyond a single brain region. Studies in rt-fMRI NF have recently made first advances to progress from targeting circumscribed brain areas to implementing connectivity analysis either at the level of training, e.g. regulating connectivity between multiple brain areas (Koush et al., 2013) or at the level of transfer, e.g. testing the effect of successful self-regulation on brain connectivity. While the former aspect is technically more challenging (the study referenced above was a proof-of-concept study), the latter aspect has been more actively explored recently. An rt-fMRI NF of the insula in schizophrenic patients showed increased effective connectivity between the insula and brain areas involved in emotional network like amygdala and medial pre-frontal cortex (Ruiz et al., 2013). Another rt-fMRI NF study of the visual cortex showed increase effective connectivity between the trained visual ROI and the superior parietal lobule contralateral to the trained ROI (Scharnowski et al., 2014). An EEG-NF study of alpha rhythm self- regulation has discovered functional connectivity changes in the salience network (SN) even after one training session (Ros et al., 2013). These developments are important as they not only contribute to the understanding of the basic principles of self-regulation of circumscribed brain areas, but further explore their influence and role in various brain networks. In addition, they are also promising in clinical neurosciences because more effective and personalized therapy can be designed if the basic mechanisms of disorders are better understood.

Applying the developments in connectivity to the data presented in this thesis would be a worthwhile step forward. The focus would be on brain connectivity examined within the NF training session or in the pre-post transfer session. Functional connectivity analysis using Psychophysiological Interactions (PPI) within NF training could reveal areas whose connectivity with the aMCC changes depending on whether or not the subject is up-regulating. An interesting connectivity aspect to investigate further is possible NF-induced changes in intrinsic connectivity networks (ICNs) - large-scale network of interconnected brain regions observed in subjects during rest (Menon, 
2011). There are three distinct cognitive ICN: the salience network (SN) - a large-scale brain network involved in detecting the most important external stimuli and internal events; the central executive network (CEN) - a network involved in working memory, attention and decision making in goal-directed behavior; and the default mode network (DMN) - a network involved in self-referential processes. I speculate that successful self-regulation of the aMCC could bring about connectivity changes particularly within the SN. This is because aMCC, together with the anterior insula are nodes of the SN. This aspect can be investigated using model-free connectivity analysis using group-ICA and dual regression. Moreover, the SN has a role in switching between the CEN and the DMN (Sridharan et al., 2008). It could mediate continuous switching between the selfregulation phase, which is more goal-directed, and the feedback appraisal phase which is more self-referential. Investigating these speculations would need model-based connectivity analyses that probe into the directionality of networks like Granger Causality or Dynamic Causal Modelling.

As for further experiments, in my opinion, it is most important to extend the current NF experiment to the SMC, a non-cognitive brain area which is well-studied in rt-fMRI NF. The Serial paradigm would be compared to the conventional Parallel paradigm to see how much the former would help facilitating self-regulation of a noncognitive area. I speculate that the Serial paradigm would show more efficacy in rt-fMRI $\mathrm{NF}$ on the SMC because the core concepts of the Serial paradigm in reducing the cognitive load and explicit temporal contiguity would still hold; and in addition, mental strategies (e.g. motor imagery) to voluntarily activate the SMC are easier to perform. At the whole-brain level, it would be interesting to see if the involvement of the reward system observed in learning self-regulation of the aMCC, and the DMN in feedback appraisal, are general to NF of any brain area or only specific to NF of the aMCC.

Although the complexity in rt-fMRI NF experiments are growing, the fundamental questions about learning self-regulation of a single, circumscribed brain region are still relevant and worth exploring. It is my hope that understanding this small piece of the puzzle contributes to the big picture of understanding brain function in basic neuroscience. 


\section{References}

Amiez, C., Neveu, R., Warrot, D., Petrides, M., Knoblauch, K., and Procyk, E. (2013). The Location of Feedback-Related Activity in the Midcingulate Cortex Is Predicted by Local Morphology. J Neurosci 33, 2217-28.

Andersson, J., Jenkinson, M., and Smith, S. (2007). "Non-linear registration a.k.a Spatial Normalisation". (Oxford, England).

Andrews-Hanna, J. (2012). The brain's default network and its adaptive role in internal mentation. The Neuroscientist 18, 251-70.

Auer, T., and Frahm, J. (2011). Confounding factors in neurofeedback training based on fMRI of motor imagery. Neurosci Lett 500, Supplement, e32.

Beckmann, C.F. (2012). Modelling with independent components. Neuroimage 62, 891901.

Beckmann, C.F., and Smith, S.A. (2004). Probabilistic independent component analysis for functional magnetic resonance imaging. IEEE T Med Imaging 23, 137-52.

Beckmann, C.F., and Smith, S.M. 2013. Probabilistic Independent Component Analysis

for Functional Magnetic Resonance Imaging. FMRIB Technical Report TR02CB1 [Online]. [Accessed 7 July 2014].

Berman, B.D., Horovitz, S.G., Venkataraman, G., and Hallett, M. (2012). Self-modulation of primary motor cortex activity with motor and motor imagery tasks using realtime fMRI-based neurofeedback. Neuroimage 59, 917-25.

Birbaumer, N., Ruiz, S., and Sitaram, R. (2013). Learned regulation of brain metabolism. Trends Cogn Sci 17, 295-302.

Bradley, M., and Lang, P. (1994). Measuring emotion: the Self-Assessment Manikin and the Semantic Differential. J Behav. Ther. Exp. Psychiatry 25, 49-59.

Brandmeyer, T., and Delorme, A. (2013). Meditation and neurofeedback. Frontiers in psychology 4, 688.

Bray, S., Shimojo, S., and O' Doherty, J. (2007). Direct instrumental conditioning of neural activity using functional magnetic resonance imaging-derived reward feedback. $J$ Neurosci 27, 7498-507.

Caria, A., Sitaram, R., Veit, R., Begliomini, C., and Birbaumer, N. (2010). Volitional Control of Anterior Insula Activity Modulates the Response to Aversive Stimuli. A RealTime Functional Magnetic Resonance Imaging Study. Biological Psychiatry 68, 425-32.

Caria, A., Veit, R., Sitaram, R., Lotze, M., Weiskopf, N., Grodd, W., and Birbaumer, N. (2007). Regulation of anterior insular cortex activity using real-time fMRI. Neuroimage 35, 1238-46.

Caulo, M., Esposito, R., Mantini, D., Briganti, C., Sestieri, C., Mattei, P.A., Colosimo, C., Romani, G.L., and Tartaro, A. (2010). Comparison of hypothesis- and a novel hybrid data/hypothesis-driven method of functional MR imaging analysis in patients with brain gliomas. AJNR Am J Neuroradiol. 32, 1056-64.

Chiew, M., Laconte, S.M., and Graham, S.J. (2012). Investigation of fMRI neurofeedback of differential primary motor cortex activity using kinesthetic motor imagery. Neuroimage 61, 21-31.

Cox, R.W., Jesmanowicz, A., Hyde, J.S. (1995). Real-time functional magnetic resonance imaging. Magn Reson Med. 33, 230-6. 
Cutini, S., and Brigadoi, S. (2014). Unleashing the future potential of functional nearinfrared spectroscopy in brain sciences. J Neurosci Methods 232, 152-6.

Decharms, R.C., Christoff, K., Glover, G.H., Pauly, J.M., Whitfield, S., and Gabrieli, J.D.E. (2004). Learned regulation of spatially localized brain activation using real-time fMRI. Neuroimage 21, 436-43.

Decharms, R.C., Maeda, F., Glover, G.H., Ludlow, D., Pauly, J.M., Soneji, D., Gabrieli, J.D.E., and Mackey, S.C. (2005). Control over brain activation and pain learned by using real-time functional MRI. Proc. Natl. Acad. Sci. U.S.A 102, 18626-31.

Delgado, M.R., Miller, M.M., Inati, S., and Phelps, E.A. (2005). An fMRI study of rewardrelated probability learning. Neuroimage 24, 862-73.

Dewiputri, W.I., and Auer, T. (2013). Functional magnetic resonance imaging neurofeedback: implementations and applications. Malaysian J Med Sci 20, 5-15.

Eriksen, B.A., and Eriksen, C.W. (1974). Effects of Noise Letters Upon Identification of a Target Letter in a Nonsearch Task. Percept Psychophys. 16, 143-9.

Fox, M.D., and Raichle, M.E. (2007). Spontaneous fluctuations in brain activity observed with functional magnetic resonance imaging. Nat Rev Neurosci 8, 700-11.

Gazzaniga, M.S., Ivry, R.B., and Mangun, G.R. (2009). Cognitive Neuroscience: The Biology of the Mind. New York: W.W Norton \& Company.

Greer, S.M., Trujillo, A.J., Glover, G.H., and Knutson, B. (2014). Control of nucleus accumbens activity with neurofeedback. Neuroimage 96, 237-44.

Greville, W.J., Cassar, A. A., Johansen, M.K., Buehner, M.J. (2013). Structural awareness mitigates the effect of delay in human causal learning. Mem Cogn. 41, 904-916

Greville, W., and Buehner, M. (2010). Temporal predictability facilitates causal learning. J Exp Psychol Gen. 139, 756-71.

Gröne, M., Dyck, M., Koush, Y., Bergert, S., Mathiak, K.A., Alawi, E.M., Elliott, M., and Mathiak, K. (2014). Upregulation of the Rostral Anterior Cingulate Cortex can Alter the Perception of Emotions: fMRI-Based Neurofeedback at 3 and 7 T. Brain Topogr., 1-11.

Haber, S.N., and Calzavara, R. (2009). The cortico-basal ganglia integrative network: The role of the thalamus. Brain Res Bull 78, 69-74.

Hamilton, J.P., Glover, G.H., Hsu, J.-J., Johnson, R.F., and Gotlib, I.H. (2011). Modulation of subgenual anterior cingulate cortex activity with real-time neurofeedback. Hum Brain Mapp. 32, 22-31.

Heinrich, H., Gevensleben, H., Freisleder, F.J., Moll, G.H., and Rothenberger, A. (2004). Training of slow cortical potentials in attention-deficit/hyperactivity disorder: evidence for positive behavioral and neurophysiological effects. Biological Psychiatry 55, 772-5.

Hoffstaedter, F., Grefkes, C., Caspers, S., Roski, C., Palomero-Gallagher, N., Laird, A.R., Fox, P.T., and Eickhoff, S.B. (2013). The role of anterior midcingulate cortex in cognitive motor control. Hum Brain Mapp. 35, 2741-53.

Hyvärinen, A. (1999). Fast and Robust Fixed-Point Algorithms for Independent Component Analysis. IEEE Transactions on Neural Networks 10, 626-34.

Jenkinson, M. (2003). Fast, automated, N-dimensional phase-unwrapping algorithm. Magn Reson Med. 49, 193-7.

Jenkinson, M., Bannister, P., Brady, M., and Smith, S. (2002). Improved optimization for the robust and accurate linear registration and motion correction of brain images. Neuroimage 17, 825-41.

Jenkinson, M., and Smith, S.M. (2001). A global optimisation method for robust affine registration of brain images. Med Image Anal. 5, 143-56. 
Johnson, K.A., Hartwell, K., Lematty, T., Borckardt, J., Morgan, P.S., Govindarajan, K., Brady, K., and George, M.S. (2012). Intermittent "real-time" fMRI feedback is superior to continuous presentation for a motor imagery task: a pilot study. $J$ Neuroimaging 22, 58-66.

Kelly Jr, R.E., Alexopoulos, G.S., Wang, Z., Gunning, F.M., Murphy, C.F., Morimoto, S.S., Kanellopoulos, D., Jia, Z., Lim, K.O., and Hoptman, M.J. (2010). Visual inspection of independent components: Defining a procedure for artifact removal from fMRI data. J Neurosci Methods 189, 233-45.

Kober, S.E., Witte, M., Ninaus, M., Neuper, C., and Wood, G. (2013). Learning to modulate one's own brain activity: The effect of spontaneous mental strategies. Front Hum Neurosci 7.

Koralek, A.C., Jin, X., Long Ii, J.D., Costa, R.M., and Carmena, J.M. (2012). Corticostriatal plasticity is necessary for learning intentional neuroprosthetic skills. Nature 483, 331-5.

Koush, Y., Rosa, M.J., Robineau, F., Heinen, K., W Rieger, S., Weiskopf, N., Vuilleumier, P., Van De Ville, D., and Scharnowski, F. (2013). Connectivity-based neurofeedback: dynamic causal modeling for real-time fMRI. Neuroimage 81, 422-30.

Krigolson, O.E., Heinekey, H., Kent, C.M., and Handy, T.C. (2012). Cognitive load impacts error evaluation within medial-frontal cortex. Brain Research 1430, 62-7.

Lawrence, E.J., Su, L., Barker, G.J., Medford, N., Dalton, J., Williams, S.C.R., Birbaumer, N., Veit, R., Ranganatha, S., Bodurka, J., Brammer, M., Giampietro, V., and David, A.S. (2014). Self-regulation of the anterior insula: Reinforcement learning using realtime fMRI neurofeedback. Neuroimage 88, 113-24.

Lee, S., Ruiz, S., Caria, A., Veit, R., Birbaumer, N., and Sitaram, R. (2011). Detection of Cerebral Reorganization Induced by Real-time fMRI Neurofeedback Training of Insula Activation: A Multivariate Investigation Neurorehabil Neural Repair. 25, 259-67.

Liljeholm, M., and O'Doherty, J.P. (2012). Contributions of the striatum to learning, motivation, and performance: an associative account. Trends Cogn Sci 16, 467-75.

Linden, D.E.J., Habes, I., Johnston, S.J., Linden, S., Tatineni, R., Subramanian, L., Sorger, B., Healy, D., and Goebel, R. (2012). Real-Time Self-Regulation of Emotion Networks in Patients with Depression. PLoS One 7.

Luetcke, H., and Frahm, J. (2008). Lateralized anterior cingulate function during error processing and conflict monitoring as revealed by high-resolution fMRI. Cerebral Cortex 18, 508-15.

Luetcke, H., Gevensleben, H., Albrecht, B., and Frahm, J. (2009). Brain networks involved in early versus late response anticipation and their relation to conflict processing. J Cogn Neurosci 21, 2172-84.

Mathiak, K.A., Koush, Y., Dyck, M., Gaber, T.J., Alawi, E., Zepf, F.D., Zvyagintsev, M., and Mathiak, K. (2010). Social reinforcement can regulate localized brain activity. Eur Arch Psychiatry Clin Neurosci 260 Suppl 2, S132-6.

Menon, V. (2011). Large-scale brain networks and psychopathology: a unifying triple network model. Trends Cogn Sci 15, 483-506.

Menon, V., and Uddin, L. (2010). Saliency, switching, attention and control: a network model of insula function. Brain Struct Funct. 214, 655-67.

Minka, T. (2000). "Automatic choice of dimensionality for PCA", in: Technical Report 514.).

Nee, D., Wager, T., and Jonides, J. (2007). Interference resolution: Insights from a metaanalysis of neuroimaging tasks. Cogn Affect Behav Neurosci. 7, 1-17. 
Niv, S. (2013). Clinical efficacy and potential mechanisms of neurofeedback. Pers Indiv Differ. 54, 676-86.

Peterson, B.S., Kane, M.J., Alexander, G.M., Lacadie, C., Skudlarski, P., Leung, H.-C., May, J., and Gore, J.C. (2002). An event-related functional MRI study comparing interference effects in the Simon and Stroop tasks. Cognitive Brain Research 13, 427-40.

Riccio, C.A., Reynolds, C.R., Lowe, P., and Moore, J.J. (2002). The continuous performance test: a window on the neural substrates for attention? Archives of Clinical Neuropsychology 17, 235-72.

Robineau, F., Rieger, S.W., Mermoud, C., Pichon, S., Koush, Y., Van De Ville, D., Vuilleumier, P., and Scharnowski, F. (2014). Self-regulation of inter-hemispheric visual cortex balance through real-time fMRI neurofeedback training. Neuroimage 100, 1-14.

Ros, T., Théberge, J., Frewen, P.A., Kluetsch, R., Densmore, M., Calhoun, V.D., and Lanius, R.A. (2013). Mind over chatter: Plastic up-regulation of the fMRI salience network directly after EEG neurofeedback. Neuroimage 65, 324-35.

Ruiz, S., Buyukturkoglu, K., Rana, M., Birbaumer, N., and Sitaram, R. (2014). Real-time fMRI brain computer interfaces: Self-regulation of single brain regions to networks. Biological psychology 95, 4-20.

Ruiz, S., Lee, S., Soekadar, S.R., Caria, A., Veit, R., Kircher, T., Birbaumer, N., and Sitaram, R. (2013). Acquired self-control of insula cortex modulates emotion recognition and brain network connectivity in schizophrenia. Hum Brain Mapp 34, 200-12.

Scharnowski, F., Hutton, C., Josephs, O., Weiskopf, N., and Rees, G. (2012). Improving Visual Perception through Neurofeedback. J Neurosci. 32, 17830-41.

Scharnowski, F., Rosa, M.J., Golestani, N., Hutton, C., Josephs, O., Weiskopf, N., and Rees, G. (2014). Connectivity Changes Underlying Neurofeedback Training of Visual Cortex Activity. PLoS One. 9, e91090.

Seeley, W.W., Menon, V., Schatzberg, A.F., Keller, J., Glover, G.H., Kenna, H., Reiss, A.L., and Greicius, M.D. (2007). Dissociable intrinsic connectivity networks for salience processing and executive control. J Neurosci. 27, 2349-56.

Shackman, A.J., Salomons, T.V., Slagter, H.A., Fox, A.S., Winter, J.J., and Davidson, R.J. (2011). The integration of negative affect, pain and cognitive control in the cingulate cortex. Nat Rev Neurosci. 12, 154-67.

Sitaram, R., Veit, R., Stevens, B., Caria, A., Gerloff, C., Birbaumer, N., and Hummel, F. (2012). Acquired control of ventral premotor cortex activity by feedback training: an exploratory real-time FMRI and TMS study. Neurorehabil Neural Repair. 26, 256-65.

Smith, S.M. (2002). Fast robust automated brain extraction. Hum Brain Mapp 17, 143-55.

Sridharan, D., Levitin, D.J., and Menon, V. (2008). A critical role for the right frontoinsular cortex in switching between central-executive and default-mode networks. Proc. Natl. Acad. Sci. U.S.A 105, 12569-74.

Subramanian, L., Hindle, J.V., Johnston, S., Roberts, M.V., Husain, M., Goebel, R., and Linden, D. (2011). Real-Time Functional Magnetic Resonance Imaging Neurofeedback for Treatment of Parkinson's Disease. J Neurosci. 31, 16309-17.

Sulzer, J., Haller, S., Scharnowski, F., Weiskopf, N., Birbaumer, N., Blefari, M.L., Bruehl, A.B., Cohen, L.G., Decharms, R.C., Gassert, R., Goebel, R., Herwig, U., Laconte, S., Linden, D., Luft, A., Seifritz, E., and Sitaram, R. (2013). Real-time fMRI neurofeedback: Progress and challenges. Neuroimage 76, 386-99. 
Tang, Y.Y., Ma, Y.H., Wang, J., Fan, Y.X., Feng, S.G., Lu, Q.L., Yu, Q.B., Sui, D., Rothbart, M.K., Fan, M., and Posner, M.I. (2007). Short-term meditation training improves attention and self-regulation. Proc. Natl. Acad. Sci. U.S.A 104, 17152-6.

Veit, R., Singh, V., Sitaram, R., Caria, A., Rauss, K., and Birbaumer, N. (2012). Using realtime fMRI to learn voluntary regulation of the anterior insula in the presence of threat-related stimuli. Soc Cogn Affect Neurosci. 7, 623-34.

Verbruggen, F., and Logan, G.D. (2008). Response inhibition in the stop-signal paradigm. Trends Cogn Sci 12, 418-24.

Vogt, B.A. (2009). Cingulate Neurobiology and Disease. New York: Oxford University Press.

Weiskopf, N. (2012). Real-time fMRI and its application to neurofeedback. Neuroimage $62,682-92$.

Weiskopf, N., Scharnowski, F., Veit, R., Goebel, R., Birbaumer, N., and Mathiak, K. (2004). Self-regulation of local brain activity using real-time functional magnetic resonance imaging (fMRI). J Physiol-Paris 98, 357-3.

Weiskopf, N., Veit, R., Erb, M., Mathiak, K., Grodd, W., Goebel, R., and Birbaumer, N. (2003). Physiological self-regulation of regional brain activity using real-time functional magnetic resonance imaging (fMRI): methodology and exemplary data. Neuroimage 19, 577-86.

Woolrich, M.W., Ripley, B.D., Brady, M., and Smith, S.M. (2001). Temporal Autocorrelation in Univariate Linear Modeling of FMRI Data. Neuroimage 14, 1370-86.

Worsley, K.J. (2001). "Statistical analysis of activation images," in Functional MRI: An Introduction to Methods, eds. P. Jezzard, P.M. Matthews \& S.M. Smith. Oxford University Press).

Yoo, S.S., Lee, J.H., O'leary, H., Panych, L.P., and Jolesz, F.A. (2008). Neurofeedback fMRImediated learning and consolidation of regional brain activation during motor imagery. Int J Imaging Syst Technol 18, 69-78.

Young, K.D., Zotev, V., Phillips, R., Misaki, M., Yuan, H., Drevets, W.C., and Bodurka, J. (2014). Real-Time fMRI Neurofeedback Training of Amygdala Activity in Patients with Major Depressive Disorder. PLoS One 9, e88785.

Zhang, H., Long, Z.Y., Ge, R.Y., Xu, L.L., Jin, Z., Yao, L., and Liu, Y.J. (2014). Motor Imagery Learning Modulates Functional Connectivity of Multiple Brain Systems in Resting State. PLoS One 9.

Zotev, V., Krueger, F., Phillips, R., Alvarez, R.P., Simmons, W.K., Bellgowan, P., Drevets, W.C., and Bodurka, J. (2011). Self-regulation of amygdala activation using realtime FMRI neurofeedback. PLoS One 6, e24522. 


\section{Abbreviations}

4D

AC-PC

ACC

ADHD

AMCC

ANOVA

BBR

BET

BOLD

CEN

CPT

DMN

EEG

EPI

ETR

FEAT

FILM

FLAME

FLIRT

fMRI

FMRIB

fNIRS

FNIRT

FS

FSL

FTP

FUGUE

FWE

FWHM

GLM

ICA

ICN

IPL

IFG

$\mathrm{M}$

MCFLIRT

MELODIC

Mdn.

MNI

$\mathrm{NF}$

PCC

pgACC

PC

PRELUDE

PSC

PT 4-dimensional

anterior comissure - posterior comissure

anterior cingulate cortex

attention-deficit hyperactivity disorder

anterior mid-cingulate cortex

analysis of variance

boundary-based registration

brain extraction tool

blood oxygen level-dependent

central executive network

continuous performance task

default mode network

electroencephelography

echoplanar imaging

efficient training run

FMRIB's Expert Analysis Tool

FMRIB's Improved Linear Model

FMRIB's Local Analysis of Mixed Effects

fMRIB's Linear Image Registration Tool

functional magnetic resonance imaging

functional magnetic resonance imaging of the brain

functional near-infrared spectroscopy

FMRIB's Nonlinear Image Registration Tool

feedback signal

FMRIB software library

file transfer protocol

FMRIB's Utility for Geometrically Unwarping EPIs

family-wise error

full width at half maximum

general linear model

independent component analysis

intrinsic connectivity network

inferior parietal lobule

inferior frontal gyrus

mean

motion correction FLIRT

multivariate exploratory linear optimized decomposition into

independent components

median

Montreal Neurological Institute

neurofeedback

posterior cingulate cortex

pregenual ACC

Parallel control

Phase Region Expanding Labeller For Unwrapping Discrete Estimates percent signal change

Parallel training 


$\begin{array}{ll}\text { ROI } & \text { region-of-interest } \\ \text { rt-fMRI } & \text { real-time functional magnetic resonance imaging } \\ \text { SAM } & \text { self-assessement manikins } \\ \text { SC } & \text { Serial control } \\ \text { SCP } & \text { slow cortical potentials } \\ \text { SD } & \text { standard deviation } \\ \text { SEM } & \text { standard error of the mean } \\ \text { SgACC } & \text { subgenual ACC } \\ \text { SMA } & \text { supplementary motor area } \\ \text { SMC } & \text { somatomotor cortex } \\ \text { SN } & \text { salience network } \\ \text { SPM } & \text { Statistical Parametric Mapping } \\ \text { SPSS } & \text { Statistical Package for the Social Sciences } \\ \text { ST } & \text { Serial training } \\ \text { TBV } & \text { Turbo Brain Voyager } \\ \text { T1 } & \text { Longitudinal relaxation time } \\ \text { TE } & \text { echo time } \\ \text { TR } & \text { repetition time } \\ \text { vmPFC } & \text { ventromedial pre-frontal cortex } \\ \text { vS. } & \text { versus }\end{array}$




\section{Appendix}

\subsection{Questionnaires}

\subsubsection{Example of a pre-session questionnaire}

(handed out in the pre-training transfer sesion)

Volunteer number:

Volunter initials:

Date:

Dear participants,

Thank you for your time and interest in taking part of this neurofeedback experiment.

Before you begin, we would like to know more about your mood for today.

Please mark with an $\mathbf{X}$ in the appropriate cartoon figure, one box per question:

1. Rate your current mood

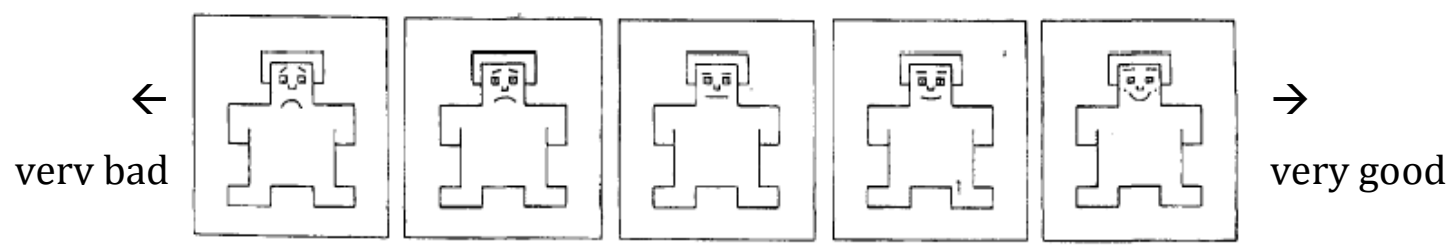

2. Rate your current energy level

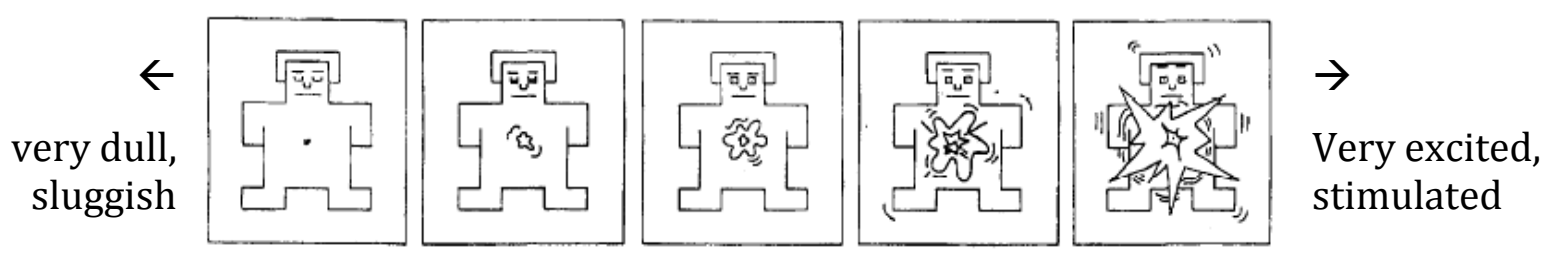

3. Do you have any experience in meditation or any practice to control breathing? Please state the type of meditation, whether you practice it regularly, and how long have you been practicing it. 
Appendix

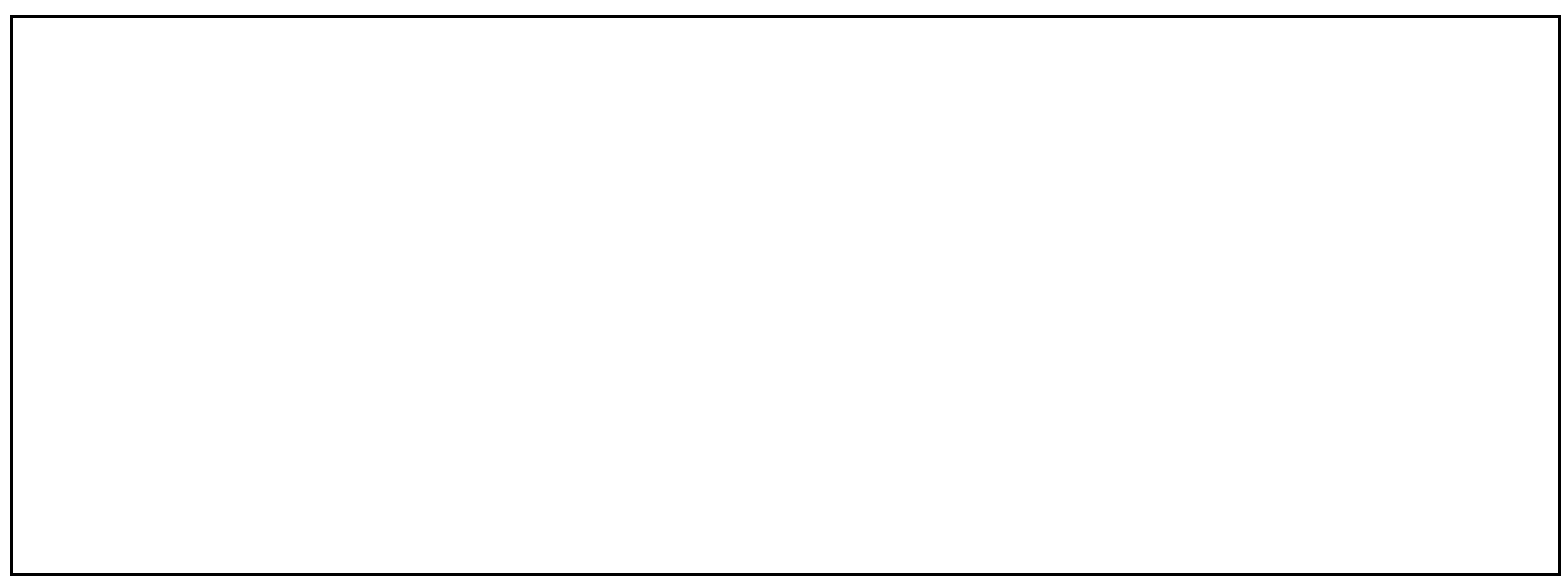

Thank you! 


\subsubsection{Example of a post-session questionnaire for Serial paradigm}

Volunteer number:

Initials:

Date:

Dear participants,

Thank you for your time and interest in taking part of this neurofeedback experiment. Before you leave, we have just one more thing for you to do now. We would like to know what was going through your mind during the experiment. There is no right or wrong answer, just please answer as honestly as possible.

Data that you provide in this survey will be anonymous.

Please mark with an $\mathbf{X}$ / click (computer version) in the appropriate box, one box per question:

4. How did you find the task during the

think phase?

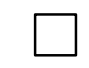

$\square$

Difficult Very difficult
Neither
easy nor

difficult

5. What do you think about your performance during the think phase?
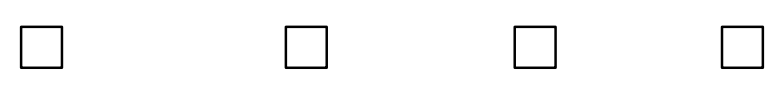

Easy Very easy

r


8. Did you somehow fall asleep during the training?

$\begin{array}{ccc}\text { No } & \text { Don't } & \text { Yes } \\ \text { know / } & \\ \text { Not sure } & \end{array}$

9. How dominant (in control) did you feel during the neurofeedback training task?

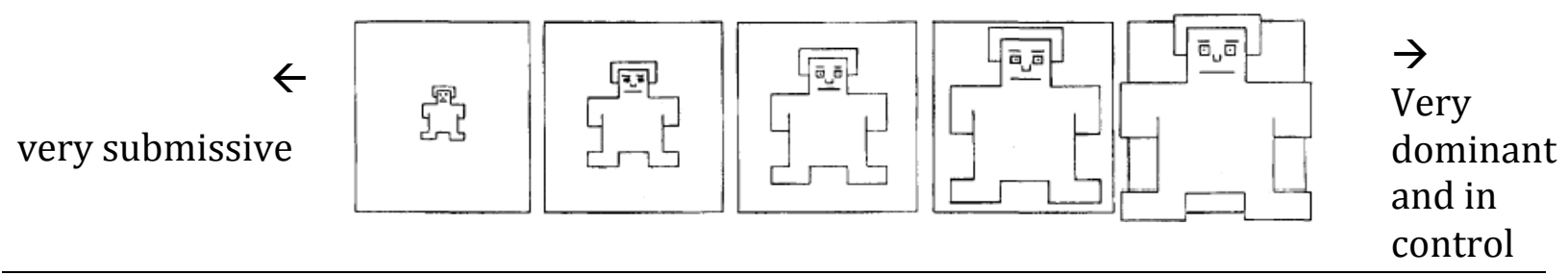

10. Rate your current

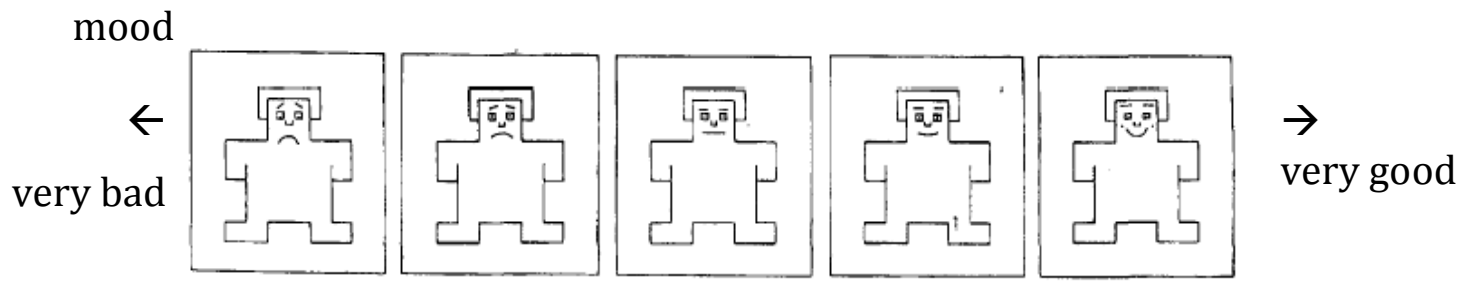

11. Rate your current energy level

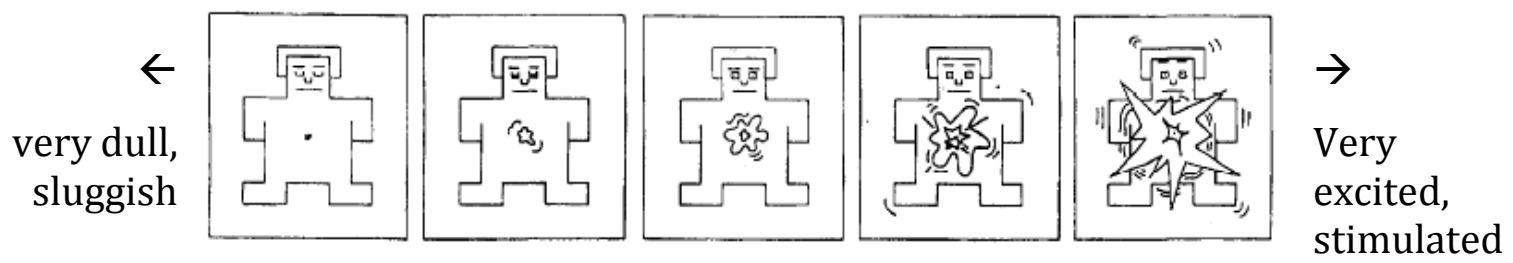

See next page.. 
12. What was your mental strategy during the "think" phase of the neurofeedback training?

Please describe as many as you can remember, and as detailed as possible.

13. Was something going through your mind during the "feedback" of think phase?

14. What was your mental strategy during the "count" phase of the neurofeedback training?

Please describe if you did something other than counting. 
Appendix

15. Was something going through your mind during the "feedback" of count phase

\section{Thank you!}




\subsubsection{Example of a post-session questionnaire for Parallel paradigm}
Volunteer number:
Initials:
Date:

Dear participants,

Thank you for your time and interest in taking part of this neurofeedback experiment. Before you leave, we have just one more thing for you to do now. We would like to know what was going through your mind during the experiment. There is no right or wrong answer, just please answer as honestly as possible.

Data that you provide in this survey will be anonymous.

Please mark with an $\mathbf{X}$ / click (computer version) in the appropriate box, one box per question:

1. How did you find the task during the

think phase?

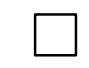

$\square$

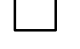

Difficult difficult
Neither easy nor difficult

2. What do you think about your performance during the think phase?

difficult

\section{Easy Very easy}
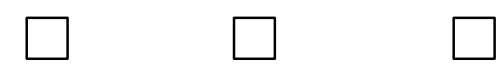
5. Did you somehow

fall asleep during

the training?

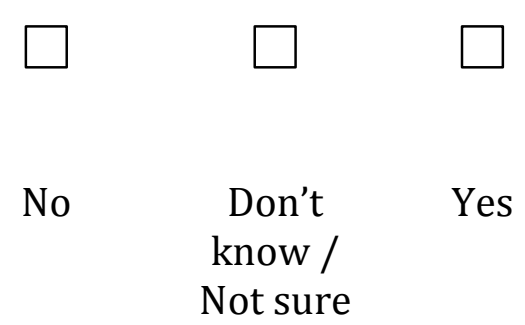

know /

Not sure

6. How dominant (in control) did you feel during the neurofeedback training task?

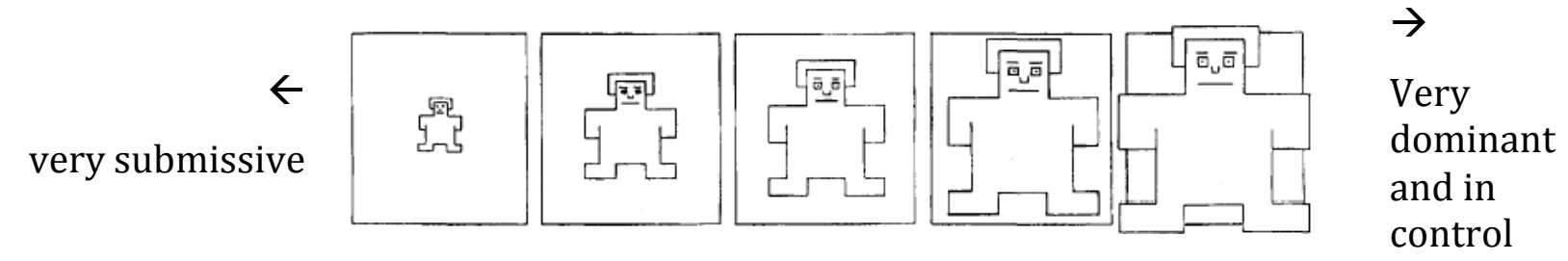

7. Rate your current

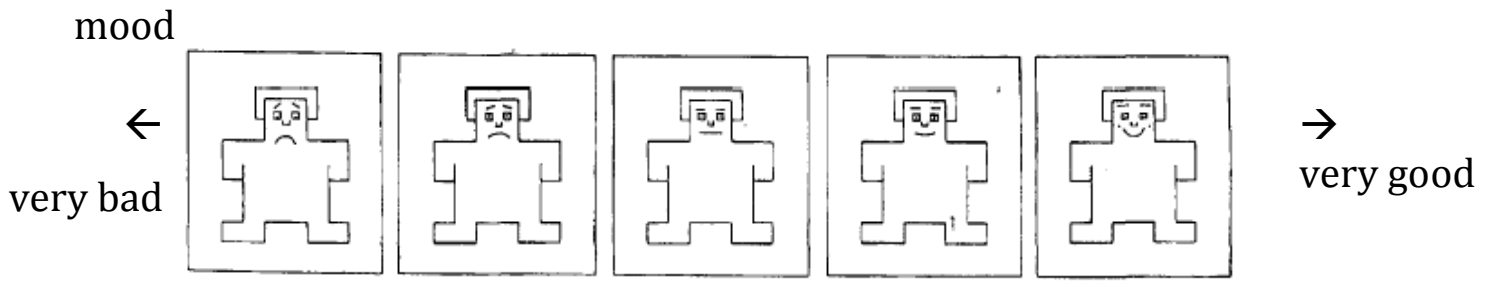

8. Rate your current energy

level

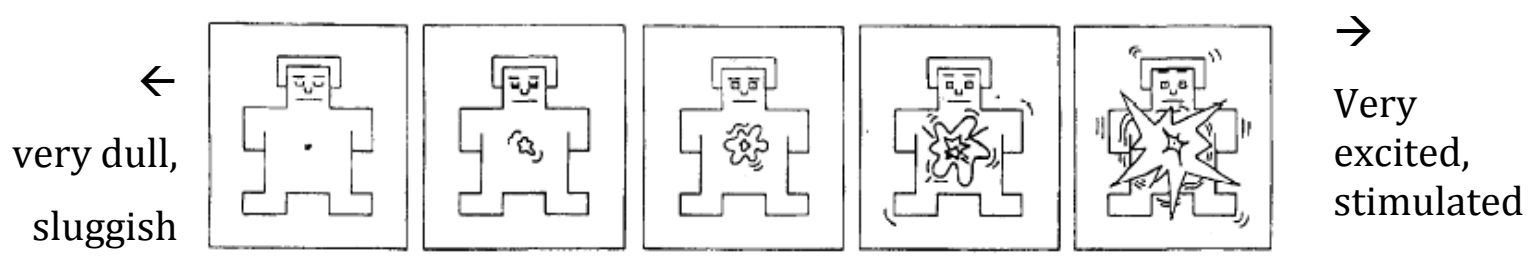


9. What was your mental strategy during the "think" phase of the neurofeedback training?

Please describe as many as you can remember, and as detailed as possible.

10. What was your mental strategy during the "count" phase of the neurofeedback training?

Please describe if you did something other than counting.

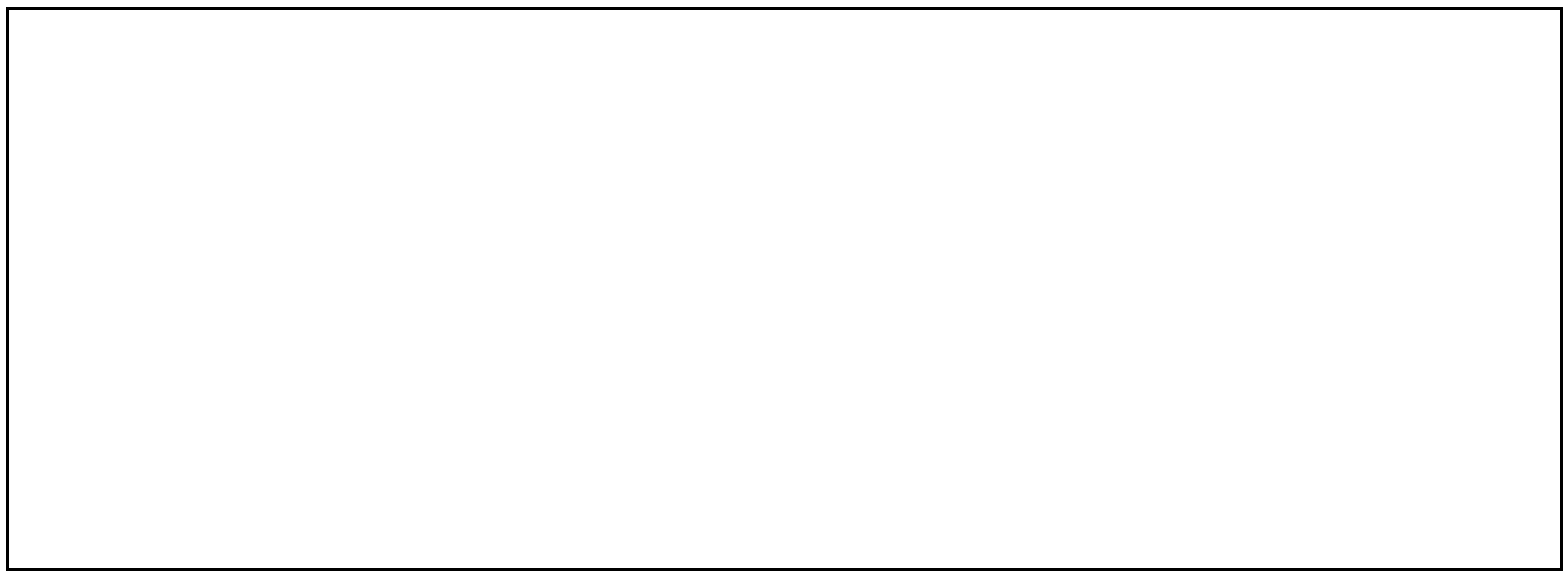

Thank you! 


\subsection{Outputs of statistical tests from SPSS}

\subsubsection{Rt-fMRI cognitive neurofeedback training of the aMCC}

\subsubsection{3-way ANOVA for Transfer Condition (PARADIGM, TRAINING GROUP, TIME)}

Within-Subjects

Factors

Between-Subjects Factors

\begin{tabular}{|l|l|} 
Measure: MEASURE_1 \\
time & $\begin{array}{c}\text { Dependent } \\
\text { Variable }\end{array}$ \\
\hline 1 & NFB_pre \\
2 & NFB_post \\
\hline
\end{tabular}

\begin{tabular}{|ll|l|r|}
\hline & & Value Label & N \\
\hline Paradigm & 1.00 & Serial & 15 \\
& 2.00 & Parallel & 15 \\
Group & 0 & Controls & 10 \\
& 1 & Train & 20 \\
\hline
\end{tabular}

Tests of Within-Subjects Contrasts

Measure: MEASURE_1

\begin{tabular}{|ll|r|r|r|r|r|}
\hline Source & time & \multicolumn{1}{c|}{$\begin{array}{c}\text { Type III Sum } \\
\text { of Squares }\end{array}$} & \multicolumn{1}{c|}{ df } & Mean Square & \multicolumn{1}{c|}{ F } & \multicolumn{1}{c|}{ Sig. } \\
\hline time & Level 1 vs. Level 2 & 2.429 & 1 & 2.429 & .877 & .358 \\
\hline time * Paradigm & Level 1 vs. Level 2 & .088 & 1 & .088 & .032 & .860 \\
\hline time * Training & Level 1 vs. Level 2 & 3.280 & 1 & 3.280 & 1.184 & .287 \\
\hline $\begin{array}{l}\text { time * Paradigm * } \\
\text { Training }\end{array}$ & Level 1 vs. Level 2 & .906 & 1 & .906 & .327 & .572 \\
\hline Error(time) & Level 1 vs. Level 2 & 72.037 & 26 & 2.771 & & \\
\hline
\end{tabular}

\section{Tests of Between-Subjects Effects}

Measure: MEASURE_1

Transformed Variable: Average

\begin{tabular}{|l|r|r|r|r|r|}
\hline Source & $\begin{array}{c}\text { Type III Sum } \\
\text { of Squares }\end{array}$ & df & Mean Square & \multicolumn{1}{c|}{ F } & Sig. \\
\hline Intercept & 10.965 & 1 & 10.965 & 24.840 & .000 \\
Paradigm & 3.548 & 1 & 3.548 & 8.036 & .009 \\
Training & .014 & 1 & .014 & .031 & .861 \\
Paradigm * Training & .150 & 1 & .150 & .340 & .565 \\
Error & 11.478 & 26 & .441 & & \\
\hline
\end{tabular}
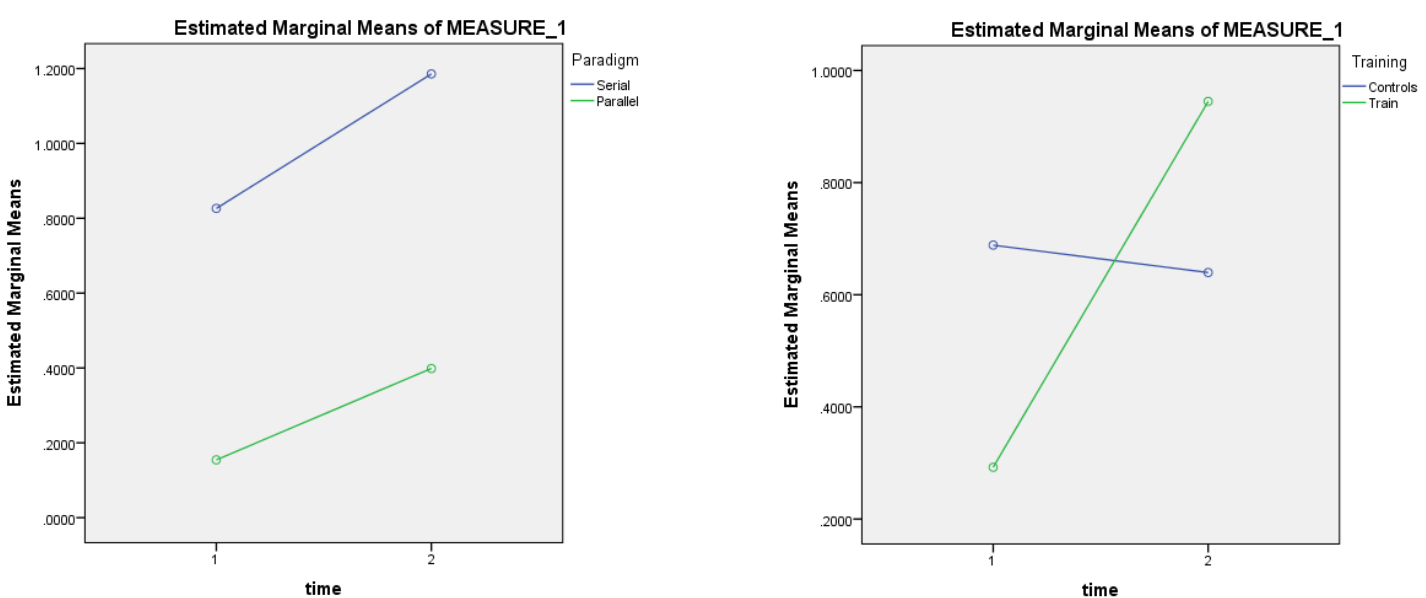
9.2.1.2 2-way ANOVA for Transfer Condition (LEARNING STATUS, TIME)

\begin{tabular}{|c|c|c|c|c|c|}
\hline \multirow{2}{*}{\multicolumn{4}{|c|}{ Between-Subjects Factors }} & \multicolumn{2}{|c|}{$\begin{array}{l}\text { Within-Subjects } \\
\text { Factors }\end{array}$} \\
\hline & & & & \multicolumn{2}{|c|}{ Measure: MEASURE_ } \\
\hline & & Value Label & $N$ & \multirow[b]{2}{*}{ time } & \multirow{2}{*}{$\begin{array}{c}\text { Dependent } \\
\text { Variable }\end{array}$} \\
\hline \multirow[t]{2}{*}{ Learner } & .00 & NonLearner & 12 & & \\
\hline & 1.00 & Learner & 8 & 2 & NFB_post \\
\hline
\end{tabular}

Tests of Within-Subjects Contrasts

Measure: MEASURE_1

\begin{tabular}{|ll|r|r|r|r|r|}
\hline Source & time & $\begin{array}{c}\text { Type III Sum } \\
\text { of Squares }\end{array}$ & \multicolumn{1}{c|}{ df } & Mean Square & \multicolumn{1}{c|}{ F } & \multicolumn{1}{c|}{ Sig. } \\
\hline time & Level 2 vs. Level 1 & 15.877 & 1 & 15.877 & 7.938 & .011 \\
\hline time * Learner & Level 2 vs. Level 1 & 31.660 & 1 & 31.660 & 15.828 & .001 \\
\hline Error(time) & Level 2 vs. Level 1 & 36.003 & 18 & 2.000 & & \\
\hline
\end{tabular}

Tests of Between-Subjects Effects

Measure: MEASURE_1

Transformed Variable: Average

\begin{tabular}{|l|r|r|r|r|r|}
\hline Source & $\begin{array}{c}\text { Type III Sum } \\
\text { of Squares }\end{array}$ & df & Mean Square & \multicolumn{1}{c|}{ F } & Sig. \\
\hline Intercept & 8.794 & 1 & 8.794 & 15.522 & .001 \\
Learner & 1.628 & 1 & 1.628 & 2.874 & .107 \\
Error & 10.198 & 18 & .567 & & \\
\hline
\end{tabular}




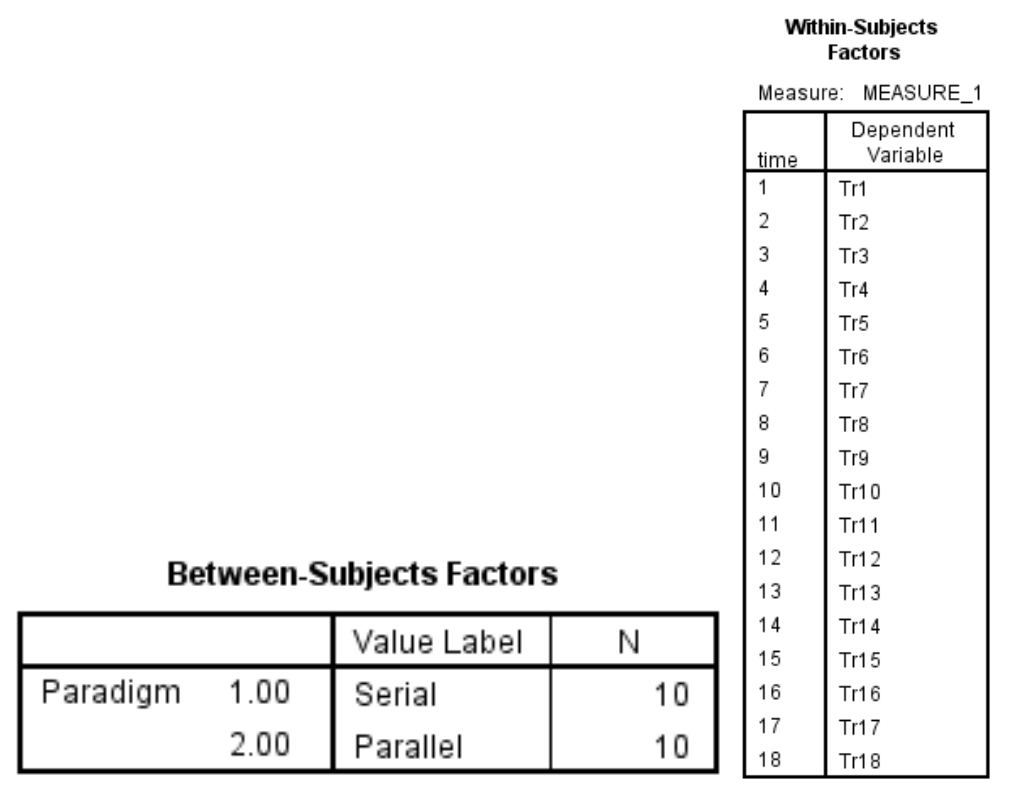

Tests of Within-Subjects Effects

Measure: MEASURE_1

\begin{tabular}{|ll|r|r|r|r|r|}
\hline Source & \multicolumn{1}{c|}{$\begin{array}{c}\text { Type III Sum } \\
\text { of Squares }\end{array}$} & \multicolumn{1}{c|}{ df } & Mean Square & F & Sig. \\
\hline time & Sphericity Assumed & 7.259 & 17 & .427 & .901 & .573 \\
& Greenhouse-Geisser & 7.259 & 4.624 & 1.570 & .901 & .478 \\
& Huynh-Feldt & 7.259 & 6.764 & 1.073 & .901 & .506 \\
& Lower-bound & 7.259 & 1.000 & 7.259 & .901 & .355 \\
\hline time * Paradigm & Sphericity Assumed & 6.315 & 17 & .371 & .784 & .712 \\
& Greenhouse-Geisser & 6.315 & 4.624 & 1.366 & .784 & .555 \\
& Huynh-Feldt & 6.315 & 6.764 & .934 & .784 & .598 \\
& Lower-bound & 6.315 & 1.000 & 6.315 & .784 & .388 \\
\hline Error(time) & Sphericity Assumed & 145.005 & 306 & .474 & & \\
& Greenhouse-Geisser & 145.005 & 83.229 & 1.742 & & \\
& Huynh-Feldt & 145.005 & 121.753 & 1.191 & & \\
& Lower-bound & 145.005 & 18.000 & 8.056 & & \\
\hline
\end{tabular}

\section{Tests of Between-Subjects Effects}

Measure: MEASURE_1

Transformed Variable: Average

\begin{tabular}{|l|r|r|r|r|r|}
\hline Source & \multicolumn{1}{|c|}{$\begin{array}{c}\text { Type III Sum } \\
\text { of Squares }\end{array}$} & df & Mean Square & \multicolumn{1}{c|}{ F } & \multicolumn{1}{c|}{ Sig. } \\
\hline Intercept & 6.364 & 1 & 6.364 & 17.713 & .001 \\
Paradigm & 3.319 & 1 & 3.319 & 9.237 & .007 \\
Error & 6.467 & 18 & .359 & & \\
\hline
\end{tabular}


9.2.1.4 2-way ANOVA for Training Condition (LEARNING STATUS, TIME) Within-Subjects Factors

Measure: MEASURE_1
\begin{tabular}{|l|l|}
\hline time & $\begin{array}{c}\text { Dependent } \\
\text { Variable }\end{array}$ \\
\hline 1 & $\operatorname{Tr} 1$ \\
2 & $\operatorname{Tr} 2$ \\
3 & $\operatorname{Tr} 3$ \\
4 & $\operatorname{Tr} 4$ \\
5 & $\operatorname{Tr} 5$ \\
6 & $\operatorname{Tr} 6$ \\
7 & $\operatorname{Tr} 7$ \\
8 & $\operatorname{Tr} 8$ \\
9 & $\operatorname{Tr} 9$ \\
10 & $\operatorname{Tr} 10$ \\
11 & $\operatorname{Tr} 11$ \\
12 & $\operatorname{Tr} 12$ \\
13 & $\operatorname{Tr} 13$ \\
14 & $\operatorname{Tr} 14$ \\
15 & $\operatorname{Tr} 15$ \\
16 & $\operatorname{Tr} 16$ \\
17 & $\operatorname{Tr} 17$ \\
18 & $\operatorname{Tr} 18$ \\
\hline
\end{tabular}

$$
\sqrt{1}
$$

Between-Subjects Factors

\begin{tabular}{|rl|l|r|}
\hline & & Value Label & \multicolumn{1}{|c|}{$\mathrm{N}$} \\
\hline Learner & .00 & NonLearner & 12 \\
& 1.00 & Learner & 8 \\
\hline
\end{tabular}

Tests of Within-Subjects Effects

Measure: MEASURE_1

\begin{tabular}{|c|c|c|c|c|c|c|}
\hline Source & & $\begin{array}{c}\text { Type III Sum } \\
\text { of Squares }\end{array}$ & df & Mean Square & $\mathrm{F}$ & Sig. \\
\hline \multirow[t]{4}{*}{ time } & Sphericity Assumed & 9.376 & 17 & .552 & 1.237 & .234 \\
\hline & Greenhouse-Geisser & 9.376 & 5.291 & 1.772 & 1.237 & .297 \\
\hline & Huynh-Feldt & 9.376 & 8.170 & 1.148 & 1.237 & .280 \\
\hline & Lower-bound & 9.376 & 1.000 & 9.376 & 1.237 & .281 \\
\hline \multirow[t]{4}{*}{ time * Learner } & Sphericity Assumed & 14.935 & 17 & .879 & 1.971 & .013 \\
\hline & Greenhouse-Geisser & 14.935 & 5.291 & 2.823 & 1.971 & .086 \\
\hline & Huynh-Feldt & 14.935 & 8.170 & 1.828 & 1.971 & .053 \\
\hline & Lower-bound & 14.935 & 1.000 & 14.935 & 1.971 & .177 \\
\hline \multirow[t]{4}{*}{ Error(time) } & Sphericity Assumed & 136.384 & 306 & .446 & & \\
\hline & Greenhouse-Geisser & 136.384 & 95.245 & 1.432 & & \\
\hline & Huynh-Feldt & 136.384 & 147.058 & .927 & & \\
\hline & Lower-bound & 136.384 & 18.000 & 7.577 & & \\
\hline
\end{tabular}

Tests of Between-Subjects Effects

Measure: MEASURE_1

Transformed Variable: Average

\begin{tabular}{|l|r|r|r|r|r|}
\hline Source & $\begin{array}{c}\text { Type III Sum } \\
\text { of Squares }\end{array}$ & \multicolumn{1}{c|}{ df } & Mean Square & \multicolumn{1}{c|}{ F } & \multicolumn{1}{c|}{ Sig. } \\
\hline Intercept & 7.733 & 1 & 7.733 & 18.815 & .000 \\
Learner & 2.388 & 1 & 2.388 & 5.810 & .027 \\
Error & 7.398 & 18 & .411 & & \\
\hline
\end{tabular}


Appendix

9.2.1.5 2-way ANOVA for Feedback appraisal (TASK, TIME)

Within-Subjects Factors

Measure: MEASURE_1

\begin{tabular}{|ll|l|}
\hline & & $\begin{array}{c}\text { Dependent } \\
\text { Variable }\end{array}$ \\
\hline 1 & 1 & Tr1 \\
& 2 & FB1 \\
\hline 2 & 1 & Tr2 \\
& 2 & FB2 \\
\hline 3 & 1 & Tr3 \\
& 2 & FB3 \\
\hline 4 & 1 & Tr4 \\
& 2 & FB4 \\
\hline 5 & 1 & Tr5 \\
& 2 & FB5 \\
\hline 6 & 1 & Tr6 \\
& 2 & FB6 \\
\hline 7 & 1 & Tr7 \\
& 2 & FB7 \\
\hline 8 & 1 & Tr8 \\
& 2 & FB8 \\
\hline 9 & 1 & Tr9 \\
& 2 & FB9 \\
\hline 10 & 1 & Tr10 \\
& 2 & FB10 \\
\hline 11 & 1 & Tr11 \\
& 2 & FB11 \\
\hline 12 & 1 & Tr12 \\
& 2 & FB12 \\
\hline 13 & 1 & Tr13 \\
& 2 & FB13 \\
\hline 14 & 1 & Tr14 \\
& 2 & FB14 \\
\hline 15 & 1 & Tr15 \\
& 2 & FB15 \\
\hline 16 & 1 & Tr16 \\
& 2 & FB16 \\
\hline 17 & 1 & Tr17 \\
& 2 & FB17 \\
\hline
\end{tabular}

Tests of Within-Subjects Effects

Measure: MEASURE_1

\begin{tabular}{|c|c|c|c|c|c|c|c|}
\hline Training & Source & & $\begin{array}{l}\text { Type III Sum } \\
\text { of Squares }\end{array}$ & df & Mean Square & $\mathrm{F}$ & Sig. \\
\hline \multirow[t]{24}{*}{ Train } & \multirow[t]{4}{*}{ time } & Sphericity Assumed & 4.221 & 17 & .248 & 1.008 & .452 \\
\hline & & Greenhouse-Geisser & 4.221 & 5.153 & .819 & 1.008 & .425 \\
\hline & & Huynh-Feldt & 4.221 & 12.878 & .328 & 1.008 & .448 \\
\hline & & Lower-bound & 4.221 & 1.000 & 4.221 & 1.008 & .342 \\
\hline & \multirow[t]{4}{*}{ Error(time) } & Sphericity Assumed & 37.671 & 153 & .246 & & \\
\hline & & Greenhouse-Geisser & 37.671 & 46.381 & .812 & & \\
\hline & & Huynh-Feldt & 37.671 & 115.900 & .325 & & \\
\hline & & Lower-bound & 37.671 & 9.000 & 4.186 & & \\
\hline & \multirow[t]{4}{*}{ task } & Sphericity Assumed & 74.864 & 1 & 74.864 & 16.929 & .003 \\
\hline & & Greenhouse-Geisser & 74.864 & 1.000 & 74.864 & 16.929 & .003 \\
\hline & & Huynh-Feldt & 74.864 & 1.000 & 74.864 & 16.929 & .003 \\
\hline & & Lower-bound & 74.864 & 1.000 & 74.864 & 16.929 & .003 \\
\hline & \multirow[t]{4}{*}{ Error(task) } & Sphericity Assumed & 39.801 & 9 & 4.422 & & \\
\hline & & Greenhouse-Geisser & 39.801 & 9.000 & 4.422 & & \\
\hline & & Huynh-Feldt & 39.801 & 9.000 & 4.422 & & \\
\hline & & Lower-bound & 39.801 & 9.000 & 4.422 & & \\
\hline & \multirow[t]{4}{*}{ time * task } & Sphericity Assumed & 4.328 & 17 & .255 & .755 & .742 \\
\hline & & Greenhouse-Geisser & 4.328 & 3.633 & 1.191 & .755 & .551 \\
\hline & & Huynh-Feldt & 4.328 & 6.395 & .677 & .755 & .616 \\
\hline & & Lower-bound & 4.328 & 1.000 & 4.328 & .755 & .408 \\
\hline & \multirow[t]{4}{*}{ Error(time*task) } & Sphericity Assumed & 51.597 & 153 & .337 & & \\
\hline & & Greenhouse-Geisser & 51.597 & 32.693 & 1.578 & & \\
\hline & & Huynh-Feldt & 51.597 & 57.555 & .896 & & \\
\hline & & Lower-bound & 51.597 & 9.000 & 5.733 & & \\
\hline
\end{tabular}




\subsubsection{Cross-tabulation (PARADIGM, LEARNING STATUS)}

Paradigm * Learner Crosstabulation

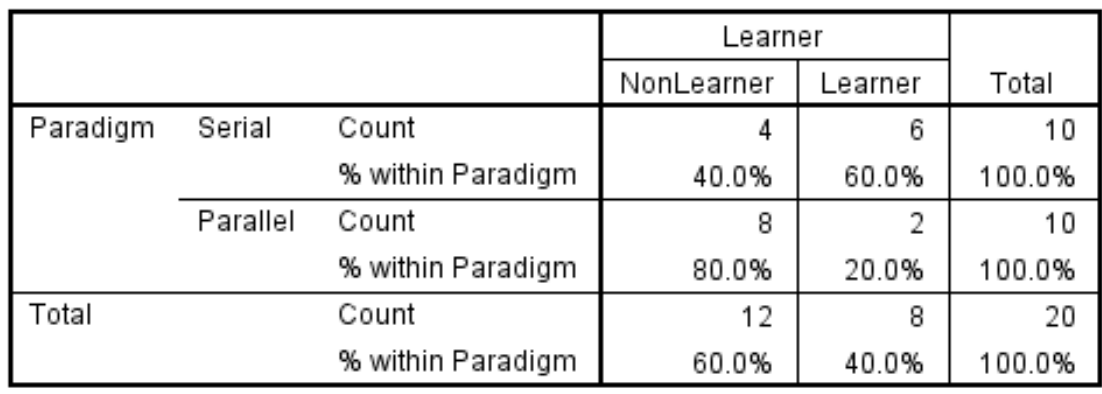

Chi-Square Tests

\begin{tabular}{|l|r|r|r|r|r|}
\hline & Value & df & $\begin{array}{c}\text { Asymp. Sig. } \\
\text { (2-sided) }\end{array}$ & $\begin{array}{c}\text { Exact Sig. (2- } \\
\text { sided) }\end{array}$ & $\begin{array}{c}\text { Exact Sig. (1- } \\
\text { sided) }\end{array}$ \\
\hline Pearson Chi-Square & $3.333^{\mathrm{a}}$ & 1 & .068 & .170 & .085 \\
Continuity Correction $^{\mathrm{b}}$ & 1.875 & 1 & .171 & & \\
Likelihood Ratio & 3.452 & 1 & .063 & .170 & .085 \\
Fisher's Exact Test & & & & .170 & .085 \\
N of Valid Cases & 20 & & & & \\
\hline
\end{tabular}

a. 2 cells (50.0\%) have expected count less than 5 . The minimum expected count is 4.00 .

b. Computed only for a $2 \times 2$ table

\subsubsection{T-test (ETR in LEARNING STATUS)}

\section{Group Statistics}

\begin{tabular}{|ll|r|r|r|r|}
\hline & & \multicolumn{1}{c|}{ Std. } & \multicolumn{1}{c|}{$\begin{array}{c}\text { Std. Error } \\
\text { Mean }\end{array}$} \\
\hline ETR & learn status & $\mathrm{N}$ & Mean & \multicolumn{1}{c|}{ Deviation } & \multicolumn{1}{c|}{ non-learner } \\
& learner & 8 & 6.00 & 4.843 & 1.398 \\
& 8 & 13.00 & 6.459 & 2.283 \\
\hline
\end{tabular}

\begin{tabular}{|c|c|c|c|c|c|c|c|c|c|c|}
\hline & & \multicolumn{2}{|c|}{$\begin{array}{l}\text { Levene's Test for Equality of } \\
\text { Variances }\end{array}$} & \multicolumn{7}{|c|}{ t-test for Equality of Means } \\
\hline & & \multirow[b]{2}{*}{$\mathrm{F}$} & \multirow[b]{2}{*}{ Sig. } & \multirow[b]{2}{*}{$\mathrm{t}$} & \multirow[b]{2}{*}{$\mathrm{df}$} & \multirow{2}{*}{$\begin{array}{l}\text { Sig. (2- } \\
\text { tailed) }\end{array}$} & \multirow{2}{*}{$\begin{array}{c}\text { Mean } \\
\text { Difference }\end{array}$} & \multirow{2}{*}{$\begin{array}{l}\text { Std. Error } \\
\text { Difference }\end{array}$} & \multicolumn{2}{|c|}{$\begin{array}{l}95 \% \text { Confidence Interval of } \\
\text { the Difference }\end{array}$} \\
\hline & & & & & & & & & Lower & Upper \\
\hline ETR & $\begin{array}{l}\text { Equal variances } \\
\text { assumed }\end{array}$ & 1.108 & .307 & -2.774 & 18 & .013 & -7.000 & 2.523 & -12.301 & -1.699 \\
\hline & $\begin{array}{l}\text { Equal variances not } \\
\text { assumed }\end{array}$ & & & -2.614 & 12.145 & .022 & -7.000 & 2.677 & -12.826 & -1.174 \\
\hline
\end{tabular}




\subsubsection{Wilcoxon Signed Rank Test}

\subsection{Self-report on perception of difficulty and performance}

Test Statistics ${ }^{\mathrm{a}}$

\begin{tabular}{|c|c|c|c|c|c|}
\hline \multicolumn{2}{|c|}{ Neurofeedback paradiam } & $\begin{array}{l}\text { Post Self- } \\
\text { perception of } \\
\text { difficulty of } \\
\text { think phase } \\
\text { (reversed) - } \\
\text { Pre Self- } \\
\text { perception of } \\
\text { difficulty of } \\
\text { think phase }\end{array}$ & $\begin{array}{l}\text { Post Self- } \\
\text { perception of } \\
\text { performance } \\
\text { during think } \\
\text { phase - Pre } \\
\text { Self- } \\
\text { perception of } \\
\text { performance } \\
\text { during think } \\
\text { phase }\end{array}$ & $\begin{array}{l}\text { Post Self- } \\
\text { perception of } \\
\text { difficulty } \\
\text { "count' phase } \\
\text { (reversed) - } \\
\text { Pre Self- } \\
\text { perception of } \\
\text { difficulty } \\
\text { "count" phase }\end{array}$ & $\begin{array}{l}\text { Post Self- } \\
\text { perception of } \\
\text { performance } \\
\text { during count } \\
\text { phase - Pre } \\
\text { Self- } \\
\text { perception of } \\
\text { performance } \\
\text { during count } \\
\text { phase }\end{array}$ \\
\hline \multirow{5}{*}{ Serial } & z & $-2.640^{\mathrm{b}}$ & $-2.121^{c}$ & $-2.333^{\circ}$ & $-1.342^{b}$ \\
\hline & Asymp. Sig. (2-tailed) & .008 & .034 & .020 & .180 \\
\hline & Exact Sig. (2-tailed) & .008 & .070 & .031 & .375 \\
\hline & Exact Sig. (1-tailed) & .004 & .035 & .016 & .188 \\
\hline & Point Probability & .004 & .031 & .016 & .156 \\
\hline \multirow{5}{*}{ Parallel } & $z$ & $-.264^{b}$ & $-.577^{\mathrm{b}}$ & $-.816^{\circ}$ & $-.707^{b}$ \\
\hline & Asymp. Sig. (2-tailed) & .792 & .564 & .414 & .480 \\
\hline & Exact Sig. (2-tailed) & .984 & 1.000 & .688 & .750 \\
\hline & Exact Sig. (1-tailed) & .492 & .500 & .344 & .375 \\
\hline & Point Probability & .156 & .375 & .234 & .219 \\
\hline
\end{tabular}
a. Wilcoxon Signed Ranks Test
b. Based on positive ranks.
c. Based on negative ranks. 
Test Statistics ${ }^{\text {a }}$

\begin{tabular}{|c|c|c|c|c|}
\hline \multicolumn{2}{|c|}{ Neurofeedback paradigm } & $\begin{array}{l}\text { Self-reported } \\
\text { dominance } \\
\text { during Post- } \\
\text { training - Self- } \\
\text { reported } \\
\text { dominance } \\
\text { during Pre- } \\
\text { training } \\
\end{array}$ & $\begin{array}{l}\text { Self-reported } \\
\text { Mood/valence } \\
\text { during Post- } \\
\text { training - Self- } \\
\text { reported } \\
\text { Mood/valence } \\
\text { during Pre- } \\
\text { training }\end{array}$ & $\begin{array}{l}\text { Self-reported } \\
\text { arousal } \\
\text { during Post- } \\
\text { training - Self- } \\
\text { reported } \\
\text { arousal } \\
\text { during Pre- } \\
\text { training }\end{array}$ \\
\hline \multirow{5}{*}{ Serial } & $\mathrm{Z}$ & $-2.449^{b}$ & $-1.000^{c}$ & $.000^{\mathrm{d}}$ \\
\hline & Asymp. Sig. (2-tailed) & .014 & .317 & 1.000 \\
\hline & Exact Sig. (2-tailed) & .031 & .625 & 1.000 \\
\hline & Exact Sig. (1-tailed) & .016 & .313 & .656 \\
\hline & Point Probability & .016 & .250 & .313 \\
\hline \multirow[t]{5}{*}{ Parallel } & $\mathrm{Z}$ & $-.378^{b}$ & $-1.134^{b}$ & $.000^{\mathrm{d}}$ \\
\hline & Asymp. Sig. (2-tailed) & .705 & .257 & 1.000 \\
\hline & Exact Sig. (2-tailed) & 1.000 & .500 & 1.000 \\
\hline & Exact Sig. (1-tailed) & .500 & .250 & .625 \\
\hline & Point Probability & .250 & .188 & .250 \\
\hline
\end{tabular}
a. Wilcoxon Signed Ranks Test
b. Based on negative ranks.
c. Based on positive ranks.
d. The sum of negative ranks equals the sum of positive ranks. 


\subsubsection{Generalization of neurofeedback training to behavior}

\subsubsection{3-way ANOVA for CPT (PARADIGM, GROUP, TIME)}

\subsection{Reaction time}

Within-Subjects

Factors

\begin{tabular}{|c|c|c|c|c|c|}
\hline \multicolumn{2}{|c|}{ Measure: MEASURE_1 } & \multicolumn{4}{|c|}{ Between-Subjects Factors } \\
\hline \multirow[b]{2}{*}{ time } & \multirow{2}{*}{$\begin{array}{c}\text { Dependent } \\
\text { Variable }\end{array}$} & & & Value Label & $\mathrm{N}$ \\
\hline & & \multirow[t]{2}{*}{ Paradigm } & & \multirow{4}{*}{$\begin{array}{l}\text { Serial } \\
\text { Parallel } \\
\text { Controls } \\
\text { Train }\end{array}$} & \multirow{2}{*}{$\begin{array}{l}15 \\
15\end{array}$} \\
\hline 1 & CPT it pre & & 2.00 & & \\
\hline & & Training & 0 & & 10 \\
\hline 2 & CPT_rt_post & & 1 & & 20 \\
\hline
\end{tabular}

\section{Tests of Within-Subjects Contrasts}

Measure: MEASURE_1

\begin{tabular}{|ll|r|r|r|r|r|}
\hline Source & time & \multicolumn{1}{c|}{$\begin{array}{c}\text { Type III Sum } \\
\text { of Squares }\end{array}$} & \multicolumn{1}{c|}{ df } & Mean Square & \multicolumn{1}{c|}{ F } & Sig. \\
\hline time & Level 1 vs. Level 2 & 43309.067 & 1 & 43309.067 & 5.903 & .022 \\
\hline time * Paradigm & Level 1 vs. Level 2 & 8640.000 & 1 & 8640.000 & 1.178 & .288 \\
\hline time * Training & Level 1 vs. Level 2 & 1306.667 & 1 & 1306.667 & .178 & .676 \\
\hline $\begin{array}{l}\text { time * Paradigm * } \\
\text { Training }\end{array}$ & Level 1 vs. Level 2 & 1058.400 & 1 & 1058.400 & .144 & .707 \\
\hline Error(time) & Level 1 vs. Level 2 & 190757.600 & 26 & 7336.831 & & \\
\hline
\end{tabular}

Tests of Between-Subjects Effects

Measure: MEASURE_1

Transformed Variable: Average

\begin{tabular}{|l|r|r|r|r|r|}
\hline Source & \multicolumn{1}{|c|}{$\begin{array}{c}\text { Type III Sum } \\
\text { of Squares }\end{array}$} & df & Mean Square & \multicolumn{1}{c|}{ F } & \multicolumn{1}{c|}{ Sig. } \\
\hline Intercept & 6592209.067 & 1 & 6592209.067 & 438.785 & .000 \\
Paradigm & 6869.400 & 1 & 6869.400 & .457 & .505 \\
Training & 601.667 & 1 & 601.667 & .040 & .843 \\
Paradigm * Training & 1706.667 & 1 & 1706.667 & .114 & .739 \\
Error & 390618.600 & 26 & 15023.792 & & \\
\hline
\end{tabular}




\subsection{Accuracy}

Within-Subjects

Factors

\begin{tabular}{|c|c|c|c|c|c|}
\hline \multicolumn{2}{|c|}{ Measure: MEASURE_1 } & \multicolumn{4}{|c|}{ Between-Subjects Factors } \\
\hline \multirow[b]{2}{*}{ time } & \multirow{2}{*}{$\begin{array}{c}\text { Dependent } \\
\text { Variable }\end{array}$} & & & Value Label & $N$ \\
\hline & & \multirow[t]{2}{*}{ Paradigm } & & Serial & 15 \\
\hline \multirow{3}{*}{2} & CPT_accu_pr & & 2.00 & Parallel & 15 \\
\hline & CPT_accu_po & \multirow[t]{2}{*}{ Training } & 0 & Controls & 10 \\
\hline & st & & 1 & Train & 20 \\
\hline
\end{tabular}

Tests of Within-Subjects Contrasts

Measure: MEASURE_1

\begin{tabular}{|ll|r|r|r|r|r|}
\hline Source & time & $\begin{array}{c}\text { Type III Sum } \\
\text { of Squares }\end{array}$ & df & Mean Square & \multicolumn{1}{c|}{ F } & Sig. \\
\hline time & Level 2 vs. Level 1 & 3.151 & 1 & 3.151 & .401 & .532 \\
\hline time * Paradigm & Level 2 vs. Level 1 & .651 & 1 & .651 & .083 & .776 \\
\hline time * Training & Level 2 vs. Level 1 & 1.276 & 1 & 1.276 & .162 & .690 \\
\hline $\begin{array}{l}\text { time * Paradigm * } \\
\text { Training }\end{array}$ & Level 2 vs. Level 1 & 13.776 & 1 & 13.776 & 1.751 & .197 \\
\hline Error(time) & Level 2 vs. Level 1 & 204.531 & 26 & 7.867 & & \\
\hline
\end{tabular}

Tests of Between-Subjects Effects

Measure: MEASURE_1

Transformed Variable: Average

\begin{tabular}{|l|r|r|r|r|r|}
\hline Source & $\begin{array}{c}\text { Type III Sum } \\
\text { of Squares }\end{array}$ & df & Mean Square & \multicolumn{1}{c|}{ F } & Sig. \\
\hline Intercept & 261442.507 & 1 & 261442.507 & 193136.662 & .000 \\
Paradigm & 1.882 & 1 & 1.882 & 1.390 & .249 \\
Training & .059 & 1 & .059 & .043 & .837 \\
Paradigm * Training & .059 & 1 & .059 & .043 & .837 \\
Error & 35.195 & 26 & 1.354 & & \\
\hline
\end{tabular}

\subsubsection{2-way ANOVA for CPT (LEARNING STATUS, TIME)}

\subsection{Reaction time}

\begin{tabular}{|c|c|c|c|c|c|}
\hline \multicolumn{2}{|c|}{$\begin{array}{l}\text { Within-Subjects } \\
\text { Factors }\end{array}$} & \multicolumn{4}{|c|}{ Between-Subjects Factors } \\
\hline \multicolumn{2}{|c|}{ Measure: MEASURE_1 } & & & Value Label & $N$ \\
\hline time & $\begin{array}{c}\text { Dependent } \\
\text { Variable }\end{array}$ & \multirow[t]{2}{*}{ Learner } & .00 & NonLearner & 12 \\
\hline $\begin{array}{l}1 \\
2\end{array}$ & $\begin{array}{l}\text { CPT_rt_pre } \\
\text { CPT_rt_post }\end{array}$ & & 1.00 & Learner & 8 \\
\hline
\end{tabular}

Tests of Within-Subjects Contrasts

Measure: MEASURE_1

\begin{tabular}{|ll|r|r|r|r|r|}
\hline Source & time & $\begin{array}{c}\text { Type III Sum } \\
\text { of Squares }\end{array}$ & \multicolumn{1}{c|}{ df } & Mean Square & \multicolumn{1}{c|}{ F } & \multicolumn{1}{c|}{ Sig. } \\
\hline time & Level 2 vs. Level 1 & 34612.033 & 1 & 34612.033 & 5.386 & .032 \\
\hline time * Learner & Level 2 vs. Level 1 & 11252.033 & 1 & 11252.033 & 1.751 & .202 \\
\hline Error(time) & Level 2 vs. Level 1 & 115680.167 & 18 & 6426.676 & & \\
\hline
\end{tabular}

\subsection{Accuracy}

Within-Subjects Factors

Measure: MEASURE_1

\begin{tabular}{|l|l|}
\hline time & Dependent Variable \\
\hline 1 &
\end{tabular}

\begin{tabular}{|l|l|}
\hline 1 & CPT_accu_pre \\
\hline
\end{tabular}

2 CPT_accu_post
Between-Subjects Factors

\begin{tabular}{|rl|l|r|}
\hline & & Value Label & \multicolumn{1}{|c|}{$\mathrm{N}$} \\
\hline Learner & .00 & NonLearner & 12 \\
& 1.00 & Learner & 8 \\
\hline
\end{tabular}


Tests of Within-Subjects Contrasts

Measure: MEASURE_1
\begin{tabular}{|ll|r|r|r|r|r|}
\hline Source & time & $\begin{array}{c}\text { Type III Sum } \\
\text { of Squares }\end{array}$ & \multicolumn{1}{c|}{ df } & Mean Square & \multicolumn{1}{c|}{ F } & \multicolumn{1}{c|}{ Sig. } \\
\hline time & Level 2 vs. Level 1 & 5.208 & 1 & 5.208 & .635 & .436 \\
\hline time * Learner & Level 2 vs. Level 1 & .833 & 1 & .833 & .102 & .753 \\
\hline Error(time) & Level 2 vs. Level 1 & 147.526 & 18 & 8.196 & & \\
\hline
\end{tabular}

\section{Tests of Between-Subjects Effects}

Measure: MEASURE_1

Transformed Variable: Average

\begin{tabular}{|l|r|r|r|r|r|}
\hline Source & $\begin{array}{c}\text { Type III Sum } \\
\text { of Squares }\end{array}$ & \multicolumn{1}{c|}{ df } & Mean Square & \multicolumn{1}{c|}{ F } & \multicolumn{1}{c|}{ Sig. } \\
\hline Intercept & 188020.833 & 1 & 188020.833 & 134499.353 & .000 \\
Learner & .052 & 1 & .052 & .037 & .849 \\
Error & 25.163 & 18 & 1.398 & & \\
\hline
\end{tabular}

\subsubsection{3-way ANOVA for Flanker (PARADIGM, GROUP, TIME)}

\subsection{Reaction time}

Within-Subjects Factors

Measure: MEASURE_1

\begin{tabular}{|l|l|}
\hline time & \multicolumn{1}{c|}{$\begin{array}{c}\text { Dependent } \\
\text { Variable }\end{array}$} \\
\hline 1 & Flanker_rt_pre \\
2 & Flanker_rt_post \\
\hline
\end{tabular}

Between-Subjects Factors

\begin{tabular}{|ll|l|r|}
\hline & & Value Label & N \\
\hline Paradigm & 1.00 & Serial & 15 \\
& 2.00 & Parallel & 15 \\
Training & 0 & Controls & 10 \\
& 1 & Train & 20 \\
\hline
\end{tabular}

Tests of Within-Subjects Contrasts

Measure: MEASURE_1

\begin{tabular}{|ll|r|r|r|r|r|}
\hline Source & time & $\begin{array}{c}\text { Type III Sum } \\
\text { of Squares }\end{array}$ & \multicolumn{1}{c|}{ df } & Mean Square & \multicolumn{1}{c|}{ F } & \multicolumn{1}{c|}{ Sig. } \\
\hline time & Level 2 vs. Level 1 & 153824.067 & 1 & 153824.067 & 7.616 & .010 \\
\hline time * Paradigm & Level 2 vs. Level 1 & 326.667 & 1 & 326.667 & .016 & .900 \\
\hline time * Training & Level 2 vs. Level 1 & 9576.067 & 1 & 9576.067 & .474 & .497 \\
\hline $\begin{array}{l}\text { time * Paradigm * } \\
\text { Training }\end{array}$ & Level 2 vs. Level 1 & 20609.067 & 1 & 20609.067 & 1.020 & .322 \\
\hline Error(time) & Level 2 vs. Level 1 & 525108.200 & 26 & 20196.469 & & \\
\hline
\end{tabular}

Tests of Between-Subjects Effects

Measure: MEASURE_1

Transformed Variable: Average

\begin{tabular}{|l|r|r|r|r|r|}
\hline Transformed Variable: & \multicolumn{1}{|c|}{ Average } \\
\hline Source & \multicolumn{1}{c|}{$\begin{array}{c}\text { Type III Sum } \\
\text { of Squares }\end{array}$} & \multicolumn{1}{c|}{ df } & Mean Square & \multicolumn{1}{c|}{ F } & \multicolumn{1}{c|}{ Sig. } \\
\hline Intercept & 32432612.82 & 1 & 32432612.82 & 1190.255 & .000 \\
Paradigm & 13500.000 & 1 & 13500.000 & .495 & .488 \\
Training & 109.350 & 1 & 109.350 & .004 & .950 \\
Paradigm * Training & 29837.400 & 1 & 29837.400 & 1.095 & .305 \\
Error & 708459.650 & 26 & 27248.448 & & \\
\hline
\end{tabular}




\subsection{Accuracy}

\begin{tabular}{|c|c|c|c|c|c|}
\hline \multirow{2}{*}{\multicolumn{2}{|c|}{ Within-Subjects Factors }} & \multicolumn{4}{|c|}{ Between-Subjects Factors } \\
\hline & & & & & \multirow{3}{*}{$\begin{array}{l}\mathrm{N} \\
15 \\
15\end{array}$} \\
\hline \multicolumn{2}{|c|}{ Measure: MEASURE_1 } & \multirow[t]{2}{*}{ Paradigm } & \multirow{2}{*}{$\begin{array}{l}1.00 \\
2.00\end{array}$} & \multirow{4}{*}{$\begin{array}{l}\text { Serial } \\
\text { Parallel } \\
\text { Controls } \\
\text { Train }\end{array}$} & \\
\hline time & Dependent Variable & & & & \\
\hline 1 & Flanker_accu_pre & Training & 0 & & 10 \\
\hline 2 & Flanker_accu_post & & 1 & & 20 \\
\hline
\end{tabular}

Tests of Within-Subjects Contrasts

Measure: MEASURE_1

\begin{tabular}{|ll|r|r|r|r|r|}
\hline Source & time & $\begin{array}{c}\text { Type III Sum } \\
\text { of Squares }\end{array}$ & \multicolumn{1}{c|}{$\mathrm{df}$} & \multicolumn{1}{c|}{ Mean Square } & \multicolumn{1}{c|}{ F } & \multicolumn{1}{c|}{ Sig. } \\
\hline time & Level 2 vs. Level 1 & 89.695 & 1 & 89.695 & 3.167 & .087 \\
\hline time * Paradigm & Level 2 vs. Level 1 & .186 & 1 & .186 & .007 & .936 \\
\hline time * Training & Level 2 vs. Level 1 & 47.384 & 1 & 47.384 & 1.673 & .207 \\
\hline $\begin{array}{l}\text { time * Paradigm * } \\
\text { Training }\end{array}$ & Level 2 vs. Level 1 & .186 & 1 & .186 & .007 & .936 \\
\hline Error(time) & Level 2 vs. Level 1 & 736.369 & 26 & 28.322 & & \\
\hline
\end{tabular}

\subsubsection{2-way ANOVA for Flanker (LEARNING STATUS, TIME)}

\subsection{Reaction time}

\section{Within-Subjects Factors}

Measure: MEASURE_1
\begin{tabular}{|l|l|}
\hline time & $\begin{array}{c}\text { Dependent } \\
\text { Variable }\end{array}$ \\
\hline 1 & Flanker_rt_pre \\
2 & Flanker_rt_post \\
\hline
\end{tabular}

Between-Subjects Factors

\begin{tabular}{|rl|l|r|}
\hline & & Value Label & \multicolumn{1}{|c|}{$\mathrm{N}$} \\
\hline Learner & .00 & NonLearner & 12 \\
& 1.00 & Learner & 8 \\
\hline
\end{tabular}

\section{Tests of Within-Subjects Contrasts}

Measure: MEASURE_1

\begin{tabular}{|ll|r|r|r|r|r|}
\hline Source & time & $\begin{array}{c}\text { Type III Sum } \\
\text { of Squares }\end{array}$ & df & Mean Square & F & Sig. \\
\hline time & Level 2 vs. Level 1 & 42225.008 & 1 & 42225.008 & 2.039 & .170 \\
\hline time * Learner & Level 2 vs. Level 1 & 49005.208 & 1 & 49005.208 & 2.367 & .141 \\
\hline Error(time) & Level 2 vs. Level 1 & 372684.792 & 18 & 20704.711 & & \\
\hline
\end{tabular}

\section{Tests of Between-Subjects Effects}

Measure: MEASURE_1

Transformed Variable: Average

\begin{tabular}{|l|c|r|r|r|r|}
\hline Source & \multicolumn{1}{|c|}{$\begin{array}{c}\text { Type III Sum } \\
\text { of Squares }\end{array}$} & df & Mean Square & \multicolumn{1}{c|}{ F } & \multicolumn{1}{c|}{ Sig. } \\
\hline Intercept & 22645706.42 & 1 & 22645706.42 & 1037.097 & .000 \\
Learner & 104695.669 & 1 & 104695.669 & 4.795 & .042 \\
Error & 393042.031 & 18 & 21835.668 & & \\
\hline
\end{tabular}




\subsection{Accuracy}

Within-Subjects Factors

Measure: MEASURE_1 Between-Subjects Factors
\begin{tabular}{|l|l|l|l|l|r|}
\hline time & Dependent Variable \\
\hline 1 & Flanker_accu_pre \\
2 & Flanker_accu_post \\
\cline { 2 - 5 }
\end{tabular}

Tests of Within-Subjects Contrasts

Measure: MEASURE_1

\begin{tabular}{|ll|r|r|r|r|r|}
\hline Source & time & $\begin{array}{c}\text { Type III Sum } \\
\text { of Squares }\end{array}$ & \multicolumn{1}{c|}{ df } & Mean Square & \multicolumn{1}{c|}{ F } & Sig. \\
\hline time & Level 2 vs. Level 1 & 7.525 & 1 & 7.525 & .239 & .630 \\
\hline time * Learner & Level 2 vs. Level 1 & 7.505 & 1 & 7.505 & .239 & .631 \\
\hline Error(time) & Level 2 vs. Level 1 & 565.564 & 18 & 31.420 & & \\
\hline
\end{tabular}

\section{Tests of Between-Subjects Effects}

Measure: MEASURE_1

Transformed Variable: Average

\begin{tabular}{|l|r|r|r|r|r|}
\hline Source & $\begin{array}{c}\text { Type III Sum } \\
\text { of Squares }\end{array}$ & df & Mean Square & \multicolumn{1}{|c|}{ F } & \multicolumn{1}{c|}{ Sig. } \\
\hline Intercept & 170878.797 & 1 & 170878.797 & 11209.296 & .000 \\
Learner & .023 & 1 & .023 & .002 & .969 \\
Error & 274.399 & 18 & 15.244 & & \\
\hline
\end{tabular}

\subsubsection{3-way ANOVA for Flanker (CONGRUENCY, LEARNING STATUS, TIME)}

\subsection{Reaction time}

Within-Subjects Factors

Measure: MEASURE_1

Measure: MEASURE_1
\begin{tabular}{|ll|l|}
\hline TIME & CONGRUENCE & \multicolumn{1}{|c|}{ Dependent Variable } \\
\hline 1 & 1 & Flanker_rt_cong_pre \\
& 2 & Flanker_rt_incong_pre \\
\hline 2 & 1 & Flanker_rt_cong_post \\
& 2 & Flanker_rt_incong_post \\
\hline
\end{tabular}

Between-Subjects Factors

\begin{tabular}{|rl|l|r|}
\hline & & Value Label & \multicolumn{1}{|c|}{$\mathrm{N}$} \\
\hline Learner & .00 & NonLearner & 12 \\
& 1.00 & Learner & 8 \\
\hline
\end{tabular}

Tests of Within-Subjects Contrasts

Measure: MEASURE_1

\begin{tabular}{|c|c|c|c|c|c|c|c|}
\hline Source & TIME & CONGRUENCE & $\begin{array}{c}\text { Type III Sum } \\
\text { of Squares }\end{array}$ & df & Mean Square & $\mathrm{F}$ & Sig. \\
\hline TIME & Level 2 vs. Level 1 & & 41888.033 & 1 & 41888.033 & 2.024 & .172 \\
\hline TIME * Learner & Level 2 vs. Level 1 & & 48884.033 & 1 & 48884.033 & 2.362 & .142 \\
\hline Error(TIME) & Level 2 vs. Level 1 & & 372491.667 & 18 & 20693.981 & & \\
\hline CONGRUENCE & & Level 2 vs. Level 1 & 71053.333 & 1 & 71053.333 & 19.072 & .000 \\
\hline CONGRUENCE * Learner & & Level 2 vs. Level 1 & 2.133 & 1 & 2.133 & .001 & .981 \\
\hline Error(CONGRUENCE) & & Level 2 vs. Level 1 & 67061.167 & 18 & 3725.620 & & \\
\hline TIME * CONGRUENCE & Level 2 vs. Level 1 & Level 2 vs. Level 1 & 71150.700 & 1 & 71150.700 & 7.453 & .014 \\
\hline TIME * CONGRUENCE * Learner & Level 2 vs. Level 1 & Level 2 vs. Level 1 & 86.700 & 1 & 86.700 & .009 & .925 \\
\hline Error(TIME*CONGRUENCE) & Level 2 vs. Level 1 & Level 2 vs. Level 1 & 171829.500 & 18 & 9546.083 & & \\
\hline
\end{tabular}




\section{Tests of Between-Subjects Effects}

Measure: MEASURE_1

Transformed Variable: Average

\begin{tabular}{|l|c|r|r|r|r|}
\hline Source & $\begin{array}{c}\text { Type III Sum } \\
\text { of Squares }\end{array}$ & df & Mean Square & \multicolumn{1}{c|}{ F } & \multicolumn{1}{c|}{ Sig. } \\
\hline Intercept & 22647444.10 & 1 & 22647444.10 & 1037.808 & .000 \\
Learner & 104459.502 & 1 & 104459.502 & 4.787 & .042 \\
Error & 392803.010 & 18 & 21822.389 & & \\
\hline
\end{tabular}

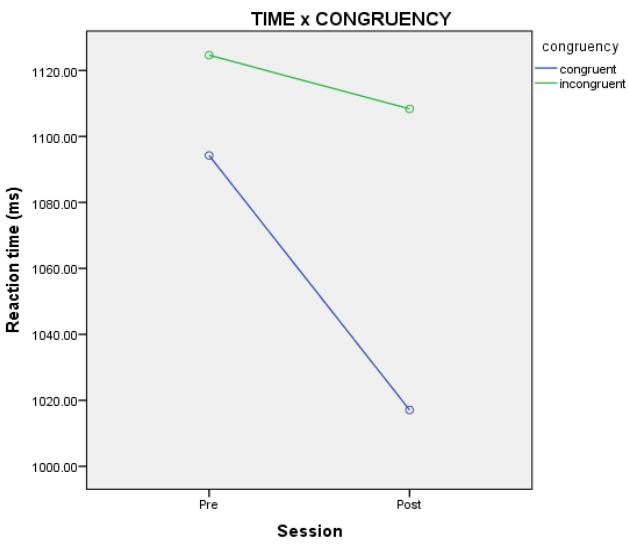

\subsection{Accuracy}

Within-Subjects Factors

Measure: MEASURE_1

\begin{tabular}{|ll|l|}
\hline TIME & CONGRUENCE & \multicolumn{1}{|c|}{ Dependent Variable } \\
\hline 1 & 1 & Flanker_accu_cong_pre \\
& 2 & Flanker_accu_incong_pre \\
\hline 2 & 1 & Flanker_accu_cong_post \\
& 2 & Flanker_accu_incong_post \\
\hline
\end{tabular}

Between-Subjects Factors
\begin{tabular}{|rl|l|r|}
\hline & & Value Label & \multicolumn{1}{|c|}{ N } \\
\hline Learner & .00 & NonLearner & 12 \\
& 1.00 & Learner & 8 \\
\hline
\end{tabular}

Tests of Within-Subjects Contrasts

Measure: MEASURE_1

\begin{tabular}{|c|c|c|c|c|c|c|c|}
\hline Source & TIME & CONGRUENCE & $\begin{array}{c}\text { Type III Sum } \\
\text { of Squares }\end{array}$ & $\mathrm{df}$ & Mean Square & $\mathrm{F}$ & Sig. \\
\hline TIME & Level 2 vs. Level 1 & & 7.528 & 1 & 7.528 & .240 & .630 \\
\hline TIME * Learner & Level 2 vs. Level 1 & & 7.518 & 1 & 7.518 & .239 & .631 \\
\hline Error(TIME) & Level 2 vs. Level 1 & & 565.485 & 18 & 31.416 & & \\
\hline CONGRUENCE & & Level 2 vs. Level 1 & 204.637 & 1 & 204.637 & 10.109 & .005 \\
\hline CONGRUENCE * Learner & & Level 2 vs. Level 1 & 33.502 & 1 & 33.502 & 1.655 & .215 \\
\hline Error(CONGRUENCE) & & Level 2 vs. Level 1 & 364.361 & 18 & 20.242 & & \\
\hline TIME * CONGRUENCE & Level 2 vs. Level 1 & Level 2 vs. Level 1 & .373 & 1 & .373 & .006 & .940 \\
\hline TIME * CONGRUENCE * Learner & Level 2 vs. Level 1 & Level 2 vs. Level 1 & 18.182 & 1 & 18.182 & .288 & .598 \\
\hline Error(TIME*CONGRUENCE) & Level 2 vs. Level 1 & Level 2 vs. Level 1 & 1135.232 & 18 & 63.068 & & \\
\hline
\end{tabular}

Tests of Between-Subjects Effects

Measure: MEASURE_1

Transformed Variable: Average

\begin{tabular}{|l|r|r|r|r|r|}
\hline Source & $\begin{array}{c}\text { Type III Sum } \\
\text { of Squares }\end{array}$ & df & Mean Square & \multicolumn{1}{c|}{ F } & Sig. \\
\hline Intercept & 170883.136 & 1 & 170883.136 & 11205.824 & .000 \\
Learner & .023 & 1 & .023 & .002 & .969 \\
Error & 274.491 & 18 & 15.249 & & \\
\hline
\end{tabular}




\title{
Curriculum Vitae
}

\author{
WAN ILMA DEWIPUTRI \\ B.S c (Hons.)
}

Im Hassel 38

37077 Göttingen

Germany

wdewipu@gwdg.de

\section{PERSONAL DETAILS}

Date of birth: 20 December 1985

Place of birth: Georgetown, Penang, Malaysia

Nationality: Malaysian

\section{E D U C A T I O N}

Georg-August-Universität Göttingen, Göttingen, Germany, 2010 - present

Doctoral candidate in the International Max Planck Research School for Neuroscience

University of Adelaide, Adelaide, Australia, 2005 - 2008

Bachelor of Health Science (First Class Honours)

International Education Center, Shah Alam, Malaysia, 2003 - 2004

South Australian Matriculation

Kolej Tunku Kurshiah, Seremban, Malaysia, 1998 - 2002

\section{RESEARCH EXPERIENCE}

Biomedizinische NMR Forschungs GmbH | Max Planck Institute of Biophysical Chemsitry, Goettingen, 2011-2014

Doctoral student

"Real-time fMRI neurofeedback in cognition"

Lab Rotation student

Advisor: Prof. Dr. Jens Frahm, Dr. Renate Schweizer, Dr. Tibor Auer

Inner Ear Lab | Dept. Otorhynolaryngology, Universitätsmedizin Göttingen, 2011

Lab rotation student

"Prepulse inhibition of the acoustic startle response: Are natural sounds effective stimuli?"

Advisor: Dr. Nicola Strenzke

Invertebrate Neuropharmacology Lab | Georg-August Universität Göttingen, 2011

Lab rotation student

"Neuronal projections from the suboesophageal ganglion into the corpora allata of Locusta migratoria"

Advisor: Prof. Dr. Ralf Heinrich, Dr. Andrea Wirmer

Women's and Children's Health Research Institute | Adelaide, 2008

Honours year research student

"Expression of retinol-binding proteins in calvarial suture cells from patients with

craniosynostosis" 
Advisor: Prof. Dr. Barry Powell, Dr. Peter Anderson, Dr. Prem Dwivedi

Molecular Plant Breeding Cooperative Research Center | Adelaide, 2007

Summer program research student

"Population genetics of barley pathogens Rhyncosporium secalis"

Advisor: Dr. Felicity Keiper

\section{TEACHING EXPERIENCE}

Class tutor I International Max Planck Research School, Göttingen, 2014 and 2013 Tutoring M.Sc Neuroscience students on fMRI

Lab rotation supervisor | Biomedizinische NMR Forschungs GmbH, Göttingen, 2013 Two-month supervision of two M.Sc Neuroscience students (Sarah Lam, Sneha Shashidhara)

\section{COURSES AND WORKSHOPS}

Scientific Writing for The Public | Georg-August Universität Göttingen, 2013

Advanced Neuroimaging Training Program | University of California Los Angeles, USA, 2012

Tübingen Spring School: "Methods to study brain in action" | University of Tübingen, Germany, 2012

Various short GGNB Courses: MATLAB, Psychophysics, Python, Adobe Illustrator, Voice training | Georg-August Universität Göttingen, 2011-2014

\section{CONFERENCES AND CONTRIBUTIONS}

$2^{\text {th }}$ Organization of Human Brain Mapping Conference | Hamburg, Germany, 2014 Poster: "Uncoupling task and feedback processing is promising in fMRI neurofeedback of the anterior mid-cingulate cortex"

$1^{\text {th }}$ Organization of Human Brain Mapping Conference | Seattle, USA, 2014 Poster: “Uncoupling task and feedback processing during cognitive fMRI neurofeedback training"

$9^{\text {th }}$ International Conference on Cognitive Science | Kuching, Malaysia, 2013 Poster: "Uncoupling task and feedback processing during cognitive fMRI neurofeedback training"

$5^{\text {th }}$ NEURIZONS Conference | Göttingen, Germany, 2014

Organizer, Graphic designer.

1 $^{\text {st }}$ Swiss rt-fMRI Neurofeedback Conference | Zurich, Switzerland, 2012 


\section{PUBLICATIONS}

Dewiputri W.I., Auer T. "Functional MRI in neurofeedback: implementations and applications". Malays J Med Sci. 2013. 20(5)

In preparation:

Dewiputri W.I., Auer T, Schweizer R, Frahm J. "Functional localization of the anterior mid-cingulate cortex for real-time fMRI neurofeedback"

Dewiputri W.I., Auer T, Schweizer R, Frahm J. "Uncoupling task and feedback processing is promising in fMRI neurofeedback of the anterior mid-cingulate cortex"

\section{SCHOLARSHIPS AND AWARDS}

PhD scholarship | Malaysian Ministry of Higher Education, 2010 - 2014

Honours Scholarship | Women's and Children's Health Research Institute, 2008

Merdeka Awards | Australia-Malaysia Business Council SA Inc., 2008

Summer research scholarship | Molecular Plant Breeding Cooperative Research Center, 2007

Bachelor scholarship | Malaysian Public Service Department, 2004 - 2008 